\title{
ORBIT PARAMETRIZATIONS FOR K3 SURFACES
}

\author{
MANJUL BHARGAVA ${ }^{1}$, WEI HO ${ }^{2}$ and ABHINAV KUMAR ${ }^{3,4}$ \\ ${ }^{1}$ Department of Mathematics, Princeton University, Princeton, NJ 08544, USA; \\ email: bhargava@math.princeton.edu \\ ${ }^{2}$ Department of Mathematics, University of Michigan, Ann Arbor, MI 48109, USA; \\ email: weiho@umich.edu \\ ${ }^{3}$ Department of Mathematics, Massachusetts Institute of Technology, \\ Cambridge, MA 02139, USA \\ ${ }^{4}$ Department of Mathematics, Stony Brook University, Stony Brook, NY 11794, USA; \\ email: thenav@gmail.com
}

Received 4 August 2014; accepted 4 May 2016

\begin{abstract}
We study moduli spaces of lattice-polarized K3 surfaces in terms of orbits of representations of algebraic groups. In particular, over an algebraically closed field of characteristic 0, we show that in many cases, the nondegenerate orbits of a representation are in bijection with $\mathrm{K} 3$ surfaces (up to suitable equivalence) whose Néron-Severi lattice contains a given lattice. An immediate consequence is that the corresponding moduli spaces of these lattice-polarized K3 surfaces are all unirational. Our constructions also produce many fixed-point-free automorphisms of positive entropy on $\mathrm{K} 3$ surfaces in various families associated to these representations, giving a natural extension of recent work of Oguiso.
\end{abstract}

2010 Mathematics Subject Classification: 14J28, 14J10, 14J50, 11Exx

\section{Contents}

1 Introduction

2 Preliminaries $\quad 12$

2.1 Lattices . . . . . . . . . . . . . . . . . . . . . . . . . . . . . . 12

$2.2 \mathrm{~K} 3$ surfaces . . . . . . . . . . . . . . . 13

2.3 Notation and conventions . . . . . . . . . . . . 15

(C) The Author(s) 2016. This is an Open Access article, distributed under the terms of the Creative Commons Attribution licence (http://creativecommons.org/licenses/by/4.0/), which permits unrestricted re-use, distribution, and reproduction in any medium, provided the original work is properly cited. 
3 Some classical moduli spaces for K3 surfaces with low Picard number 15

3.1 Elliptic surfaces with section . . . . . . . . . . . 16

3.2 Double covers of $\mathbb{P}^{1} \times \mathbb{P}^{1} \ldots \ldots \ldots \ldots \ldots$

3.3 Hypersurfaces of bidegree $(2,3)$ in $\mathbb{P}^{1} \times \mathbb{P}^{2} \ldots \ldots \ldots$

3.4 Complete intersection of bidegree $(1,1)$ and $(2,2)$ hypersurfaces in $\mathbb{P}^{2} \times \mathbb{P}^{2} \ldots \ldots \ldots \ldots \ldots \ldots$

3.5 Complete intersection of bidegree $(1,2)$ and $(2,1)$ hypersurfaces in $\mathbb{P}^{2} \times \mathbb{P}^{2} \ldots \ldots \ldots \ldots \ldots \ldots$. . . . . . . . . . . . . . . .

3.6 Hypersurfaces of tridegree $(2,2,2)$ in $\mathbb{P}^{1} \times \mathbb{P}^{1} \times \mathbb{P}^{1} \ldots \ldots . .17$

4 Rubik's revenge: $4 \otimes 4 \otimes 4 \quad 17$

4.1 Construction of $\mathrm{K} 3$ surfaces $\ldots \ldots \ldots \ldots$

4.2 Néron-Severi lattice . . . . . . . . . . . . . . . . . . . . 19

4.3 Moduli problem . . . . . . . . . . . . . . . . 21

4.4 Automorphisms ..................... 24

5 Doubly symmetric Rubik's revenge: $4 \otimes \mathrm{Sym}^{2}(4) \quad \mathbf{2 5}$

5.1 Néron-Severi lattice . . . . . . . . . . . . . . 26

5.2 Moduli problem . . . . . . . . . . . . . . . . . . 27

5.3 Automorphisms . . . . . . . . . . . . . . . . . 29

6 Triply symmetric Rubik's revenge: $\operatorname{Sym}^{3}(4) \quad 30$

6.1 Néron-Severi lattice . . . . . . . . . . . . . . 31

6.2 Moduli problem . . . . . . . . . . . . . . . . . . 31

6.3 Automorphisms . . . . . . . . . . . . . . 32

7 Penteracts (or 5-cubes): $2 \otimes 2 \otimes 2 \otimes 2 \otimes 2 \quad 32$

7.1 Constructions of $\mathrm{K} 3$ surfaces . . . . . . . . . . . . . 33

7.2 Néron-Severi lattice . . . . . . . . . . . . . . . . 34

7.3 Reverse construction . . . . . . . . . . . . . . 36

7.4 Automorphisms ................... 38

8 Doubly symmetric penteracts: $2 \otimes 2 \otimes 2 \otimes \operatorname{Sym}^{2}(2) \quad 41$

8.1 Néron-Severi lattice . . . . . . . . . . . . . . . . 42

8.2 Moduli problem . . . . . . . . . . . . . . . . . . . . 44

8.3 Automorphisms . . . . . . . . . . . . . . . . . . 44

9 Triply symmetric penteracts: $2 \otimes 2 \otimes \operatorname{Sym}^{3}(2) \quad 45$

9.1 Néron-Severi lattice . . . . . . . . . . . . . . . . . . . 46

9.2 Moduli problem . . . . . . . . . . . . . . . . . . . 48

9.3 Automorphisms . . . . . . . . . . . . . . . . . . 48 
10 Doubly doubly symmetric penteracts: $2 \otimes \operatorname{Sym}^{2}(2) \otimes \operatorname{Sym}^{2}(2) \quad 49$

10.1 Néron-Severi lattice and moduli problem . . . . . . . . . 50

10.2 Automorphisms .................. 53

11 Doubly triply symmetric penteracts: $\operatorname{Sym}^{2}(2) \otimes \operatorname{Sym}^{3}(2) \quad 54$

11.1 Néron-Severi lattice and moduli problem . . . . . . . . . 55

11.2 Automorphisms ................... 58

12 Quadruply symmetric penteracts: $2 \otimes \operatorname{Sym}^{4}(2) \quad 58$

12.1 Néron-Severi lattice and moduli problem . . . . . . . . . . 59

12.2 Automorphisms .................. 62

13 Quintuply symmetric penteracts: $\operatorname{Sym}^{5}(2) \quad 62$

13.1 Néron-Severi lattice and moduli problem . . . . . . . . . . 63

13.2 Automorphisms .................. . 66

$142 \otimes 2 \otimes 2 \otimes 4 \quad 66$

14.1 Construction of $\mathrm{K} 3$ surfaces . . . . . . . . . . . . . 67

14.2 Singularities and exceptional divisors . . . . . . . . . . . 68

14.3 Néron-Severi lattice . . . . . . . . . . . . . . . . 69

14.4 Reverse map .................... 72

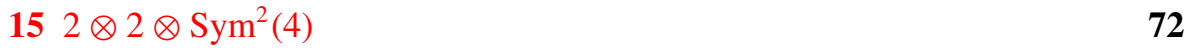

15.1 Construction of $\mathrm{K} 3$ surfaces . . . . . . . . . . . . 73

15.2 Néron-Severi lattice ................. . . 74

15.3 Moduli problem ................... 74

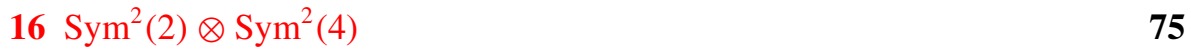

17 Applications and connections $\quad 76$

17.1 Definition of hyperdeterminant . . . . . . . . . . . . 77

17.2 Interpretations in terms of fixed-point-free automorphisms . . . 77

17.3 Fixed-point-free automorphisms of finite order . . . . . . . . . 79

17.4 Fixed-point-free automorphisms of positive entropy . . . . . . . . 80

$\begin{array}{lr}\text { References } & 83\end{array}$

\section{Introduction}

An important classical problem is that of classifying the orbits of a representation, over a field or over a ring, in terms of suitable algebraic or 
geometric objects over that field or ring; conversely, one may wish to construct representations whose orbits parametrize given algebraic or geometric objects of interest.

In recent years, there have been a number of arithmetic applications of such 'orbit parametrizations' for geometric objects of dimension 0 and of dimension 1 . For example, for extensions of a field, or ring extensions and ideal classes in those extensions, such parametrizations have been studied in numerous papers, including [7-9, 11, 29, 74-76]; these descriptions of the moduli spaces have been used in an essential way in many applications (see, for example, $[10,12,19,28$, $69,73]$ ). In fact, many of the cleanest and most useful such bijections between rings/ideal classes and orbits of representations have arisen in cases where the representation is prehomogeneous, that is, where the ring of (relative) invariants is a polynomial ring with one generator.

In the case of curves, recent work on orbit parametrizations in cases of arithmetic interest over a general base field include [16] for genus one curves and $[15,70]$ for various types of higher genus curves. Numerous examples have also been previously considered by algebraic geometers, even classically, often giving descriptions of the coarse moduli space as a GIT quotient. As before, many of the most arithmetically useful bijections between data relating to algebraic curves and orbits of representations have arisen in cases where the representation has somewhat simple invariant theory-in particular, when the representation is coregular, meaning that the ring of relative invariants is a polynomial ring. In these cases, the coarse moduli space of the geometric data is thus (an open subvariety of) a weighted projective space. In conjunction with geometry-ofnumbers and other analytic counting and sieve arguments, such representations have seen applications in bounding average ranks in families of elliptic curves over $\mathbb{Q}$ (see, for example, $[17,18]$ ) and showing that many curves in families of higher genus have few rational points (see $[13,15,62,67]$ ).

A natural next step is to determine representations whose orbits parametrize geometric data of interest associated to algebraic surfaces. K3 surfaces form a rich class of surfaces that naturally lend themselves to such a study, and in fact, there has already been significant work in this direction (albeit usually over algebraically closed fields). For example, it is classically known that a general polarized K3 surface of genus $g=3$, 4, or 5 may be described as a complete intersection in projective space $\mathbb{P}^{g}$, and such descriptions may be easily translated into the language of orbits of a representation of an algebraic group. For polarized K3 surfaces of higher genus, Mukai and others have also described them in several cases as complete intersections in homogeneous spaces (see, for example, [53-55]). A sample of these results for polarizations of small degree appears in Table 2. 
Table 1. Representations $V$ whose $G$-orbits parametrize data related to K3 surfaces. The group $G^{\text {ss }}$ is a semisimple algebraic group with a map to the group $G$, whose kernel is finite and cokernel is solvable. Here $\mathfrak{O}_{D}$ denotes the lattice underlying the quadratic ring of discriminant $D$ with the quadratic form being twice the norm form. Root lattices are normalized to be negative definite, and $U \cong \mathfrak{O}_{1}$ denotes the hyperbolic plane.

\begin{tabular}{|c|c|c|c|c|c|}
\hline No. & Group $G^{\text {ss }}$ & Representation $V$ & Rank & Generic NS & Section \\
\hline 1 & $\mathrm{SL}_{2}$ & $\operatorname{Sym}^{8}(2) \oplus \operatorname{Sym}^{12}(2)$ & 2 & $\mathfrak{D}_{1}=U$ & 3.1 \\
\hline 2 & $\mathrm{SL}_{2}^{2}$ & $\operatorname{Sym}^{4}(2) \otimes \operatorname{Sym}^{4}(2)$ & 2 & $U(2)$ & 3.2 \\
\hline 3 & $\mathrm{SL}_{2} \times \mathrm{SL}_{3}$ & $\operatorname{Sym}^{2}(2) \otimes \operatorname{Sym}^{3}(3)$ & 2 & $\mathfrak{O}_{9}$ & 3.3 \\
\hline 4 & $\mathrm{SL}_{3}^{2}$ & $3 \otimes 3 \oplus \operatorname{Sym}^{2}(3) \otimes \operatorname{Sym}^{2}(3)$ & 2 & $\mathfrak{O}_{12}$ & 3.4 \\
\hline 5 & $\mathrm{SL}_{2}^{2} \times \mathrm{SL}_{4}$ & $2 \otimes 2 \otimes \operatorname{Sym}^{2}(4)$ & 2 & $\mathfrak{O}_{4}(2)$ & 15 \\
\hline 6 & $\mathrm{SL}_{4}^{3}$ & $4 \otimes 4 \otimes 4$ & 2 & $\mathfrak{O}_{5}(2)$ & 4 \\
\hline 7 & $\mathrm{SL}_{3}^{2}$ & $3 \otimes \operatorname{Sym}^{2}(3) \oplus \operatorname{Sym}^{2}(3) \otimes 3$ & 2 & $\mathfrak{D}_{21}$ & 3.5 \\
\hline 8 & $\mathrm{SL}_{2}^{3}$ & $\operatorname{Sym}^{2}(2) \otimes \operatorname{Sym}^{2}(2) \otimes \operatorname{Sym}^{2}(2)$ & 3 & $U(2) \oplus\langle-4\rangle$ & 3.6 \\
\hline 9 & $\mathrm{SL}_{2}^{5}$ & $2 \otimes 2 \otimes 2 \otimes 2 \otimes 2$ & 4 & $U(2) \oplus A_{2}(2)$ & 7 \\
\hline 10 & $\mathrm{SL}_{2} \times \mathrm{SL}_{4}$ & $\operatorname{Sym}^{2}(2) \otimes \operatorname{Sym}^{2}(4)$ & 9 & $U \oplus E_{7}(2)$ & 16 \\
\hline 11 & $\mathrm{SL}_{2}^{4}$ & $2 \otimes 2 \otimes 2 \otimes \operatorname{Sym}^{2}(2)$ & 9 & $U \oplus E_{7}(2)$ & 8 \\
\hline 12 & $\mathrm{SL}_{4}^{2}$ & $4 \otimes \operatorname{Sym}^{2}(4)$ & 11 & $U \oplus E_{8}(2) \oplus\langle-4\rangle$ & 5 \\
\hline 13 & $\mathrm{SL}_{2}^{3}$ & $2 \otimes \operatorname{Sym}^{2}(2) \otimes \operatorname{Sym}^{2}(2)$ & 12 & $U(4) \oplus E_{8} \oplus\langle-4\rangle^{\oplus 2}$ & 10 \\
\hline 14 & $\mathrm{SL}_{2}^{3} \times \mathrm{SL}_{4}$ & $2 \otimes 2 \otimes 2 \otimes 4$ & 13 & $\langle 4\rangle \oplus\langle-2\rangle^{\oplus 4} \oplus D_{4}^{\oplus 2}$ & 14 \\
\hline 15 & $\mathrm{SL}_{2}^{3}$ & $2 \otimes 2 \otimes \operatorname{Sym}^{3}(2)$ & 14 & $U(2) \oplus A_{2}^{\oplus 3} \oplus E_{6}$ & 9 \\
\hline 16 & $\mathrm{SL}_{2}^{2}$ & $\operatorname{Sym}^{2}(2) \otimes \operatorname{Sym}^{3}(2)$ & 15 & $U \oplus A_{2}^{\oplus 2} \oplus E_{6} \oplus A_{3}$ & 11 \\
\hline 17 & $\mathrm{SL}_{4}$ & $\operatorname{Sym}^{3}(4)$ & 16 & $U(2) \oplus A_{2} \oplus D_{12}$ & 6 \\
\hline 18 & $\mathrm{SL}_{2}^{2}$ & $2 \otimes \operatorname{Sym}^{4}(2)$ & 17 & $U \oplus\langle-8\rangle \oplus D_{12} \oplus A_{2}$ & 12 \\
\hline 19 & $\mathrm{SL}_{2}$ & $\operatorname{Sym}^{5}(2)$ & 18 & $U \oplus A_{4} \oplus D_{12}$ & 13 \\
\hline
\end{tabular}

The purpose of this paper is to generalize these ideas to study moduli spaces of K3 surfaces with possibly multiple line bundles, namely lattice-polarized K3 surfaces, in terms of the orbits of suitable representations. More precisely, the classes of line bundles in the Picard group of a K3 surface $X$ naturally form a lattice, with the symmetric bilinear pairing being the intersection pairing on divisors of the surface; this is called the Néron-Severi lattice of $X$. There is a coarse moduli space $\mathcal{M}_{\Lambda}$ of K3 surfaces whose Néron-Severi lattice contains a fixed lattice $\Lambda$. (We will actually work with a slight modification of this moduli space; see Section 2.2.) It is a quasiprojective variety, but in general it is very difficult to explicitly describe it by equations or to understand its geometry, especially if $\operatorname{rank}(\Lambda)>1$. We show that there are at least 19 representations of algebraic groups whose orbits naturally parametrize such lattice-polarized $\mathrm{K} 3$ surfaces. We list them in Table 1. Some of these orbit parametrizations are classical, but most of the higher rank cases appear to be new. 
Just as $2 \times 2 \times 2$ cubical matrices played a key role in the understanding of many prehomogeneous representations [7], and just as $3 \times 3 \times 3$ and $2 \times 2 \times$ $2 \times 2$ matrices played a key role in the understanding of coregular representations associated to genus one curves [16], we find that $4 \times 4 \times 4$ and $2 \times 2 \times 2 \times$ $2 \times 2$ matrices appear as fundamental cases for our study of K3 surfaces. We refer to these cases as the 'Rubik's revenge' and 'penteract' cases, respectively. We also study orbits on symmetrized versions of these spaces, which turn out to correspond to moduli spaces of K3 surfaces of higher rank. For example, we show that $\mathrm{GL}_{2}$-orbits on the space of quintuply symmetric penteracts-that is, binary quintic forms - correspond to elements of a certain family of K3 surfaces having rank at least 18.

We now state our main theorem more precisely. Given a K3 surface defined over a field $F$ having algebraic closure $\bar{F}$, let $\mathrm{NS}(X)$ denote the Néron-Severi group of $X$, that is, the group of divisors on $X$ over $F$ modulo algebraic equivalence. Let $\overline{\mathrm{NS}}(X)$ be $\mathrm{NS}\left(X_{\bar{F}}\right)$. (When $F$ is algebraically closed, we have $\mathrm{NS}(X)=\overline{\mathrm{NS}}(X)$.) Then we define a lattice-polarized K3 surface over $F$ as follows.

DEFINITION 1.1. Let $\Lambda$ be an even nondegenerate lattice with signature $(1, s)$ with a choice of basis, and let $\Sigma$ be a saturated sublattice of $\Lambda$. Then we say that a K3 surface $X$ over $F$ is lattice-polarized by $(\Lambda, \Sigma)$ if there exists a primitive lattice embedding $\phi: \Lambda \rightarrow \overline{\mathrm{NS}}(X)$ such that $\phi(\Sigma)$ is fixed pointwise by the action of $\operatorname{Gal}(\bar{F} / F)$ and the image under $\phi$ of a certain subset $C(\Lambda)$ of $\Lambda$ contains an ample divisor class of $X$.

We now define the subset $C(\Lambda)$. Let $Z(\Lambda):=\{z \in \Lambda:\langle z, z\rangle=-2\}$ be the set of roots of $\Lambda$. Fix a partitioning of $Z(\Lambda)$ into two subsets $Z(\Lambda)^{+}$and $Z(\Lambda)^{-}$, where $Z(\Lambda)^{-}=\left\{-z: z \in Z(\Lambda)^{+}\right\}$and each is closed under positive finite sums; also fix a connected component $V$ of the cone $\{z \in \Lambda \otimes \mathbb{R}:\langle z, z\rangle>0\}$. We then let $C(\Lambda)$ be the subset of $V \cap \Lambda$ consisting of elements that pair positively with all $z \in Z(\Lambda)^{+}$; this is the intersection of $\Lambda$ with the Weyl chamber defined by the positive roots.

Let $\mathcal{M}_{\Lambda, \Sigma}$ denote the moduli space of such pairs $(X, \phi)$, where $X$ is a $\mathrm{K} 3$ surface lattice-polarized by $(\Lambda, \Sigma)$ and $\phi: \Lambda \rightarrow \overline{\mathrm{NS}}(X)$ is a primitive lattice embedding, modulo equivalence; two pairs $(X, \phi)$ and $(Y, \psi)$ are equivalent if there exists an isomorphism $f: Y \rightarrow X$ and an isometry $g: \Lambda \rightarrow \Lambda$ fixing $\Sigma$ pointwise such that the following diagram commutes:

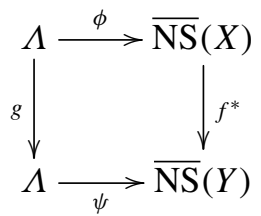


More generally, if $X$ is a K3 surface that is lattice-polarized by $(\Lambda, \Sigma)$, and if $S \subset \Sigma$ is a subset that spans the $\mathbb{Q}$-vector space $\Sigma \otimes \mathbb{Q}$, then we also say that $X$ is lattice-polarized by $(\Lambda, S)$. Similarly, we may speak of the moduli space $\mathcal{M}_{\Lambda, S}:=\mathcal{M}_{\Lambda, \Sigma}$.

REMARK 1.2. By convention, we assume that the lattice $\Lambda$ has a class with positive norm and has a unique embedding in the $K 3$ lattice $E_{8}^{2} \oplus U^{3}$ (up to equivalence) in order to define the above moduli space (see $[30,58]$ ); here $E_{8}$ denotes the unique 8-dimensional negative definite even unimodular lattice and $U$ the hyperbolic lattice with Gram matrix $\left(\begin{array}{ll}0 & 1 \\ 1 & 0\end{array}\right)$. In all of the cases we consider, the lattice $\Lambda$ will satisfy these properties. If $S$ equals $\Lambda$ (or contains a set of generators of $\Lambda$ ) in Definition 1.1, then we obtain the moduli space $\mathcal{M}_{\Lambda}$ of $\Lambda$ polarized $\mathrm{K} 3$ surfaces. Furthermore, if the $\mathbb{Z}$-span of $S$ contains a positive class, then the cover $\mathcal{M}_{\Lambda, S} \rightarrow \mathcal{M}_{\Lambda}$ is finite.

Then our main theorem is as follows.

THEOREM 1.3. Let $F$ be a field of characteristic 0 . For any line of Table 1, there exists an explicit finite subset $S$ of $\Lambda$ such that the $G(F)$-orbits of an open subset of $V(F)$ are in canonical bijection with the $F$-points of an open subvariety of the moduli space $\mathcal{M}_{\Lambda, S}$ of $K 3$ surfaces lattice-polarized by $(\Lambda, S)$.

In each section, we will specify the relevant subset $S$ of $\Lambda$.

COROLlary 1.4. The moduli space $\mathcal{M}_{\Lambda, S}$ of $(\Lambda, S)$-polarized $K 3$ surfaces is unirational. In particular, the moduli space $\mathcal{M}_{\Lambda}$ of $\Lambda$-polarized K3 surfaces is unirational.

A number of the spaces in Table 1 have been studied previously, often from the point of view of invariants of group actions and not necessarily with a specific connection to K3 surfaces, and usually over an algebraically closed field. Some of these results show that many of the moduli spaces in Table 1 are actually rational over $\bar{F}$. For instance, [47] proves the rationality of Nos. 2, 16, and 18; the rationality of No. 1 follows from [42]. The rationality of No. 17 follows from the classical computation of invariants of cubic surfaces, and that of No. 19 from the invariant theory of the binary quintic. It is an interesting problem to determine exactly which of the spaces in Table 1 are rational over $\bar{F}$ or over $F$.

We note that Table 1 is not intended to be a complete classification of all orbit spaces that are birational to moduli spaces related to lattice-polarized K3 surfaces. For instance, one could consider the space $3 \otimes 3 \otimes 4$, whose elements define an 
Table 2. Representations whose orbits parametrize polarized K3 surfaces. (The cases of degrees 10 to 18 are due to Mukai.)

\begin{tabular}{ccc}
\hline Group $G^{\text {ss }}$ & Representation $V$ & Degree \\
\hline $\mathrm{SL}_{3}$ & $\operatorname{Sym}^{6}(3)$ & 2 \\
$\mathrm{SL}_{4}$ & $\operatorname{Sym}^{4}(4)$ & 4 \\
$\mathrm{SL}_{5}$ & $\operatorname{Sym}^{2}(5) \oplus \operatorname{Sym}^{3}(5)$ & 6 \\
$\mathrm{SL}_{3} \times \mathrm{SL}_{6}$ & $3 \otimes \operatorname{Sym}^{2}(6)$ & 8 \\
$\mathrm{SL}_{2}$ & $\mathrm{Sym}^{8}(2) \oplus \mathrm{Sym}^{12}(2)$ & 10 \\
$\mathrm{SL}_{8} \times \mathrm{SO}_{10}$ & $8 \otimes \mathrm{S}^{+}(16)$ & 12 \\
$\mathrm{SL}_{6} \times \mathrm{SL}_{6}$ & $6 \otimes \wedge^{2}(6)$ & 14 \\
$\mathrm{SL}_{4} \times \mathrm{Sp}_{6}$ & $4 \otimes \wedge_{0}^{3}(6)$ & 16 \\
$\mathrm{SL}_{3} \times G_{2}$ & $3 \otimes(14)$ & 18 \\
\hline
\end{tabular}

unordered set of six points in the plane, or via duality, a set of six lines; the double cover of the plane branched along the six lines is a K3 surface of Picard number 16 , so over $\bar{F}$ (but not $F$ ), there is a correspondence between these orbits and such K3 surfaces. Such K3 surfaces have been extensively studied in the past; for example, see $[46,48,56]$.

As mentioned earlier, in the case where $\Lambda(=\Sigma)$ has rank 1, several cases have been studied previously, and we have recorded them in Table 2. The first four cases are classical and are easily adapted to give the correct parametrization over any field, while the last five are more recent and arise in the beautiful work of Mukai [53]. It is an interesting problem to work out the appropriate forms of Mukai's representations so that they also parametrize polarized K3 surfaces over a general field.

Figure 1 shows how many of the cases from both Tables 1 and 2 are related. In particular, each arrow from a representation $V$ of the group $G$ to a representation $V^{\prime}$ of $G^{\prime}$ indicates that there is a group homomorphism $\tau: G \rightarrow G^{\prime}$ and a map $V \rightarrow V^{\prime}$ that is $G$-equivariant with respect to $\tau$. For each arrow, there is a map between the associated moduli spaces of lattice-polarized K3 surfaces as well as a reverse inclusion of the corresponding polarization lattices $\Lambda$ and $\Sigma$. The ranks of the polarization lattices $\Lambda$ are indicated in the first column.

Such explicit descriptions of the moduli spaces of lattice-polarized K3 surfaces also have several other potential applications. For example, these moduli spaces are related to Noether-Lefschetz divisors, which are special cycles on moduli spaces of polarized K3 surfaces (see, for example, [45]). There has also been a great deal of recent activity surrounding the Noether-Lefschetz conjecture [6, 49], and it would be interesting to extend the work of Greer et al. [37] on the GIT stability of the Mukai models to these spaces of lattice-polarized K3s. 


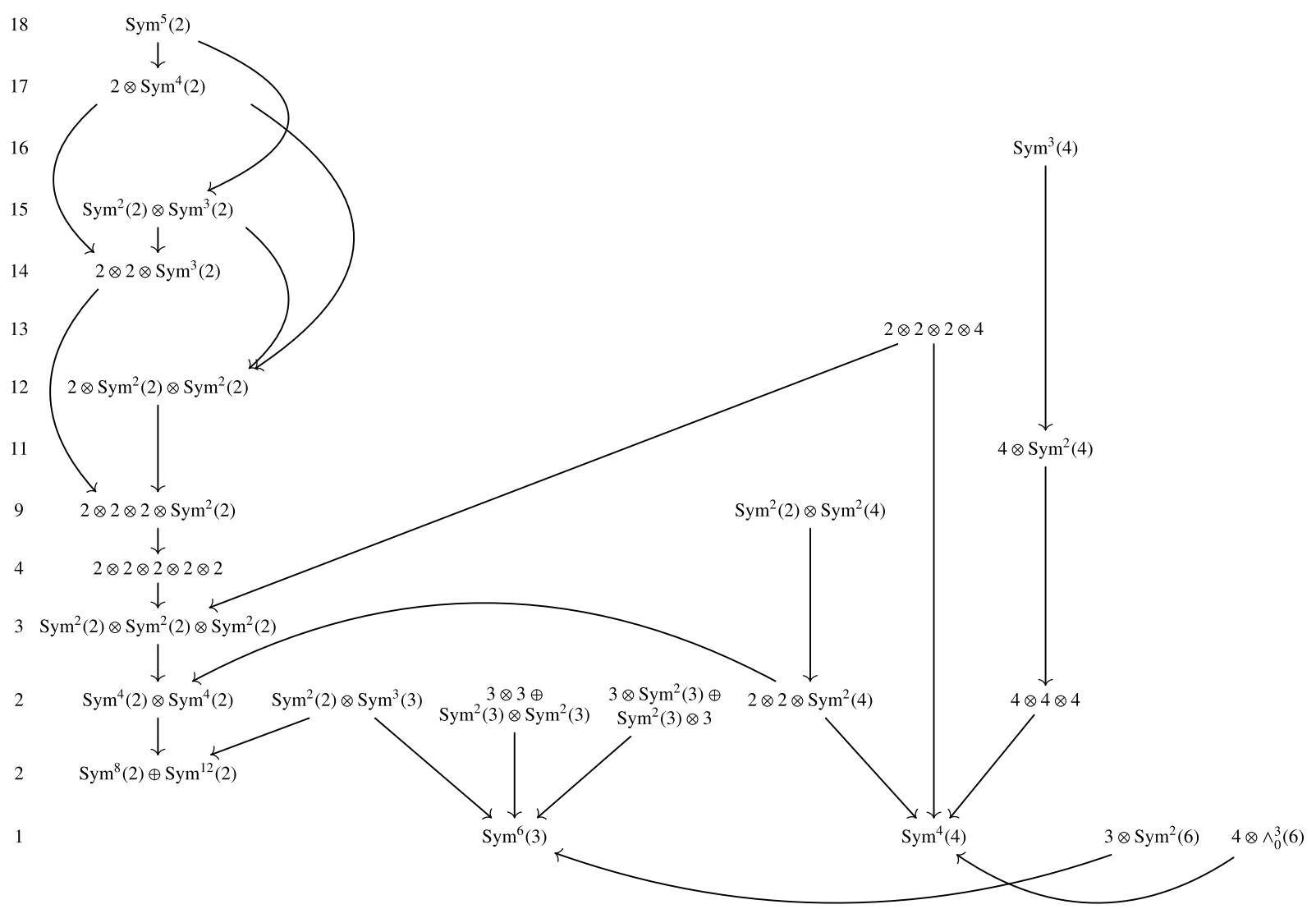

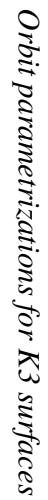

Figure 1. Covariance relations among orbit parametrizations of lattice-polarized K3 surfaces. 
In many of the new cases listed in Table 1, we also obtain natural automorphisms of the corresponding K3 surfaces. Due to their frequently extremely interesting and rich groups of automorphisms, K3 surfaces have provided a natural setting in recent years on which to study questions of dynamics (see, for example, $[20,51,60]$; for a nice survey, see [21]). In particular, there has been considerable interest in exhibiting positive entropy automorphisms of projective algebraic K3 surfaces. Recall that the entropy of an automorphism $\phi$ of a projective surface $X$ is defined to be $\log \lambda(\phi)$, where $\lambda(\phi)$ is the spectral radius of $\phi^{*}$ on $\overline{\mathrm{NS}}(X) \otimes \mathbb{R}$. When $X$ is defined over $\mathbb{C}$, this definition agrees with the topological entropy (see [21, Section 4.4.2]).

Recently, Oguiso [61] showed that any projective algebraic K3 with a fixedpoint-free automorphism of positive entropy must have Picard number at least 2. He also produced a family of examples with Picard number 2 by considering the Cayley K3 surfaces, that is, the K3 surfaces arising from Rubik's revenge (Line 6 of Table 1). More precisely, he proved that any K3 surface with NéronSeveri lattice exactly $\mathfrak{O}_{5}(2)$ has a fixed-point-free automorphism with entropy $\eta_{\mathrm{RR}}=6 \log ((1+\sqrt{5}) / 2)>0$. (See also [34].)

Our perspective on the Rubik's revenge case in Section 4 allows us to give a simpler proof of Oguiso's theorem, and in a stronger form, in Section 17. More precisely, we prove:

THEOREM 1.5. Let $X$ be a $K 3$ surface with line bundles $L_{1}$ and $L_{2}$ satisfying $L_{1}^{2}=L_{2}^{2}=4$ and $L_{1} \cdot L_{2}=6$. Assume that the projective embedding of $X$ corresponding to $L_{1}$ is smooth. Then $X$ has a fixed-point-free automorphism of entropy $\eta_{\mathrm{RR}}=6 \log ((1+\sqrt{5}) / 2) \approx 2.887>0$.

The key ingredient is the use of the hyperdeterminant. The hyperdeterminant is a generalization of the determinant for multidimensional matrices, which was introduced by Cayley and studied in depth by Gelfand et al. [35]. For most orbit parametrizations of rings and ideal classes by multidimensional matrices (for example, all those in $[7,8,75])$, the hyperdeterminant can be shown to equal the discriminant of the corresponding ring. For most orbit parametrizations of algebraic curves in terms of multidimensional matrices (for example, all those in $[4,14,16])$, the hyperdeterminant can be shown to equal the discriminant of the corresponding algebraic curve. Thus, for all these parametrizations of data associated to rings and curves by multidimensional matrices, the nonvanishing of the hyperdeterminant corresponds to the nondegeneracy of the associated ring and the nonsingularity of the associated curve, respectively.

The orbit parametrizations (of $\mathrm{K} 3$ surfaces by multidimensional matrices) considered in this paper yield a number of examples where the hyperdeterminant 
does not coincide with the discriminant. Indeed, in these cases, we show that the hyperdeterminant only divides the discriminant of the $\mathrm{K} 3$ surface, but is not equal to it. This raises the question as to the interpretation of the hyperdeterminant in these cases. We will prove that the nonvanishing of the hyperdeterminant corresponds precisely to an associated automorphism of the K3 surface being fixed-point-free. This interpretation is what consequently allows us to prove Theorem 1.5 for all nonsingular Cayley K3 surfaces.

An additional advantage of our method is that it also naturally extends to other cases. For example, we may use Line 9 of Table 1 together with our hyperdeterminant method to produce examples of K3 surfaces of rank 4 having many fixed-point-free automorphisms of positive entropy:

THEOREM 1.6. Let $X$ be a nonsingular K3 surface corresponding to a penteract. Then $X$ has a fixed-point-free automorphism with entropy $\log \left(\lambda_{\text {pent }}\right) \approx 2.717>$ 0 , where $\lambda_{\text {pent }}=4+\sqrt{13}+\sqrt{7+2 \sqrt{13}} \approx 15.145$ is the unique real root of $x^{4}-16 x^{3}+14 x^{2}-16 x+1$ greater than 1 .

In fact, we will show that these $\mathrm{K} 3$ surfaces coming from penteracts have infinitely many automorphisms of positive entropy. Recall that a Salem number is a real algebraic integer $\lambda>1$ whose conjugates other than $\lambda^{ \pm 1}$ lie on the unit circle; its irreducible minimal polynomial is then called a Salem polynomial (see [36] for a survey of problems involving Salem numbers). (We follow the convention of McMullen [51], where the set of Salem numbers includes quadratic integers with these properties. Another convention is to call such quadratic integers Pisot numbers.) The entropy of an automorphism of a projective K3 surface is either 0 or the logarithm of a Salem number [51, Section 3], and it is an interesting question as to which Salem polynomials arise from automorphisms of $\mathrm{K} 3$ surfaces (see, for example, [20, 51, 52, 63]).

We obtain a plethora of quadratic and quartic Salem polynomials from the automorphisms of the K3 surfaces arising from penteracts. In fact, we will demonstrate in Section 7.4 that, for a positive proportion of natural numbers $n$, both the polynomials $x^{2}-\left(4 n^{2} \pm 2\right) x+1$ and $x^{2}-\left(12 n^{2} \pm 2\right) x+1$ arise as Salem polynomials of automorphisms of the general K3 surface in the penteract family. As a consequence, it follows that all real quadratic fields occur as the splitting fields of Salem polynomials of automorphisms of general K3 surfaces in this family.

Other examples, similar to those mentioned in the preceding two theorems, will be constructed in Section 17; for example, we will construct fixed-point-free automorphisms on $\mathrm{K} 3$ surfaces in certain families having entropy equal to the logarithm of $3+2 \sqrt{2},(3+\sqrt{5}) / 2$, and $2+\sqrt{3}$, respectively. 
Outline. This paper is organized as follows. In Section 2, we give some background on lattices and $\mathrm{K} 3$ surfaces, as well as notation, that will be used throughout the paper. In Section 3, we then very briefly discuss some cases from Table 1 that are classical; for example, some of the moduli spaces where the general K3 has Picard rank 2. As seen in Figure 1, these classical examples often appear as covariants of other cases that we consider in this paper.

The bulk of this paper lies in Sections 4-16. In each of these sections, we prove Theorem 1.3 for the specified group $G$, representation $V$, lattice $\Lambda$, and subset $S$ of $\Lambda$ as listed in Table 1 . We begin each section with a construction of the $\mathrm{K} 3$ surfaces, and associated line bundles/divisors, obtained from a general $G(F)$ orbit of $V(F)$. In many of the sections, we also discuss various automorphisms of the relevant $\mathrm{K} 3$ surfaces arising from these constructions.

Finally, in Section 17, we consider some connections between these bijections and hyperdeterminants, as well as some applications to dynamics on K3 surfaces.

\section{Preliminaries}

2.1. Lattices. A lattice $\Lambda$ is a free abelian group of finite rank, equipped with a bilinear pairing $\langle\rangle:, \Lambda \times \Lambda \rightarrow \mathbb{Z}$. We will assume that the pairing is nondegenerate. A lattice is often described by the Gram matrix $G=\left(\left\langle v_{i}, v_{j}\right\rangle\right)_{i j}$ with respect to a basis $v_{1}, \ldots, v_{n}$ of $\Lambda$. The discriminant of the lattice is then $\operatorname{det}(G)$ and the discriminant group of $\Lambda$ is $A=\Lambda^{*} / \Lambda$, where $\Lambda^{*}$ is the dual lattice. It is equipped with a discriminant form $\phi: A \times A \rightarrow \mathbb{Q} / \mathbb{Z}$. The real signature of $\Lambda$ is the pair $\left(r_{+}, r_{-}\right)$consisting of the number of positive and negative eigenvalues of its Gram matrix $G$. We say that $\Lambda$ is positive definite (respectively, negative definite, or indefinite) if $r_{-}=0$ (respectively, $r_{+}=0$, or $\left.r_{+} r_{-} \neq 0\right)$. Similarly, one can define the integral $p$-adic signatures for every prime $p$, by considering $\Lambda_{p}=\Lambda \otimes \mathbb{Z}_{p}$ and its discriminant group $A_{\Lambda_{p}}$. These are invariants of the isomorphism class of the lattice.

We now record for use in this paper some useful theorems regarding embeddings and isomorphisms of lattices.

THEOREM 2.1. Let $\Lambda$ be an indefinite integral lattice of rank $n \geqslant 3$ and discriminant $d$. Assume that there is no odd prime $p$ such that $p^{n(n-1) / 2} \mid d$, and that $2^{n(n-3) / 2+\lfloor(n+1) / 2\rfloor} \nmid d$. Then the class number of the genus of $\Lambda$ is 1 . That is, $\Lambda$ is determined by the collection of its real and p-adic signatures.

This theorem is used in Table 1 to identify the various Néron-Severi lattices that arise in our orbit problems with direct sums of familiar lattices. For the lower rank examples, it is easy to give a direct identification; for higher rank, it suffices 
to compute the genus symbols of the Néron-Severi lattice (defined by a Gram matrix in the corresponding section), and match them with those of the lattices given in the table. For brevity, we omit these verifications in the text.

THEOREM 2.2 (Nikulin [59, Theorem 1.14.4]). Let $M$ be an even lattice with real signature $\left(t_{+}, t_{-}\right)$and discriminant form $\phi_{M}$, and let $\Lambda$ be an even unimodular lattice of signature $\left(s_{+}, s_{-}\right)$. Suppose that

(a) $t_{+}<s_{+}$;

(b) $t_{-}<s_{-}$;

(c) $\ell\left(A_{M_{p}}\right) \leqslant \operatorname{rank}(\Lambda)-\operatorname{rank}(M)-2$ for $p \neq 2$;

(d) One of the following condition holds at the prime 2.

(i) $\ell\left(A_{M_{2}}\right) \leqslant \operatorname{rank}(\Lambda)-\operatorname{rank}(M)-2$; or

(ii) $\ell\left(A_{M_{2}}\right)=\operatorname{rank}(\Lambda)-\operatorname{rank}(M)$ and $\phi_{M} \cong u_{2}^{+}(2) \oplus q^{\prime}$ or $\phi_{M} \cong v_{2}^{+}(2) \oplus$ $q^{\prime}$ for some $q^{\prime}$.

Then there exists a unique primitive embedding of $M$ into $\Lambda$.

Here, $\ell(A)$ denotes the smallest number of generators of the group $A$, and $u_{2}^{+}(2)$ and $v_{2}^{+}(2)$ are specific discriminant forms on certain finite 2-groups (see [59]). This theorem is the key ingredient in checking that our lattices have unique primitive embeddings in the $\mathrm{K} 3$ lattice. We leave the routine verification to the interested reader.

2.2. K3 surfaces. In this subsection, we first recall some basics of $K 3$ surfaces and explain the existence of a coarse moduli space $\mathcal{M}_{\Lambda, S}$ for a lattice $\Lambda$ as in Remark 1.2 and a subset $S$ of $\Lambda$. For simplicity, we work over the complex numbers, though it is possible also to give an algebraic description of latticepolarized K3 surfaces [5].

A K 3 surface $X$ over $F$ is a projective algebraic nonsingular surface with trivial canonical bundle and $\mathrm{h}^{1}\left(X, \mathcal{O}_{X}\right)=0$. The cohomology group $\mathrm{H}^{2}(X, \mathbb{Z})$, equipped with the cup product form, is a 22-dimensional lattice that is abstractly isomorphic to the $K 3$ lattice $\Lambda_{K 3}:=E_{8}^{2} \oplus U^{3}$, where $E_{8}$ is the 8-dimensional negative definite even unimodular lattice and $U$ is the hyperbolic lattice with Gram matrix $\left(\begin{array}{ll}0 & 1 \\ 1 & 0\end{array}\right)$. The Néron-Severi group $\overline{\mathrm{NS}}(X)$ as defined in the introduction is a primitive sublattice of the K3 lattice, with signature $(1, \rho-1)$.

We start with the notion of a marked $\Lambda$-polarized $\mathrm{K} 3$ surface. Pick an embedding $\Lambda \hookrightarrow \Lambda_{K 3}$ (it does not matter which, since all embeddings are 
equivalent by assumption). A marking is an isomorphism $\phi: \mathrm{H}^{2}(X, \mathbb{Z}) \rightarrow \Lambda_{K 3}$ such that $\phi^{-1}(\Lambda) \subset \mathrm{NS}(X)$. For such a marked polarized K3 surface, the class of a regular 2-form $\omega$ on $X$ maps under $\phi$ to an element $z \in \Lambda^{\perp} \otimes \mathbb{C}$, since $z$ pairs to zero with the algebraic classes. Furthermore, it is easy to see from Hodge theory that $\langle z, z\rangle>0$ and $\langle z, \bar{z}\rangle=0$. Therefore, we have $z \in \Omega$ where $\Omega$ is an open subset of the quadric cone in $\Lambda^{\perp} \otimes \mathbb{C}$ defined by these two conditions. Since the form is unique up to scaling, we obtain a well-defined element of $\mathbb{P}(\Omega)$. The map taking $(X, \phi)$ to $z$ is called the period mapping. It can be shown that it yields an isomorphism between the moduli space of marked ample $\Lambda$-polarized K3 surfaces and the complement $\Omega^{0}$ of a union of hyperplanes in $\Omega$.

To remove the marking, let $\Gamma(\Lambda)$ be the group

$$
\Gamma(\Lambda)=\left\{\sigma \in O\left(\Lambda_{K 3}\right): \sigma(v)=v \text { for all } v \in \Lambda\right\} .
$$

Then an element $\sigma \in \Gamma(\Lambda)$ acts on the moduli space by sending $(X, \phi)$ to $(X$, $\phi \circ \sigma)$, which gives an isomorphism of the polarized K3 surfaces. Let $\Gamma_{\Lambda}$ be the image of $\Gamma(\Lambda)$ in $O\left(\Lambda^{\perp}\right)$. Then the moduli space of $\Lambda$-polarized K3 surfaces is obtained by taking the quotient by the group action of $\Gamma(\Lambda)$. It establishes an isomorphism with the period domain, obtained by taking the quotient $\Omega^{0} / \Gamma_{\Lambda}$. For more details, we refer the reader to [30].

In this paper, we will require a minor modification of this construction. Namely, we do not quotient by the pointwise stabilizer of $\Lambda$, but only by the pointwise stabilizer of $S$. Let

$$
\Gamma(\Lambda, S)=\left\{\sigma \in O\left(\Lambda_{K 3}\right): \sigma(\Lambda)=\Lambda \text { and } \sigma(s)=s \text { for any } s \in S\right\} .
$$

Then $\Gamma(\Lambda, S)$ contains $\Gamma(\Lambda)$ as a subgroup, and is generally strictly larger. (However, $\Gamma(\Lambda)$ is a finite-index subgroup of $\Gamma(\Lambda, S)$ if $S$ (or its span) contains a positive/ample class.) Let $\Gamma_{\Lambda, S}$ be its image in $O\left(\Lambda^{\perp}\right)$. The moduli space of $(\Lambda, S)$-polarized $\mathrm{K} 3$ surfaces is obtained by taking the quotient of the fine moduli space of ample marked $\Lambda$-polarized $\mathrm{K} 3$ surfaces by $\Gamma(\Lambda, S)$. From the period mapping, it follows that the dimension of the space $\mathcal{M}_{\Lambda, S}$ (when $S$ contains a positive divisor) is $20-\operatorname{rank} \Lambda$.

We note here a lemma of Nikulin [57, Lemma 3], which will be very useful in the determination of Néron-Severi groups of the K3 surfaces studied in this paper.

LEMMA 2.3 (Nikulin). Let $X$ be a K3 surface, and $E_{1}, \ldots, E_{n}$ disjoint smooth rational curves on $X$ such that $\frac{1}{2}\left(E_{1}+\cdots+E_{n}\right) \in \overline{\mathrm{NS}}(X)$. Then $n \in\{0,8,16\}$.

The proof is a simple calculation of the Euler characteristic of the double cover of $X$ branched along the divisor $\sum E_{i}$. 
2.3. Notation and conventions. We make some brief remarks on the notation and conventions used in this paper.

- Unless otherwise stated, we will work over a field $F$ of characteristic 0 . We expect that most of the results also hold for nonsupersingular K3 surfaces in positive characteristic larger than 3 .

- For a vector space $V$, the notation $\operatorname{Sym}^{n}(V)$ denotes the $n$th symmetric power of $V$ as a quotient of $V^{\otimes n}$. However, since we will be working over a field of characteristic 0 , this space is canonically isomorphic to the subspace $\operatorname{Sym}_{n}(V)$ of $V^{\otimes n}$ and, in fact, it will usually be more natural for us to view it as the subspace.

- If $V$ is a representation of a group $G$, we will sometimes consider an action of $\mathbb{G}_{m} \times G$ on $V$, where $\mathbb{G}_{m}$ acts by scaling.

- We will be denoting various K3 surfaces using indices; in each such case, any permutation of the subscripts will denote the same surface; for example, $X_{123}$ and $X_{132}$ will refer to the same surface.

- We pass between line bundles and divisors on our K3 surfaces freely, and we will often use additive notation to denote the tensor product of line bundles. When working with relations among line bundles, we also use $=$ to denote an isomorphism (or equivalence among divisors).

- Multilinear forms play a large role in many of our constructions. For example, for vector spaces $V_{1}, V_{2}$, and $V_{3}$, we sometimes denote an element $A$ of $V_{1} \otimes$ $V_{2} \otimes V_{3}$ as the trilinear form $A(\cdot, \cdot, \cdot)$, where each $\cdot$ may also be replaced by an element of the appropriate dual vector space $V_{i}^{\vee}$. By abuse of notation, we may also allow points of the projective space $\mathbb{P}\left(V_{i}^{\vee}\right)$ as entries in the multilinear form $A$ when we are only asking about the vanishing or nonvanishing of $A$. Finally, the notation $A\lrcorner x$ for an element $x \in V_{2}^{\vee}$ is just $A(\cdot, x, \cdot)$, for example.

- When discussing the induced action of an automorphism of a K3 surface on the Néron-Severi group, the matrices will act on row vectors. In particular, if $Q$ is the matrix of the quadratic form representing the Néron-Severi lattice, then we have $M Q M^{t}=Q$ for the matrix $M$ of any automorphism.

\section{Some classical moduli spaces for K3 surfaces with low Picard number}

We first recall some of the classical cases listed in the first few entries of Table 1. All but the last of them have Picard number 2, leading to a moduli space of dimension $20-2=18$. In each case below, we see directly that the moduli space 
is unirational and the points in an open subset correspond bijectively to orbits of a suitable representation of a reductive group. The excluded locus in each case is a union of Noether-Lefschetz divisors (in the sense of [49]) on the corresponding moduli space of lattice-polarized K3 surfaces.

For the cases with Picard number 2, the generic Néron-Severi lattice is even, of signature $(1,1)$ and of (absolute) discriminant $D$. This lattice coincides with the lattice underlying the quadratic ring $\mathfrak{O}_{D}$ of discriminant $D$ equipped with twice the norm bilinear form, that is, the form $\langle u, v\rangle=N(u+v)-N(u)-N(v)$.

3.1. Elliptic surfaces with section. The simplest indefinite even lattice is the hyperbolic plane $U$ of discriminant 1 . The moduli space of K3 surfaces latticepolarized by $U$ is the same as that of elliptic surfaces with section. Over a field of characteristic not 2 or 3, we may write the Weierstrass equation of such a surface as

$$
y^{2}=x^{3}+a_{4}(t) x+a_{6}(t),
$$

with $a_{4}(t)$ and $a_{6}(t)$ polynomials of degree at most 8 and 12, respectively. (For such a Weierstrass equation to describe a K3 rather a rational surface, we also need $\operatorname{deg}\left(a_{4}\right)>4$ or $\operatorname{deg}\left(a_{6}\right)>6$.) Once we quotient by Weierstrass scaling ( $x$, $y) \rightarrow\left(\lambda^{4} x, \lambda^{6} y\right)$ and the $\mathrm{PGL}_{2}$ action on the base $\mathbb{P}_{t}^{1}$, we obtain a moduli space of dimension $9+13-1-3=18$, as expected. This moduli space $\mathcal{M}_{U}$ is clearly unirational, and corresponds to the representation $\operatorname{Sym}^{8}(2) \oplus \operatorname{Sym}^{12}(2)$.

3.2. Double covers of $\mathbb{P}^{\mathbf{1}} \times \mathbb{P}^{\mathbf{1}}$. The second discriminant we need to consider is 4 , corresponding to the lattice $U(2)$. The corresponding $\mathrm{K} 3$ surfaces are double covers of $\mathbb{P}^{1} \times \mathbb{P}^{1}$, branched along a bidegree $(4,4)$ curve. The pullbacks of the two hyperplane classes give us line bundles $L_{1}$ and $L_{2}$ with $L_{1}^{2}=L_{2}^{2}=0$ and $L_{1} \cdot L_{2}=2$. Either of the projections to $\mathbb{P}^{1}$ is a genus one fibration, and exhibits the surface as an elliptic surface with a 2-section. The moduli space is birational to the space of orbits of $\mathbb{G}_{m} \times \mathrm{GL}_{2}^{2}$ on $\mathrm{Sym}^{4}(2) \otimes \operatorname{Sym}^{4}(2)$.

3.3. Hypersurfaces of bidegree $(2,3)$ in $\mathbb{P}^{1} \times \mathbb{P}^{2}$. A smooth hypersurface of bidegree $(2,3)$ in $\mathbb{P}^{1} \times \mathbb{P}^{2}$ is a $\mathrm{K} 3$ surface. It has two line bundles $L_{1}$ and $L_{2}$ which are pullbacks of the hyperplane classes, and satisfy $L_{1}^{2}=0, L_{2}^{2}=2$, and $L_{1} \cdot L_{2}=3$. The generic Néron-Severi lattice of this family

$$
\left(\begin{array}{ll}
0 & 3 \\
3 & 2
\end{array}\right)
$$

has discriminant 9. The moduli space is birational to the quotient of $\operatorname{Sym}^{2}(2) \otimes$ $\operatorname{Sym}^{3}(3)$ by $\mathbb{G}_{m} \times \mathrm{GL}_{2} \times \mathrm{GL}_{3}$. 


\subsection{Complete intersection of bidegree $(1,1)$ and $(2,2)$ hypersurfaces} in $\mathbb{P}^{2} \times \mathbb{P}^{2}$. Next, we consider K3 surfaces given as the smooth complete intersection of bidegree $(1,1)$ and $(2,2)$ forms in $\mathbb{P}^{2} \times \mathbb{P}^{2}$. This time, the pullbacks $L_{1}$ and $L_{2}$ of the two line bundles satisfy $L_{i}^{2}=2$ (since the intersection of two lines on one of the $\mathbb{P}^{2}$ 's specifies a point, whence $L_{i}^{2}$ is obtained by computing the intersection number of a line and a conic). Similarly, we check that $L_{1} \cdot L_{2}=4$, from the intersection number of bidegree $(1,1)$ and $(2,2)$ curves on $\mathbb{P}^{1} \times \mathbb{P}^{1}$. Therefore, the generic Néron-Severi lattice has Gram matrix

$$
\left(\begin{array}{ll}
2 & 4 \\
4 & 2
\end{array}\right)
$$

with discriminant 12 . The moduli space is birational to the quotient of $3 \otimes 3 \oplus$ $\operatorname{Sym}^{2}(3) \otimes \operatorname{Sym}^{2}(3)$ by $\mathbb{G}_{m} \times\left(\mathbb{G}_{a}^{9} \rtimes \mathrm{GL}_{3}^{2}\right)$, where $\mathbb{G}_{a}^{9}$ acts by adding to the $(2,2)$ form the product of the given bidegree $(1,1)$ form with another bidegree $(1,1)$ form.

\subsection{Complete intersection of bidegree $(1,2)$ and $(2,1)$ hypersurfaces in} $\mathbb{P}^{2} \times \mathbb{P}^{2}$. Finally, consider K3 surfaces given as the smooth complete intersection of bidegree $(1,2)$ and $(2,1)$ forms in $\mathbb{P}^{2} \times \mathbb{P}^{2}$. As in the case of discriminant 12 above, we obtain the generic Néron-Severi lattice

$$
\left(\begin{array}{ll}
2 & 5 \\
5 & 2
\end{array}\right)
$$

of discriminant 21 . The moduli space is birational to the quotient of $3 \otimes \operatorname{Sym}^{2}(3) \oplus$ $\operatorname{Sym}^{2}(3) \otimes 3$ by $\mathrm{GL}_{3}^{2}$.

3.6. Hypersurfaces of tridegree $(2,2,2)$ in $\mathbb{P}^{\mathbf{1}} \times \mathbb{P}^{\mathbf{1}} \times \mathbb{P}^{\mathbf{1}}$. Finally, we consider $\mathrm{K} 3$ surfaces defined by the vanishing of a tridegree $(2,2,2)$ form on $\mathbb{P}^{1} \times \mathbb{P}^{1} \times \mathbb{P}^{1}$. The three line bundles obtained from pulling back $\mathcal{O}_{\mathbb{P}^{1}}(1)$ have intersection matrix

$$
\left(\begin{array}{lll}
0 & 2 & 2 \\
2 & 0 & 2 \\
2 & 2 & 0
\end{array}\right) \text {. }
$$

The moduli space is birational to the quotient of $\operatorname{Sym}^{2}(2) \otimes \operatorname{Sym}^{2}(2) \otimes \operatorname{Sym}^{2}(2)$ by $\mathbb{G}_{m} \times \mathrm{GL}_{2}^{3}$.

\section{Rubik's revenge: $4 \otimes 4 \otimes 4$}

We begin with a space of K3 surfaces that has been well studied in the classical literature in algebraic geometry $[23,41,65,68]$, as well as more recently $[4,34$, 
61]: that of determinantal quartics. However, our perspective is slightly different, allowing us to unify several existing results in the literature; in particular, we classify orbits on the space of $4 \times 4 \times 4$ cubical matrices over a general field $F$ in terms of moduli spaces of certain lattice-polarized K3 surfaces of Picard rank 2 over $F$, allowing general ADE singularities. The constructions we use here will also help prepare us for the larger rank cases to follow in later sections.

THEOREM 4.1. Let $V_{1}, V_{2}$ and $V_{3}$ be 4-dimensional vector spaces over $F$. Let $G^{\prime}=\mathrm{GL}\left(V_{1}\right) \times \mathrm{GL}\left(V_{2}\right) \times \mathrm{GL}\left(V_{3}\right)$, and let $V$ be the representation $V_{1} \otimes V_{2} \otimes V_{3}$ of $G^{\prime}$. Let $G$ be the quotient of $G^{\prime}$ by the kernel of the multiplication map on scalars, that is, $\mathbb{G}_{m} \times \mathbb{G}_{m} \times \mathbb{G}_{m} \rightarrow \mathbb{G}_{m}$. Let $\Lambda$ be the lattice whose Gram matrix is

$$
\left(\begin{array}{ll}
4 & 6 \\
6 & 4
\end{array}\right)
$$

and let $S=\left\{e_{1}, e_{2}\right\}$. Then the $G(F)$-orbits of an open subset of $V(F)$ are in bijection with the $F$-points of an open subvariety of the moduli space $\mathcal{M}_{\Lambda, S}$ of nonsingular $K 3$ surfaces lattice-polarized by $(\Lambda, S)$.

4.1. Construction of $\mathbf{K} 3$ surfaces. We first describe the construction of a K3 surface from an element $A \in V=V_{1} \otimes V_{2} \otimes V_{3}$, where $V_{1}, V_{2}$, and $V_{3}$ are 4dimensional $F$-vector spaces. With bases for $V_{1}, V_{2}$, and $V_{3}$, we may view $A$ as a $4 \times 4 \times 4$ cubical matrix $\left(a_{i j k}\right)_{1 \leqslant i, j, k \leqslant 4}$ with entries in $F$. For any $x \in V_{1}^{\vee}$, we may obtain a $4 \times 4$ matrix $A\lrcorner x$ of linear forms in $x$. The determinant of this matrix is a form $f$ of degree 4 in four variables, and its vanishing locus is a quartic surface $X_{1}$ in $\mathbb{P}\left(V_{1}^{\vee}\right) \cong \mathbb{P}^{3}$. We restrict our attention to the general case where $X_{1}$ has at most simple isolated singularities, which are thus $\mathrm{K} 3$ surfaces; in this case, we say that $A$ is nondegenerate.

Similarly, we may repeat this construction in the other two directions (replacing $V_{1}$ with $V_{2}$ or $\left.V_{3}\right)$ to obtain two more K3 surfaces $X_{2} \subset \mathbb{P}\left(V_{2}^{\vee}\right)$ and $X_{3} \subset \mathbb{P}\left(V_{3}^{\vee}\right)$. We claim that these three $\mathrm{K} 3$ surfaces are birational to each other. For example, to exhibit the map $X_{1} \rightarrow X_{2}$, we view $A$ as a trilinear form on $V_{1}^{\vee} \times V_{2}^{\vee} \times V_{3}^{\vee}$. Then let

$$
X_{12}:=\left\{(x, y) \in \mathbb{P}\left(V_{1}^{\vee}\right) \times \mathbb{P}\left(V_{2}^{\vee}\right): A(x, y, \cdot)=0\right\} .
$$

Then we observe that the projections of $X_{12}$ to $\mathbb{P}\left(V_{1}^{\vee}\right)$ and $\mathbb{P}\left(V_{2}^{\vee}\right)$ are $X_{1}$ and $X_{2}$, respectively, thereby giving a correspondence between $X_{1}$ and $X_{2}$. In particular, given a point $x \in X_{1}$, the determinant of $\left.A\right\lrcorner x$ vanishes, and the $y \in X_{2}$ such that $(x, y) \in X_{12}$ are exactly those $y$ (up to scaling) in the kernel of $\left.A\right\lrcorner x$ in $V_{2}^{\vee}$. We claim that if the kernel is at least 2-dimensional, then the point $x \in X_{1}$ is a singular point. Indeed, if all of the $3 \times 3$ minors $A_{s t}^{*}(x)$ of $\left.A\right\lrcorner x$ vanish, for $1 \leqslant s, t \leqslant 4$, 
then so do the partial derivatives

$$
\frac{\partial f}{\partial x_{i}}(x)=\sum_{s, t} c_{i s t} A_{s t}^{*}(x),
$$

where $c_{i s t}=(-1)^{s+t} a_{i s t}$. Hence if $x \in X_{1}$ is nonsingular, then the kernel of $\left.A\right\lrcorner x$ is exactly 1-dimensional. Generically, if the kernel of $A\lrcorner x$ is 2-dimensional, then $x$ gives an isolated singularity of $X_{1}$, which we call a rank singularity of $X_{1}$. (Furthermore, if the kernel is 3-dimensional, then the surface $X_{1}$ is a rational surface.) (It is possible for $X_{1}$ to have isolated singularities that are not rank singularities; see Section 17.1 for a further discussion of singularities on these surfaces.)

This describes a map $\psi_{12}: X_{1} \rightarrow X_{2}$, and it is easy to see that it is generically an isomorphism, as we may construct the inverse map $\psi_{12}^{-1}=\psi_{21}: X_{2}-\rightarrow X_{1}$ in the analogous manner. Similarly, we have maps $\psi_{i j}=\psi_{j i}^{-1}$ for all $1 \leqslant i \neq j \leqslant 3$. However, we note that the composition $\Phi:=\psi_{31} \circ \psi_{23} \circ \psi_{12}$ is not the identity! The resulting automorphism will be discussed further in Section 4.4.

The isomorphism classes of the $\mathrm{K} 3$ surfaces $X_{i}$ and maps $\psi_{i j}$ are invariant under the action of the group $G$. As there is a finite stabilizer group for a generic point in $V$ (in fact, the stabilizer is trivial; see Lemma 4.3 below), the dimension of the moduli space of K3 surfaces obtained in this way is $64-46=18$.

4.2. Néron-Severi lattice. We will see below that the Néron-Severi lattices of these K3 surfaces all contain a particular 2-dimensional lattice with Gram matrix

$$
\left(\begin{array}{ll}
4 & 6 \\
6 & 4
\end{array}\right)
$$

The space of K3 surfaces with this lattice polarization has dimension $20-2=18$. Therefore, we see that the Néron-Severi lattice of a generic K3 surface in this family will be this 2-dimensional lattice above.

To understand the Néron-Severi group of a K3 surface in our family, say $X_{1}=$ $X_{1}(A)$ for a particular choice of $A$ and bases for the vector spaces, we proceed as follows. Let $W$ be the vanishing locus in $X_{1}$ of the top left $3 \times 3$ minor of $A(x, \cdot, \cdot)$; note that $W$ contains, in particular, all the isolated rank singularities of $X_{1}$. The maps $\psi_{12}$ and $\psi_{13}$ can be expressed by the minors of the last row and column, respectively, of $A(x, \cdot, \cdot)$ (with the appropriate signs). Note that each of these two sets of minors contains the top $3 \times 3$ minor of $A(x, \cdot, \cdot)$. Hence we see that $W$ contains a divisor equivalent to $C=\psi_{12}^{*}\left(L_{2}\right)$, where $L_{2}$ is the hyperplane class of $X_{2} \subset \mathbb{P}\left(V_{2}^{\vee}\right)$, and similarly $W$ contains a divisor equivalent to $D=\psi_{13}^{*}\left(L_{3}\right)$. By direct calculation, we observe that the scheme $W$ is reducible, 
and generically decomposes into two components, which must therefore be $C$ and $D$. In the Néron-Severi lattice, we therefore have

$$
3 H=W=C+D+\sum_{i} E_{i},
$$

where $H=L_{1}$ is the hyperplane class of $X_{1}$ and the $E_{i}$ are the exceptional divisors over the isolated rank singularities. In the generic case, there are no exceptional divisors $E_{i}$.

We now compute the intersection numbers involving $H$ and $C$, assuming there are no exceptional divisors. We have $H^{2}=4$, and $C^{2}=\left\langle\psi_{12}^{*}\left(L_{2}\right), \psi_{12}^{*}\left(L_{2}\right)\right\rangle=$ $L_{2}^{2}=4$ and similarly $D^{2}=4$. So we obtain $36=(3 H)^{2}=C^{2}+D^{2}+2 C \cdot D=$ $4+2 C \cdot D$, giving $C \cdot D=14$. Therefore, $C \cdot 3 H=C \cdot(C+D)=4+14=18$, leading to $C \cdot H=6$. The divisors $H$ and $C$ thus have the intersection matrix (1).

Proposition 4.2. The Picard group of the K3 surface $X_{1}$ corresponding to a very general point (in the moduli space of Rubik's revenge cubes) is generated by the classes of $C$ and $H$.

Proof. The discriminant of the lattice generated by $C$ and $H$ is $20=2^{2} \cdot 5$, so it is enough to check that it is 2 -saturated. Since $C / 2$ and $H / 2$ have self-intersection 1 , which is odd, neither of these classes are in NS $(X)$. Similarly, $(C+H) / 2$ has self-intersection 5. Therefore, $\mathrm{NS}(X)=\mathbb{Z} C+\mathbb{Z} H$.

LEMMA 4.3. A quartic surface $X=X_{1}$ associated to a very general point in the moduli space of $(\Lambda, S)$-polarized $K 3$ surfaces has no linear automorphisms (that is, induced from $\mathrm{PGL}_{4}$ ) other than the identity.

Proof. From [58], we have the following description of the automorphism group. Let $O^{+}(\mathrm{NS}(X))$ be the set of isometries of the Néron-Severi lattice which preserve the Kähler cone and $O_{\omega}(\mathrm{T}(X))$ be the set of isometries of the transcendental lattice which preserve the period $\omega$ of the K3 surface, up to \pm 1 . Then

$$
\operatorname{Aut}(X) \cong\left\{(g, h) \in O^{+}(\mathrm{NS}(X)) \times O_{\omega}(\mathrm{T}(X)): \bar{g}=\bar{h}\right\},
$$

where ${ }^{-}$refers to the natural morphisms from the orthogonal groups of the lattices $\mathrm{NS}(X)$ or $\mathrm{T}(X)$ to their discriminant groups, which are isomorphic.

For a general element of the moduli space, the only Hodge isometries of the transcendental lattice are $h= \pm \mathrm{Id}$. Suppose $g$ preserves the class of $H$ and $g(C)=m C+n H$ for some $m, n \in \mathbb{Z}$. Since $g(H) \cdot g(C)=H \cdot C=6$, we obtain $6 m+4 n=6$. Similarly, $g(C) \cdot g(C)=C \cdot C=4$ gives $4 m^{2}+4 n^{2}+12 m n=4$. Combining these, we get $(m, n)=(1,0)$ or $(-1,3)$. In the first case, we have 
$g=\mathrm{Id}$ and by the condition on the discriminant group (which is not 2-torsion), we see that $h=\mathrm{Id}$ is forced, leading to the identity automorphism of $X$. In the second case, we see that $C$ and $D$ are switched under $g$; however, since $g$ does not act by \pm 1 on the discriminant group, which is generated by $(H+C) / 10$ and $H / 2$, it does not give an automorphism of $X$.

COROLlARY 4.4. The stabilizer of the action of $G$ on $V$ is generically trivial.

Proof. If $g=\left(g_{1}, g_{2}, g_{3}\right)$ stabilizes $v \in V$, then $g_{i}$ gives a linear automorphism of $X_{i}$ for each $i$. Therefore, generically $g=1$.

4.3. Moduli problem. This subsection contains the proof of Theorem 4.1. We have already given a construction from an element of $V_{1} \otimes V_{2} \otimes V_{3}$ to a $(\Lambda, S)$ polarized K3 surface. The bulk of the proof is to show the reverse construction. We start with a well-known lemma; a simple proof may be found in [50] with more details in [66]. We include this proof below, since it is a useful template for the proofs of this section.

LEMMA 4.5. Let $(X, L)$ be a generic point in the moduli space $\mathcal{M}_{4}$ of $K 3$ surfaces equipped with a line bundle $L$ with $L^{2}=4$. Then the linear system $|L|$ embeds $X$ as a quartic surface in $\mathbb{P}^{3}$.

Proof. By Riemann-Roch, $\mathrm{h}^{0}(L)+\mathrm{h}^{2}(L) \geqslant 4$, so $L$ or $-L$ is effective. We may assume the former without loss of generality. For a generic point in the moduli space, the linear system $|L|$ contains an irreducible curve $C$. By Bertini's theorem, we may even assume $C$ is smooth. It is not difficult to show that $\mathrm{h}^{1}(L)=0$, so $\mathrm{h}^{0}(L)=4$. Therefore, the associated morphism $\phi_{L}$ maps $X$ to $\mathbb{P}^{3}$. Either (i) $\operatorname{deg}(\phi)=1$ and the image is a quartic surface in $\mathbb{P}^{3}$, or (ii) $\operatorname{deg}(\phi)=2$ and the image is a quadric surface, and the curve $C$ is a double cover of a plane conic branched at 8 points, and therefore a hyperelliptic curve of genus 3 . The second case does not occur generically (see, for instance, the argument in [40, Remark 2.3.8 and Example 2.3.9] or [1, Exp. VI]), and leads to an increase in the Picard number.

REMARK 4.6. The locus of K3 surfaces for which $|L|$ does not contain an irreducible curve (alternatively, has a base locus, necessarily a smooth rational curve) is a Noether-Lefschetz divisor. In this unigonal case, the complete linear system $|L|$ describes $X$ as an elliptic surface over a twisted cubic in $\mathbb{P}^{3}$. The hyperelliptic or digonal case (ii) in the proof above also corresponds to a NoetherLefschetz divisor. 
Most of the proof of Theorem 4.1 will be established in the following result, which we state separately, since it will also be useful in subsequent sections.

THEOREM 4.7. Let $X$ be a K3 surface equipped with two line bundles $L_{1}, L_{2}$ such that $L_{1}^{2}=L_{2}^{2}=4$ and $L_{1} \cdot L_{2}=6$. Assume in addition that $L_{1}$ and $L_{2}$ correspond to effective divisors $C_{1}$ and $C_{2}$ on $X$ that induce maps to $\mathbb{P}^{3}$ whose images are normal quartic surfaces. Then $X$ arises from $a 4 \times 4 \times 4$ matrix via the construction of Section 4.1.

Proof. We consider $X$ as a quartic surface in $\mathbb{P}^{3}$, embedded through the linear system $\left|L_{1}\right|$. Then $L_{2}$ corresponds to a nonhyperelliptic curve $C$ on $X$ of genus 3 . Equivalently, $C$ is projectively normal. It is well known that the sheaf $\mathcal{O}_{X}(C)$ is arithmetically Cohen-Macaulay (see [4] and for more general hypotheses, [31, Ch. 4]). Therefore, there is an exact sequence

$$
0 \rightarrow \mathcal{O}_{\mathbb{P}^{3}}(-1)^{4} \rightarrow \mathcal{O}_{\mathbb{P}^{3}}^{4} \rightarrow j_{*} L_{2} \rightarrow 0,
$$

where $j: X \rightarrow \mathbb{P}^{3}$ is the embedding as a quartic surface. Taking the long exact sequence, and using $h^{0}\left(\mathbb{P}^{3}, \mathcal{O}_{\mathbb{P}^{3}}(-1)\right)=h^{1}\left(\mathbb{P}^{3}, \mathcal{O}_{\mathbb{P}^{3}}(-1)\right)=0$, we have an identification of $\mathrm{h}^{0}\left(\mathbb{P}^{3}, \mathcal{O}_{\mathbb{P}^{3}}^{4}\right)$ with $\mathrm{h}^{0}\left(X, L_{2}\right)$.

Next, tensoring with the exact $L_{1}$ and taking cohomology, we obtain

$$
0 \rightarrow \mathrm{H}^{0}\left(\mathcal{O}_{\mathbb{P}^{3}}\right)^{4} \rightarrow \mathrm{H}^{0}\left(\mathcal{O}_{\mathbb{P}^{3}}(1)\right)^{4} \rightarrow \mathrm{H}^{0}\left(j_{*} L_{2} \otimes L_{1}\right) \rightarrow \mathrm{H}^{1}\left(\mathcal{O}_{\mathbb{P}^{3}}\right)^{4}=0 .
$$

Thus we obtain a surjective map

$$
\mu: \mathrm{H}^{0}\left(X, L_{1}\right) \otimes \mathrm{H}^{0}\left(X, L_{2}\right) \rightarrow \mathrm{H}^{0}\left(X, L_{1} \otimes L_{2}\right) .
$$

Since each $\mathrm{H}^{0}\left(X, L_{i}\right)$ is 4-dimensional, the map has a 4-dimensional kernel. Thus, we obtain a $4 \times 4 \times 4$ matrix, giving rise to a determinantal representation of $X$.

Proof of Theorem 4.1. Given a $4 \times 4 \times 4$ tensor, we have already seen how to produce a K3 surface $X$ with two line bundles $L_{1}$ and $L_{2}$ with the required pairing matrix.

Conversely, given a K3 surface $X$ with line bundles $L_{1}$ and $L_{2}$, Riemann-Roch shows that either $L_{1}$ or its inverse is effective, and similarly for $L_{2}$. Normalizing so that $L_{1}$ and $L_{2}$ are effective, we see that generically (in the moduli space of lattice-polarized $\mathrm{K} 3$ surfaces) each gives a quartic embedding to $\mathbb{P}^{3}$. Therefore, we may use the result of Theorem 4.7 to produce a $4 \times 4 \times 4$ tensor.

It remains to show that these two constructions are inverse to one another. Given a K3 surface $X$ with two line bundles $L_{1}$ and $L_{2}$ with intersection matrix (1), let $Y_{12}$ be the natural image of $X$ in $\mathbb{P}\left(\mathrm{H}^{0}\left(X, L_{1}\right)^{\vee}\right) \times \mathbb{P}\left(\mathrm{H}^{0}\left(X, L_{2}\right)^{\vee}\right)$ and let $Y_{1}$ and $Y_{2}$ be the projections onto the respective factors. 
On the other hand, construct the element $A \in \mathrm{H}^{0}\left(X, L_{1}\right) \otimes \mathrm{H}^{0}\left(X, L_{2}\right) \otimes(\operatorname{ker} \mu)^{\vee}$ from $\left(X, L_{1}, L_{2}\right)$ as above, and let $X_{12}, X_{1}$, and $X_{2}$ be the $\mathrm{K} 3$ surfaces constructed from $A$ in the usual way. We claim that $X_{12}=Y_{12}$ and $X_{i}=Y_{i}$ as sets and as varieties.

By the construction of $A$ from the kernel of $\mu$, we have $A(x, y, \cdot)=0$ for any point $(x, y) \in Y_{12}$, so $Y_{12} \subset X_{12}$ and $Y_{i} \subset X_{i}$. Now the quartic polynomial defining $X_{1}$ is not identically zero, because $A$ must have nonzero tensor rank. Therefore, $X_{1}$ and $Y_{1}$ are both given by quartic polynomials and must be the same variety, and similarly for $X_{12}$ and $Y_{12}$.

Conversely, given a nondegenerate $A \in V_{1} \otimes V_{2} \otimes V_{3}$, let $X$ be the K3 surface $X_{12}$ constructed from $A$, and let $L_{1}$ and $L_{2}$ be the line bundles on $X$. Then the vector spaces $V_{1}$ and $\mathrm{H}^{0}\left(X, L_{1}\right)$ are naturally isomorphic, as are $V_{2}$ and $\mathrm{H}^{0}\left(X, L_{2}\right)$, and $V_{3}^{\vee}$ may be identified with the kernel of the multiplication map $\mu$ in (3). With these identifications, the element of $\mathrm{H}^{0}\left(X, L_{1}\right) \otimes \mathrm{H}^{0}\left(X, L_{2}\right) \otimes(\operatorname{ker} \mu)^{\vee}$ constructed from this geometric data is well defined and $G$-equivalent to the original $A$.

REMARK 4.8. Strictly speaking, we have not shown that, for a generic point of the moduli space $\mathcal{M}=\mathcal{M}_{\mathfrak{O}_{20}}$ of $\mathrm{K} 3$ surfaces lattice-polarized by the 2dimensional lattice $\mathfrak{O}_{20}$ with matrix

$$
\left(\begin{array}{ll}
4 & 6 \\
6 & 4
\end{array}\right)
$$

the two line bundles $L_{1}$ and $L_{2}$ give quartic embeddings-we have only showed this for K3 surfaces lattice-polarized by $\langle 4\rangle$. Let $B \subset \mathcal{M}_{4}$ be the divisor in $\mathcal{M}_{4}$ corresponding to $\mathrm{K} 3$ surfaces for which the polarization is the class of a hyperelliptic curve; it is 18-dimensional. There are two obvious maps $\phi_{i}: \mathcal{M} \rightarrow$ $\mathcal{M}_{4}$, taking $\left(X, L_{1}, L_{2}\right)$ to $\left(X, L_{i}\right)$. For any value of $i \in\{0,1\}$, since $\mathcal{M}$ is 18 dimensional, in principle it is possible that the 'bad' subvariety $\phi_{i}^{-1}(B)$ of $\mathcal{M}$ for which the polarization $L_{i}$ gives a hyperelliptic curve coincides with all of $\mathcal{M}$. However, this does not happen, and there are at least two ways to see why. First, one may see it directly in this special example, as follows. Suppose $\left|L_{1}\right|$ gives a 2-to-1 map $\phi$ to a quadric surface. Then we have $L_{1}=E+F$, where $E$ and $F$ are pullbacks of the generators of the Picard group of the quadric surface. They satisfy $E^{2}=F^{2}=0$ and $E \cdot F=2$. However, the original 2-dimensional lattice has no isotropic vectors, which implies that the locus of 'bad' $\mathrm{K} 3$ surfaces is a Noether-Lefschetz divisor in $\mathcal{M}$.

Another more general way to see that generically $L_{1}$ and $L_{2}$ should give quartic embeddings is the following: the locus $Z$ of $\mathrm{K} 3$ surfaces for which the corresponding map is 2-to-1 to a quadric surface, or is composed with a pencil, is closed in the moduli space $\mathcal{M}$. Therefore, it suffices to show that the moduli space 
is irreducible, and to show that it contains a point outside $Z$. The first assertion follows (over $\mathbb{C}$ ) from the description as a quotient of a Hermitian symmetric domain, and the second from the 'forward' construction which produces such a K3 surface from a $4 \times 4 \times 4$ cube. We will use this more general method, without further mention, in the doubly and triply symmetrized cases of Rubik's revenge. The irreducibility follows from the uniqueness of the embedding of $\Lambda$ into the K 3 lattice.

4.4. Automorphisms. Next, let us compute the action of $\Phi^{*}=\left(\psi_{31} \circ \psi_{23} \circ\right.$ $\left.\psi_{12}\right)^{*}$ on the part of the Néron-Severi lattice given by (1) for K3 surfaces associated to generic orbits. The relation (2) holds also for the analogous divisors on $X_{2}$ and $X_{3}$, and in the generic case, there are no singularities. We therefore have

$$
\begin{aligned}
3 H & =C+D \\
3 C & =\Phi^{*}(D)+H \\
3 \Phi^{*}(D) & =\Phi^{*}(H)+C \\
3 \Phi^{*}(H) & =\Phi^{*}(C)+\Phi^{*}(D),
\end{aligned}
$$

where each relation is the analogue of (2) for $X_{1}, X_{2}, X_{3}$, and then $X_{1}$ again, when applying the $\psi_{i j}$ in $\Phi$ in turn. Thus, the automorphism $\Phi^{*}$ acts on the sublattice $N_{0}:=\mathbb{Z} H+\mathbb{Z} C \cong \mathfrak{O}_{20}$ of $\mathrm{NS}\left(X_{1}\right)$ by the matrix

$$
M=\left(\begin{array}{cc}
-3 & 8 \\
-8 & 21
\end{array}\right)
$$

in the basis $(H, C)$. It describes an automorphism of infinite order, and in fact

$$
M^{n}=\left(\begin{array}{cc}
-F_{6 n-2} & F_{6 n} \\
-F_{6 n} & F_{6 n+2}
\end{array}\right)
$$

where the $F_{n}$ denote the Fibonacci numbers $F_{0}=0, F_{1}=1, F_{n}=F_{n-1}+F_{n-2}$. The group generated by $M$ has index 6 in the integral orthogonal group $O\left(N_{0}, \mathbb{Z}\right)$ of $N_{0}$. For a very general such $X$ (that is, if $\operatorname{NS}(X)=N_{0}$ ), it can be shown that $\Phi$ generates $\operatorname{Aut}(X)$.

Note that the automorphism $\Phi$ of $X$ is the same as the automorphism considered by Cayley [23, Section 69], and more recently in the context of dynamics on K3 surfaces by Oguiso [61], who showed that for those $X$ having Picard number 2, the automorphism $\Phi$ is fixed-point-free and has positive entropy (see also [34] for more on this case). In Section 17.1, we will give a simple proof of this theorem, as well as of various extensions, using hyperdeterminants. 


\section{Doubly symmetric Rubik's revenge: $4 \otimes \operatorname{Sym}^{2}(4)$}

We now consider doubly symmetric $4 \times 4 \times 4$ cubical matrices, namely elements of the space $V_{1} \otimes \operatorname{Sym}^{2} V_{2}$ for 4-dimensional $F$-vector spaces $V_{1}$ and $V_{2}$. Since the natural injection of $V_{1} \otimes \operatorname{Sym}^{2} V_{2}$ into $V_{1} \otimes V_{2} \otimes V_{2}$ is equivariant for the $\mathrm{GL}\left(V_{1}\right) \times \mathrm{GL}\left(V_{2}\right)$-actions, one can understand the $\mathrm{GL}\left(V_{1}\right) \times \mathrm{GL}\left(V_{2}\right)$-orbits of $V_{1} \otimes \operatorname{Sym}^{2} V_{2}$ using Theorem 4.1.

However, there are some important differences in the geometric data attached to a general $4 \times 4 \times 4$ cube compared to that attached to a symmetric one. For a general $4 \times 4 \times 4$ cube, the three resulting K3 surfaces are nonsingular. The basic reason is that in the $\mathbb{P}^{15}$ of $4 \times 4$ matrices, the variety of matrices having rank at most two is 11-dimensional and thus will not intersect a general $\mathbb{P}^{3} \subset \mathbb{P}^{15}$ spanned by four $4 \times 4$ matrices. As a result, the corresponding determinantal quartic surface will have no rank singularities and will in fact generically be smooth.

In the $\mathbb{P}^{9}$ of symmetric $4 \times 4$ matrices, the matrices having rank at most two form a 6-dimensional variety of degree 10 , namely, the secant variety to the image of the Veronese embedding $\mathbb{P}^{3} \hookrightarrow \mathbb{P}^{9}$. A general $\mathbb{P}^{3} \subset \mathbb{P}^{9}$ spanned by four $4 \times 4$ matrices will intersect the variety of matrices of rank $\leqslant 2$ in a 0 -dimensional subscheme of degree 10; consequently, our determinantal quartic surface will have 10 isolated rank singularities, which are in fact nodes, and generically, there will be no other singularities.

These K 3 surfaces, cut out by determinants of a symmetric $4 \times 4$ matrix of linear forms, were also classically studied, and are called quartic symmetroids $[23,26$, 41]. We prove that the general orbits of tensors in $V_{1} \otimes \operatorname{Sym}^{2} V_{2}$ correspond to certain K3 surfaces with Picard rank at least 11 over $\bar{F}$ :

THEOREM 5.1. Let $V_{1}$ and $V_{2}$ be 4-dimensional vector spaces over $F$. Let $G^{\prime}=$ $\operatorname{GL}\left(V_{1}\right) \times \operatorname{GL}\left(V_{2}\right)$, and let $G$ be the quotient of $G^{\prime}$ by the kernel of the natural multiplication map on scalars $\mathbb{G}_{m} \times \mathbb{G}_{m} \rightarrow \mathbb{G}_{m}$ sending $\left(\gamma_{1}, \gamma_{2}\right)$ to $\gamma_{1} \gamma_{2}^{2}$. Let $V$ be the space $V_{1} \otimes \operatorname{Sym}^{2} V_{2}$. Let $\Lambda$ be the lattice given by the Gram matrix

$$
\left(\begin{array}{ccccccccccc}
4 & 6 & 0 & 0 & 0 & 0 & 0 & 0 & 0 & 0 & 0 \\
6 & 4 & 1 & 1 & 1 & 1 & 1 & 1 & 1 & 1 & 1 \\
0 & 1 & -2 & 0 & 0 & 0 & 0 & 0 & 0 & 0 & 0 \\
0 & 1 & 0 & -2 & 0 & 0 & 0 & 0 & 0 & 0 & 0 \\
0 & 1 & 0 & 0 & -2 & 0 & 0 & 0 & 0 & 0 & 0 \\
0 & 1 & 0 & 0 & 0 & -2 & 0 & 0 & 0 & 0 & 0 \\
0 & 1 & 0 & 0 & 0 & 0 & -2 & 0 & 0 & 0 & 0 \\
0 & 1 & 0 & 0 & 0 & 0 & 0 & -2 & 0 & 0 & 0 \\
0 & 1 & 0 & 0 & 0 & 0 & 0 & 0 & -2 & 0 & 0 \\
0 & 1 & 0 & 0 & 0 & 0 & 0 & 0 & 0 & -2 & 0 \\
0 & 1 & 0 & 0 & 0 & 0 & 0 & 0 & 0 & 0 & -2
\end{array}\right)
$$


and let $S=\left\{e_{1}, e_{2}\right\}$. Then the $G(F)$-orbits of an open subset of $V(F)$ are in bijection with the F-points of an open subvariety of the moduli space $\mathcal{M}_{\Lambda, S}$ of nonsingular $K 3$ surfaces lattice-polarized by $(\Lambda, S)$.

For a generic $A \in V_{1} \otimes \operatorname{Sym}^{2} V_{2}$, from the constructions in the previous section, we obtain nonsingular quartic surfaces $X_{2}$ and $X_{3}$ by slicing the cube $A$ in two directions, whereas in the third direction, we get a quartic surface $X_{1}$ with generically ten $A_{1}$ singularities. By symmetry, $X_{2}$ and $X_{3}$ are in fact identical surfaces in $\mathbb{P}\left(V_{2}^{\vee}\right)$, but we will sometimes refer to these separately in the sequel.

Because contracting the cube in the third direction gives a symmetric matrix, we see that for a generic point $x \in X_{1}$, the left and right kernels of $\left.A\right\lrcorner x$ are spanned by the same vector. The map $\psi_{12}: X_{1} \rightarrow X_{2}$ has a base locus consisting of the ten singularities on $X_{1}$ (since the kernel of $\left.A\right\lrcorner x$ at these ten points is (generically) 2-dimensional), and hence it is the minimal resolution of these singularities. Similarly, the map $\psi_{21}: X_{2} \rightarrow X_{1}$ is the blow-down of the exceptional divisors, so it is just the map $\psi_{12}^{-1}$ as a rational map. Furthermore, while $\psi_{13} \circ \psi_{21}$ is the identity map from $X_{2}$ to $X_{3}$, the maps $\psi_{23}$ and $\psi_{32}$ are not the identity map.

5.1. Néron-Severi lattice. We begin by describing a set of generators for the Néron-Severi group for the K3 surface $X$ arising from a very general doubly symmetric $4 \times 4 \times 4$ cubical matrix. Let $L_{1}$ be the hyperplane class of $X=X_{1}$ and $L_{2}$ be the pullback of the hyperplane class of $X_{2}$ via $\psi_{12}$. Finally, let $P_{1}, \ldots$, $P_{10}$ be the exceptional divisors corresponding to the ten singular points. While $L_{1}$, $L_{2}$, and $\sum_{i=1}^{10} P_{i}$ are defined over $F$, the $P_{i}$ individually may not be.

Proposition 5.2. The Picard group of $X_{\bar{F}}$ is generated by $L_{1}, L_{2}$ and the classes of the $P_{i}$.

Proof. We first observe that the dimension of the moduli space of quartic symmetroids is $10 \cdot 4-15-15-1=9$. Hence the Picard number is at most 11 . Since the classes of $L_{1}$ and the ten $P_{i}$ are all independent, the Picard number is exactly 11 for a very general point on the moduli space, and there are exactly ten singular points on the associated quartic surface. We obtain the relation

$$
3 L_{1}=2 L_{2}+\sum P_{i}
$$

by specializing the relation (2). Hence a basis for the span of all these classes is given by $\left\{L_{1}, L_{2}, P_{1}, \ldots, P_{9}\right\}$. We easily compute that the discriminant of the lattice $\Lambda$ they span is $1024=2^{10}$ and the discriminant group is $\mathbb{Z} / 4 \mathbb{Z} \oplus(\mathbb{Z} / 2 \mathbb{Z})^{8}$. 
It is enough to show that $\Lambda$ is saturated in $\overline{\mathrm{NS}}(X)$. In fact, by computing the inverse of the Gram matrix, one immediately checks that any element of the dual lattice of $\Lambda$ must have the form

$$
D=\frac{c}{4} L_{1}+\frac{1}{2}\left(\sum_{i=1}^{9} d_{i} P_{i}\right),
$$

for integers $c$ and $d_{i}$. Suppose $D$ is in the saturation $\Lambda^{\prime}$ of $\Lambda$. If $c$ is odd, then $2 D-\sum d_{i} P_{i}=(c / 2) L_{1}$ is also in $\Lambda^{\prime}$, which is a contradiction since its selfintersection is odd. Therefore, we may assume that $D$ has the form

$$
D=\frac{c}{2} L_{1}+\frac{1}{2}\left(\sum_{i=1}^{9} d_{i} P_{i}\right) .
$$

By symmetry, it follows that $(c / 2) L_{1}+\frac{1}{2}\left(\sum_{i=1}^{8} d_{i} P_{i}\right)+d_{9} P_{10} \in \overline{\mathrm{NS}}(X)$, and therefore we have $\frac{1}{2} d_{9}\left(P_{9}-P_{10}\right) \in \overline{\mathrm{NS}}(X)$. By Lemma 2.3, this forces $d_{9}$ to be even. Similarly, all the $d_{i}$ are even, and then $(c / 2) L_{1} \in \overline{\mathrm{NS}}(X)$, which is a contradiction as above. This concludes the proof.

\subsection{Moduli problem.}

Proof 1 of Theorem 5.1. As before, one direction has already been proved. Starting with a K3 surface $X$ and line bundles $L_{1}, L_{2}$ and $P_{1}, \ldots, P_{10}$ satisfying the desired intersection relations, we need to construct a doubly symmetric $4 \times 4 \times 4$ matrix, or equivalently, a symmetric $4 \times 4$ matrix of linear forms. This construction is described in, for example, [31, Section 4.2] (see also [26, 71]); we briefly sketch the argument:

Assume without loss of generality that $L_{1}$ and $L_{2}$ are very ample. Let $Y$ be the image of the quartic embedding corresponding to the line bundle $L_{1}$. For each $i$, since $P_{i}^{2}=-2$ and $P_{i} \cdot L_{2}>0$, we have that $P_{i}$ is effective and thus corresponds to a smooth rational curve on $X$. These collapse to singular points on $Y$, since $L_{1}$. $P_{i}=0$. The surface $Y$ has ten singular points. Next, let $\mathcal{F}$ be the pushforward of $L_{2}$ from $X$ to $Y$. We compute that $\mathcal{F}^{\vee}$ has the divisor class $-L_{2}-\sum P_{i}$. Therefore, the relation

$$
\mathcal{F} \cong \mathcal{F}^{\vee}
$$

holds in the Picard group of $Y$, so the ACM sheaf $\mathcal{F}$ gives a symmetric determinantal representation.

Checking that these constructions are inverse to one another is a similar verification as in the proof of Theorem 4.1. 
We may give a second, more elementary proof of Theorem 5.1, using the construction in the proof of Theorem 4.1 together with the following lemma:

LEMma 5.3. Let $B$ and $C$ be two $n \times n$ matrices over $F$ with $C$ invertible. Assume $B C^{-1}$ has distinct eigenvalues over $\bar{F}$ and that for all $x, y \in \bar{F}$, the transpose of the left kernel of $B x-C y$ is equal to its right kernel. Then $B$ and $C$ are symmetric matrices.

Proof. Since $\operatorname{det}\left(B C^{-1}-\lambda I\right)$ has distinct roots in $\bar{F}$ by assumption, the binary $n$-ic form $\operatorname{det}(B x-C y)=\operatorname{det}(C) \operatorname{det}\left(B C^{-1} x-I y\right)$ has distinct roots $\left[x_{i}: y_{i}\right]$ $(i=1, \ldots, n)$ in $\mathbb{P}^{1}(\bar{F})$. For each $i$, let $v_{i}$ be a nonzero vector in the right kernel of $B x_{i}-C y_{i}$, implying that $v_{i}^{t}$ is a nonzero vector in the left kernel. The vectors $v_{i}$ are linearly independent, because they are eigenvectors corresponding to the distinct eigenvalues of $B C^{-1}$.

Consider the two symmetric bilinear forms $B(\cdot, \cdot)$ and $C(\cdot, \cdot)$ defined by $B(w, z)=w^{t} B z$ and $C(w, z)=w^{t} C z$. We wish to show that $B$ and $C$ are symmetric bilinear forms. To see this, note that $v_{i}^{t}\left(B x_{i}-C y_{i}\right) v_{j}=v_{i}^{t}\left(B x_{j}-\right.$ $\left.C y_{j}\right) v_{j}=0$ for any $i \neq j$. Since $\left(x_{i}, y_{i}\right)$ and $\left(x_{j}, y_{j}\right)$ are linearly independent (as they yield distinct points in $\mathbb{P}^{1}(\bar{F})$ ), we conclude that $v_{i}^{t} B v_{j}=v_{i}^{t} C v_{j}=0$ for any $i \neq j$.

It follows that $B$ and $C$ are diagonal bilinear forms with respect to the basis $v_{1}, \ldots, v_{n}$. Hence $B$ and $C$ are symmetric bilinear forms, and thus correspond to symmetric matrices with respect to any basis.

Proof 2 of Theorem 5.1. Again, we only need to show that the geometric data gives rise to a doubly symmetric $4 \times 4 \times 4$ matrix. Given $\left(X, L_{1}, L_{2}, P_{1}, \ldots\right.$, $P_{10}$ ), we use the multiplication map

$$
\mu: \mathrm{H}^{0}\left(X, L_{1}\right) \otimes \mathrm{H}^{0}\left(X, L_{2}\right) \rightarrow \mathrm{H}^{0}\left(X, L_{1} \otimes L_{2}\right)
$$

to obtain a $4 \times 4 \times 4$ matrix $A$ as before. It remains to show that there exists an identification of $V_{3}:=(\operatorname{ker} \mu)^{\vee}$ and $V_{2}:=\mathrm{H}^{0}\left(X, L_{2}\right)$ such that $A$ is doubly symmetric. Let $V_{1}:=\mathrm{H}^{0}\left(X, L_{1}\right)$.

The proof of Theorem 4.1 implies that $A$ in turn produces a K3 surface and line bundles isomorphic to those with which we started. In particular, the embedding corresponding to $L_{1}$ has ten singular points, since $L_{1} \cdot P_{i}=0$ implies that these (-2)-curves are contracted. Therefore, by applying equation (2) of Section 4.2 and comparing with the relation (4), we deduce that the line bundles $L_{2}$ and $L_{3}$ are isomorphic. We therefore have an isomorphism $\phi: V_{2} \stackrel{\sim}{\rightarrow} V_{3}$. Let $X_{1}$ be the image of $X$ via the quartic embedding given by $L_{1}$. For any point $x \in X_{1}$, we have $\operatorname{det}(A(x, \cdot, \cdot))=0$ and the kernel of $A(x, \cdot, \cdot)$ in $V_{2}^{\vee}$ and in $V_{3}^{\vee}$ is the same 
(under $\phi$ ). In other words, since $X_{1}$ spans $\mathbb{P}\left(V_{1}^{\vee}\right)$, the image of $V_{1}^{\vee}$ in $V_{2} \otimes V_{2}$ given by $(\operatorname{Id} \otimes \phi) \circ A$ is a 4-dimensional subspace of $V_{2} \otimes V_{2}$ such that the 'left' and 'right' kernels of each element in $V_{2}^{\vee}$ are the same (usually empty, of course).

We now wish to apply Lemma 5.3 to any two generic matrices in this 4dimensional space. For two nonsingular elements $B$ and $C$ of $V_{2} \otimes V_{2}$, the matrix $B C^{-1}$ will have distinct eigenvalues over $\bar{F}$ if the binary $n$-ic form $\operatorname{det}(B x-C y)$ has distinct roots, in which case $B x-C y$ has rank at least 3 for any values of $x$ and $y$. Recall that the K3 surface $X_{1}$ has only a finite number of isolated singularities, points $x \in \mathbb{P}\left(V_{1}^{\vee}\right)$ where $A(x, \cdot, \cdot)$ has rank 2 . For any line in $\mathbb{P}\left(V_{1}^{\vee}\right)$ not passing through one of those singularities, the corresponding pencil of matrices in $\mathbb{P}\left(V_{2} \otimes V_{2}\right)$ will thus satisfy the conditions of the lemma. That is, let $B$ and $C$ be nonsingular elements of $V_{2} \otimes V_{2}$ such that their span does not contain an element with rank less than 3. Lemma 5.3 implies that $B$ and $C$ are symmetric. We may repeat this process to obtain a basis for the image of $V_{1}^{\vee}$ in $V_{2} \otimes V_{2}$ only consisting of symmetric elements, thereby giving an element of $V_{1} \otimes \operatorname{Sym}^{2} V_{2}$ as desired.

Since these constructions are inverse to one another in the proof of Theorem 4.1, they are also inverse to one another here.

5.3. Automorphisms. The map $\Phi=\psi_{31} \circ \psi_{23} \circ \psi_{12}: X_{1} \rightarrow X_{1}$ considered in Section 4.4 can be constructed in this situation as well. Though it is only a birational automorphism of $X_{1}$, it can be lifted to an actual automorphism of the blown-up nonsingular model $X_{12}$. This follows from the general fact that a birational map between two minimal nonsingular algebraic surfaces with nonnegative Kodaira dimension is an isomorphism (see, for instance, [3, Theorem 10.21]).

First, we observe that $\Phi$ is an involution. Indeed, the symmetry implies that $\psi_{12}=\psi_{13}, \psi_{23}=\psi_{32}$, and $\psi_{31}=\psi_{21}$, and thus $\Phi=\Phi^{-1}$. We now compute its induced action on the Néron-Severi group.

The main idea is the same as in Section 4.4: use the relation (2) repeatedly, as we apply the maps $\psi_{12}, \psi_{23}, \psi_{31}$, and $\psi_{12}$ again. Let $L_{1}, L_{2}$, and $P_{i}$ for $1 \leqslant i \leqslant 10$ be the classes introduced earlier. Then we obtain the following equations among these classes (written additively):

$$
\begin{aligned}
3 L_{1} & =2 L_{2}+\sum_{i} P_{i}, & 3 L_{2} & =L_{1}+L_{2}, \\
3 \Phi^{*}\left(L_{2}\right) & =\Phi^{*}\left(L_{1}\right)+L_{2}, & 3 \Phi^{*}\left(L_{1}\right) & =2 \Phi^{*}\left(L_{2}\right)+\sum_{i} \Phi^{*}\left(P_{i}\right) .
\end{aligned}
$$

By checking intersection numbers, we compute that $\Phi^{*} L_{1}=-3 L_{1}+8 L_{2}$, $\Phi^{*} L_{2}=-L_{1}+3 L_{2}$, and $\Phi^{*} P_{i}=2 L_{1}-\sum_{j \neq i} P_{j}$. The associated transformation matrix squares to the identity, as expected. 
REMARK 5.4. The automorphism group of a general quartic symmetroid contains a subgroup of the automorphism group of a general nodal Enriques surface. The latter group is a finite-index subgroup of the reflection group $W_{2,4,6}$ corresponding to the Coxeter diagram of type $T_{2,4,6}$, and was computed explicitly by Cossec and Dolgachev [25].

\section{Triply symmetric Rubik's revenge: $\operatorname{Sym}^{3}(4)$}

We consider next the triply symmetric Rubik's revenge, in order to understand the orbits of $\mathbb{G}_{m} \times \mathrm{GL}(V)$ on $\operatorname{Sym}^{3} V$ for a 4-dimensional vector space $V$.

Such a cube is doubly symmetric in all three directions, and the three K3's arising from such a triply symmetric Rubik's revenge are identical. A generic such triply symmetric Rubik's revenge will thus give rise to a $\mathrm{K} 3$ that has at least 10 singularities, and a numerical example shows that we obtain exactly 10 singularities in general.

The quartic surface $X$ has been well studied in the classical literature $[23,38$, $39,41]$, as a Hessian quartic symmetroid, since the matrix of linear forms whose determinant defines $X$ is the Hessian (the matrix of second partial derivatives) of a single cubic form $F$ in four variables. For more recent references, see [26, 32].

Generically, over an algebraically closed field, there are five planes tangent (along a degenerate conic) to such a Hessian surface. If $\ell_{i}$ are the linear forms defining these planes $Z_{i}$, the equation of the quartic may be written as

$$
\frac{1}{a_{1} \ell_{1}}+\cdots+\frac{1}{a_{5} \ell_{5}}=0
$$

for some constants $a_{1}, \ldots, a_{5}$. The cubic form is $F=a_{1} \ell_{1}^{3}+\cdots+a_{5} \ell_{5}^{3}$. The ten singular points are given by the intersections of all ten triples of the hyperplanes $Z_{i}$. In addition, the surface contains ten special lines, which come from the pairwise intersections of the $Z_{i}$. Thus, the singular points may be labeled $P_{i j k}$ and the lines $L_{l m}$, with $P_{i j k}$ lying on $L_{l m}$ exactly when $\{l, m\} \subset\{i, j, k\}$. Therefore, there are three singular points on each special line and three special lines passing through each singular point. For $1 \leqslant i \neq j \leqslant 3$, the maps $\psi_{i j}$ defined in Section 4 are all identical. Denoting them by $\psi$, it is clear that $\psi$ is a birational involution on the K3 surface $X$, blowing up the ten singular points $P_{i j k}$ and blowing down the $L_{l m}$. In fact, it exchanges $P_{i j k}$ and $L_{l m}$, where $\{i, j, k, l, m\}=\{1,2,3,4,5\}$.

We show that the general orbits of tensors in $\operatorname{Sym}^{3} V$ correspond to certain K3 surfaces with Picard rank at least 16 over $\bar{F}$.

THEOREM 6.1. Let $V_{1}$ be a 4-dimensional vector space over $F$. Let $G^{\prime}=\mathbb{G}_{m} \times$ $\mathrm{GL}\left(V_{1}\right)$ and $G$ be the quotient of $G^{\prime}$ by the kernel of the multiplication map $\mathbb{G}_{m} \times$ 
$\mathbb{G}_{m} \rightarrow \mathbb{G}_{m}$ given by $\left(\lambda_{1}, \lambda_{2}\right) \mapsto \lambda_{1} \lambda_{2}^{3}$. Let $V$ be the representation $\mathrm{Sym}^{3} V_{1}$ of $G$. Let $\Lambda$ be the lattice given by the Gram matrix

$$
\left(\begin{array}{cccccccccccccccc}
4 & 6 & 1 & 1 & 1 & 1 & 1 & 0 & 0 & 0 & 0 & 0 & 0 & 0 & 0 & 0 \\
6 & 4 & 0 & 0 & 0 & 0 & 0 & 1 & 1 & 1 & 1 & 1 & 1 & 1 & 1 & 1 \\
1 & 0 & -2 & 0 & 0 & 0 & 0 & 1 & 1 & 1 & 0 & 0 & 0 & 0 & 0 & 0 \\
1 & 0 & 0 & -2 & 0 & 0 & 0 & 1 & 0 & 0 & 1 & 1 & 0 & 0 & 0 & 0 \\
1 & 0 & 0 & 0 & -2 & 0 & 0 & 0 & 1 & 0 & 1 & 0 & 1 & 0 & 0 & 0 \\
1 & 0 & 0 & 0 & 0 & -2 & 0 & 1 & 0 & 0 & 0 & 0 & 0 & 1 & 1 & 0 \\
1 & 0 & 0 & 0 & 0 & 0 & -2 & 0 & 0 & 0 & 1 & 0 & 0 & 1 & 0 & 0 \\
0 & 1 & 1 & 1 & 0 & 1 & 0 & -2 & 0 & 0 & 0 & 0 & 0 & 0 & 0 & 0 \\
0 & 1 & 1 & 0 & 1 & 0 & 0 & 0 & -2 & 0 & 0 & 0 & 0 & 0 & 0 & 0 \\
0 & 1 & 1 & 0 & 0 & 0 & 0 & 0 & 0 & -2 & 0 & 0 & 0 & 0 & 0 & 0 \\
0 & 1 & 0 & 1 & 1 & 0 & 1 & 0 & 0 & 0 & -2 & 0 & 0 & 0 & 0 & 0 \\
0 & 1 & 0 & 1 & 0 & 0 & 0 & 0 & 0 & 0 & 0 & -2 & 0 & 0 & 0 & 0 \\
0 & 1 & 0 & 0 & 1 & 0 & 0 & 0 & 0 & 0 & 0 & 0 & -2 & 0 & 0 & 0 \\
0 & 1 & 0 & 0 & 0 & 1 & 1 & 0 & 0 & 0 & 0 & 0 & 0 & -2 & 0 & 0 \\
0 & 1 & 0 & 0 & 0 & 1 & 0 & 0 & 0 & 0 & 0 & 0 & 0 & 0 & -2 & 0 \\
0 & 1 & 0 & 0 & 0 & 0 & 0 & 0 & 0 & 0 & 0 & 0 & 0 & 0 & 0 & -2
\end{array}\right)
$$

and let $S=\left\{e_{1}, e_{2}\right\}$. Then the $G(F)$-orbits of an open subset of $V(F)$ are in bijection with the F-points of an open subvariety of the moduli space $\mathcal{M}_{\Lambda, S}$ of nonsingular K3 surfaces lattice-polarized by $(\Lambda, S)$.

6.1. Néron-Severi lattice. The Picard group of the generic Hessian surface (base changed to $\bar{F}$ ) is spanned over $\mathbb{Z}$ by the classes of the lines $L_{i j}$ and the exceptional curves corresponding to the singular points $P_{i j k}$. The lattice spanned by these has rank 16 and discriminant -48 . Since its discriminant group is $\mathbb{Z} / 3 \mathbb{Z} \oplus(\mathbb{Z} / 2 \mathbb{Z})^{4}$, a case-by-case argument shows that this lattice is the full Picard group. We omit this proof, since the result is established in [32] (using elliptic fibrations) and by a different method in [27].

6.2. Moduli problem. We now proceed to the proof of Theorem 6.1. Given a triply symmetric $4 \times 4 \times 4$ cube and the resulting K3 surface $X$, we have seen that the Picard group of $X_{\bar{F}}$ is generated by the classes of the ten nodes and lines. Let $H_{1}$ and $H_{2}$ be the hyperplane classes for $X_{1}$ and $X_{2}$, respectively. The set $\left\{H_{1}, H_{2}, L_{12}, L_{13}, L_{14}, L_{23}, L_{34}, P_{123}, P_{124}, P_{125}, P_{134}, P_{135}, P_{145}, P_{234}, P_{235}, P_{245}\right\}$ is easily checked to be a basis for $\overline{\mathrm{NS}}(X)$, yielding the Gram matrix (5). This data is fixed up to isomorphism by the action of $\mathbb{G}_{m} \times \mathrm{GL}\left(V_{1}\right)$. 
Conversely, given a $(\Lambda, S)$-polarized $\mathrm{K} 3$ surface, we construct a triply symmetric Rubik's revenge by using the second proof of Theorem 5.1. In particular, we may use that proof to construct a $4 \times 4 \times 4$ cube $A$ in $V_{1} \otimes V_{2} \otimes V_{3}$, for certain 4-dimensional vector spaces $V_{i}$, where there is an isomorphism $\phi_{32}: V_{3} \rightarrow V_{2}$ so that $A$ is symmetric, that is, maps to an element of $V_{1} \otimes \operatorname{Sym}^{2} V_{2}$ under Id $\otimes \mathrm{Id} \otimes \phi_{32}$. Here, we may also use the same proof to show that $A$ is symmetric under an isomorphism $\phi_{21}: V_{2} \rightarrow V_{1}$, that is, gives an element of $\mathrm{Sym}^{2} V_{1} \otimes V_{3}$ under the map Id $\otimes \phi_{21} \otimes$ Id. Thus, since the transpositions (12) and (23) generate $S_{3}$, we may use $\phi_{12}$ and $\phi_{23}$ to identify all three vector spaces and obtain an element of $\operatorname{Sym}^{3} V_{1}$.

6.3. Automorphisms. The automorphism group of the Hessian quartic surface is quite large. In [32], Dolgachev and Keum identified a set of generators for the automorphism group. However, the relations between these are not completely known, so a complete presentation for the automorphism group is still unknown.

To connect with the earlier sections, we note that the maps $\psi$ are birational involutions (recall that they all are identical). In this case, each $\psi$ is also the same as the 3-cycle $\Phi$ described in Section 4.4 because of the symmetry. We described above the action induced by this involution on $\overline{\mathrm{NS}}(X)$ : the divisors $H_{1}$ and $H_{2}$ are switched, and the $P_{i j k}$ and $L_{l m}$ are switched for $\{i, j, k, l, m\}=\{1,2,3,4,5\}$. Again, this is studied extensively in [32].

\section{Penteracts (or 5-cubes): $2 \otimes 2 \otimes 2 \otimes 2 \otimes 2$}

Consider the representation $V=V_{1} \otimes V_{2} \otimes V_{3} \otimes V_{4} \otimes V_{5}$, where each $V_{n}$ is a 2-dimensional $F$-vector space, of the group $G^{\prime}=\operatorname{GL}\left(V_{1}\right) \times \operatorname{GL}\left(V_{2}\right) \times \operatorname{GL}\left(V_{3}\right) \times$ $\operatorname{GL}\left(V_{4}\right) \times \operatorname{GL}\left(V_{5}\right)$. With a choice of bases for each $V_{n}$ for $n \in\{1, \ldots, 5\}$, an element $A \in V(F)$ may be visualized as a 5-dimensional cube, or penteract, with entries $a_{i j k l m} \in F$ for $i, j, k, l, m \in\{1,2\}$. The space of penteracts is extremely rich, and indeed the next several sections, through Section 13, will focus on variations of this space of penteracts.

Let $G$ be the quotient of $G^{\prime}$ by the kernel of the multiplication map $\mathbb{G}_{m}^{5} \rightarrow \mathbb{G}_{m}$. In this section, we will study the $G(F)$-orbits on $V(F)$, and in particular, describe the relationship between (an open subvariety of) the orbit space $V(F) / G(F)$ and the moduli space of certain K3 surfaces having Néron-Severi rank at least 4:

THEOREM 7.1. Let $V=V_{1} \otimes V_{2} \otimes V_{3} \otimes V_{4} \otimes V_{5}$, where each $V_{n}$ is a 2-dimensional $F$-vector space. Let $G^{\prime}=\mathrm{GL}\left(V_{1}\right) \times \mathrm{GL}\left(V_{2}\right) \times \mathrm{GL}\left(V_{3}\right) \times \mathrm{GL}\left(V_{4}\right) \times \mathrm{GL}\left(V_{5}\right)$, and let $G$ be the quotient of $G^{\prime}$ by the kernel of the multiplication map $\mathbb{G}_{m}^{5} \rightarrow \mathbb{G}_{m}$. Let 
$\Lambda$ be the lattice whose Gram matrix is

$$
\left(\begin{array}{llll}
0 & 2 & 2 & 2 \\
2 & 0 & 2 & 2 \\
2 & 2 & 0 & 2 \\
2 & 2 & 2 & 0
\end{array}\right),
$$

and let $S=\left\{e_{1}, e_{2}, e_{3}, e_{4}\right\}$. Then the $G(F)$-orbits of an open subset of $V(F)$ are in bijection with the $F$-rational points of an open subvariety of the moduli space $\mathcal{M}_{\Lambda, S}$ of $K 3$ surfaces $X$ lattice-polarized by $(\Lambda, S)$.

7.1. Constructions of $\mathbf{K} 3$ surfaces. Given a general $A \in V(F)$, we construct a K3 surface with Picard number at least 4 as follows. First, let

$$
X_{123}:=\left\{(v, w, x) \in \mathbb{P}\left(V_{1}^{\vee}\right) \times \mathbb{P}\left(V_{2}^{\vee}\right) \times \mathbb{P}\left(V_{3}^{\vee}\right): \operatorname{det} A(v, w, x, \cdot, \cdot)=0\right\} .
$$

We similarly define $X_{i j k}$ for any subset $\{i, j, k\}$ in $\{1,2,3,4,5\}$ (where permutation of the indices does not change the variety). The equation defining each $X_{i j k}$ is a tridegree $(2,2,2)$ form in $\mathbb{P}^{1} \times \mathbb{P}^{1} \times \mathbb{P}^{1}$ and thus $X_{i j k}$ is generically a $\mathrm{K} 3$ surface; specifically, we only allow isolated rational double point singularities. Also, let

$X_{1234}:=\left\{(v, w, x, y) \in \mathbb{P}\left(V_{1}^{\vee}\right) \times \mathbb{P}\left(V_{2}^{\vee}\right) \times \mathbb{P}\left(V_{3}^{\vee}\right) \times \mathbb{P}\left(V_{4}^{\vee}\right): A(v, w, x, y, \cdot)=0\right\}$,

and define $X_{i j k l}$ for any subset $\{i, j, k, l\}$ in $\{1,2,3,4,5\}$ in the analogous way. This variety $X_{i j k l}$ is the intersection of two multidegree $(1,1,1,1)$ forms in $\mathbb{P}^{1} \times$ $\mathbb{P}^{1} \times \mathbb{P}^{1} \times \mathbb{P}^{1}$, which is also generically a K3 surface. In other words, we can view the $\mathrm{K} 3$ surface as the base locus of a pencil of divisors of type $(1,1,1,1)$ in $\left(\mathbb{P}^{1}\right)^{4}$. Note that the projections from $X_{i j k l}$ to $\mathbb{P}\left(V_{i}^{\vee}\right)$ are genus one fibrations.

For any permutation $\{i, j, k, l, m\}$ of $\{1,2,3,4,5\}$, there exists a projection $\pi: X_{i j k l} \rightarrow X_{i j k}$, which is an isomorphism for the generic $A \in V(F)$. The fiber of each point $(v, w, x) \in X_{i j k}$ is determined by the kernel of the singular map $A(v, w, x): V_{l}^{\vee} \rightarrow V_{m}$. If $A(v, w, x, \cdot, \cdot)$ is the zero matrix, then $X_{i j k}$ is singular at $(v, w, x)$, and we then call $(v, w, x)$ a rank singularity. For nonsingular points $(v, w, x)$ on $X_{i j k}$, the bilinear form $A(v, w, x, \cdot, \cdot)$ has a 1-dimensional kernel, and the fiber of $(v, w, x)$ under the map $\pi$ is a single point, given algebraically. In particular, we see that if $X_{i j k}$ is nonsingular, then it is isomorphic to $X_{i j k l}$.

More generally, if $X_{i j k}$ has an isolated rank singularity at $(v, w, x)$, then the fiber of $\pi$ at $(v, w, x)$ is the entire line $\left\{(v, w, x, y) \in \mathbb{P}\left(V_{i}^{\vee}\right) \times \mathbb{P}\left(V_{j}^{\vee}\right) \times \mathbb{P}\left(V_{k}^{\vee}\right) \times\right.$ $\left.\mathbb{P}\left(V_{l}^{\vee}\right): y \in \mathbb{P}\left(V_{l}^{\vee}\right)\right\}$. Since such rational double point singularities are blown up in one step, the surfaces $X_{i j k l}$ are nonsingular even when the $X_{i j k}$ have isolated rank singularities. We call a penteract nondegenerate if the surfaces $X_{i j k l}$ are all nonsingular. 
7.2. Néron-Severi lattice. We now compute the Néron-Severi lattice of $X=$ $X_{1234}$. The surface $X$ comes equipped with the four line bundles $L_{i}$, given by the pullbacks of $\mathcal{O}_{\mathbb{P}\left(V_{i}^{\vee}\right)}(1)$ for $1 \leqslant i \leqslant 4$. Their intersection numbers are easy to compute: because of the description of $X_{i j k}$ as the vanishing of a tridegree (2,2, 2) form, we have $L_{i} \cdot L_{j}=2\left(1-\delta_{i j}\right)$. As the dimension of the orbit space is $2^{5}-(3 \cdot 5+1)=16$, the dimension of $\mathrm{NS}(X)$ for a generic $X$ in this family must be exactly 4 . Therefore, although we will find other natural divisors, these four $L_{i}$ generate the Picard group of the generic K3 surface in this family.

There are other line bundles given by, for example, considering the pullback of $\mathcal{O}_{\mathbb{P}\left(V_{5}^{\vee}\right)}(1)$ via the isomorphisms $X=X_{1234} \rightarrow X_{123} \rightarrow X_{1235}$ followed by the projection to $\mathbb{P}\left(V_{5}^{\vee}\right)$. This particular line bundle $L_{5}^{(123)}$ satisfies the following:

LEMMA 7.2. If $X_{123}$ is nonsingular (and therefore isomorphic to $X$ ), then we have the relation

$$
L_{1}+L_{2}+L_{3}=L_{4}+L_{5}^{(123)}
$$

among the above line bundles on $X$. More generally, if $X_{123}$ has isolated rational double point singularities, we have

$$
L_{1}+L_{2}+L_{3}=L_{4}+L_{5}^{(123)}+\sum_{i} E_{i}
$$

where the $E_{i}$ are the line bundles corresponding to the exceptional divisors on $X$ arising from the singularities on $X_{123}$.

Proof. We first assume that $X_{123}$ is smooth. The rational maps $v_{4}$ and $v_{5}$ from $X_{123}$ to $\mathbb{P}\left(V_{4}^{\vee}\right)$ and $\mathbb{P}\left(V_{5}^{\vee}\right)$, which define $L_{4}$ and $L_{5}^{(123)}$, respectively, are each given by the appropriate kernel of $A(v, w, x, \cdot, \cdot)$ in $V_{4} \otimes V_{5}$. With a choice of basis vectors, let

$$
A(v, w, x, \cdot, \cdot)=\left(\begin{array}{ll}
A_{11} & A_{12} \\
A_{21} & A_{22}
\end{array}\right),
$$

where each $A_{i j}$ is a tridegree $(1,1,1)$ form on $\mathbb{P}\left(V_{1}^{\vee}\right) \times \mathbb{P}\left(V_{2}^{\vee}\right) \times \mathbb{P}\left(V_{3}^{\vee}\right)$. Then $v_{4}$ and $v_{5}$ are given by, for example, the forms $\left[-A_{21}: A_{11}\right]$ and $\left[-A_{12}: A_{11}\right]$, respectively. It is easy to check that the line bundle $v_{4}^{*} \mathcal{O}_{\mathbb{P}\left(V_{4}^{\vee}\right)}(1)$ is isomorphic to $\mathcal{O}\left(Z\left(A_{11}, A_{12}\right)\right)$, where $Z\left(A_{11}, A_{12}\right)$ refers to the common zero locus of those two forms; similarly, $v_{5}^{*} \mathcal{O}_{\mathbb{P}\left(V_{5}^{\vee}\right)}(1)$ is isomorphic to $\mathcal{O}\left(Z\left(A_{11}, A_{21}\right)\right)$. Thus, the right side of (7) is isomorphic to $\mathcal{O}\left(Z\left(A_{11}\right)\right)$, and thus to the pullback of $\mathcal{O}(1,1,1)$ from $\mathbb{P}\left(V_{1}^{\vee}\right) \times \mathbb{P}\left(V_{2}^{\vee}\right) \times \mathbb{P}\left(V_{3}^{\vee}\right)$ to $X$.

If $X_{123}$ has isolated rational double point singularities, then for the singular points $(v, w, x)$, we have that $A(v, w, x, \cdot, \cdot)$ is identically zero. Thus, the divisors $Z\left(A_{11}\right)$ on $X_{123}$ contains components corresponding to $L_{4}$, to $L_{5}^{(123)}$, and to each of the singularities, giving (8). 
We will use versions of the relation of Lemma 7.2 (with permuted indices, as necessary) to determine how divisor classes interact in many of the subsequent sections.

Proposition 7.3. For a very general $X$ in this family of $K 3$ surfaces, $\mathrm{NS}(X)$ is spanned over $\mathbb{Z}$ by $L_{1}, L_{2}, L_{3}$, and $L_{4}$.

Proof. Because this moduli space has dimension $32-16=16$, the Picard number of such a very general $X$ is at most 4 , and we know that $L_{1}, \ldots, L_{4}$ span a finiteindex subgroup of $\mathrm{NS}(X)$. The discriminant of the lattice generated by $L_{1}, L_{2}$, $L_{3}, L_{4}$ is $-48=-2^{4} \cdot 3$, and we only need to check that it is 2-saturated.

For $i \neq j$, the class $\left(L_{i}+L_{j}\right) / 2$ cannot be integral, since its self-intersection is odd, and similarly for $\left(L_{i}+L_{j}+L_{k}\right) / 2$ for $i, j, k$ distinct. By symmetry, therefore, $L_{i} / 2$ cannot be in $\mathrm{NS}(X)$ (otherwise $L_{i} / 2+L_{j} / 2$ would be). Finally, if $\left(L_{1}+L_{2}+L_{3}+L_{4}\right) / 2=L_{4}+L_{5}^{(123)} / 2$ were in $\mathrm{NS}(X)$, so would $L_{5}^{(123)} / 2$, and therefore all $L_{i} / 2$ by symmetry, which is a contradiction.

LEMMA 7.4. A K3 surface $X_{123}$ associated to a very general point in the moduli space of $(\Lambda, S)$-polarized $K 3$ surfaces has no linear automorphisms (that is, induced from $\mathrm{PGL}_{2} \times \mathrm{PGL}_{2} \times \mathrm{PGL}_{2}$ ) other than the identity.

Proof. We proceed as in the proof of Lemma 4.3. Let $g \in O^{+}(\mathrm{NS}(X))$ and $h \in$ $O_{\omega}(\mathrm{T}(X))$ agree on the discriminant groups. As before we can assume $h= \pm \mathrm{Id}$. On the other hand, $g$ fixes the classes of $L_{1}, L_{2}$ and $L_{3}$. Let

$$
g\left(L_{4}\right)=a L_{1}+b L_{2}+c L_{3}+d L_{4},
$$

for some integers $a, b, c, d$. Taking the intersection with $g\left(L_{1}\right)=L_{1}$ through $g\left(L_{3}\right)=L_{3}$ and using $g\left(L_{i}\right) \cdot g\left(L_{4}\right)=L_{i} \cdot L_{4}$, we obtain the equations

$$
\begin{aligned}
& 2=0+2 b+2 c+2 d \\
& 2=2 a+0+2 c+2 d \\
& 2=2 a+2 b+0+2 d,
\end{aligned}
$$

implying $a=b=c$. These equations reduce to $2=4 a+2 d$, or $d=1-2 a$. Finally, $g\left(L_{4}\right)^{2}=L_{4}^{2}=0$ yields $a(a+d)=0$. Now $a=0$ implies $d=1$ and $g=\mathrm{Id}$, whereas $a=-d$ implies $d=-1$. But in the latter case, $\bar{g}$ is not $\pm \mathrm{Id}$, a contradiction. This completes the proof.

COROLlary 7.5. The stabilizer of the action of $G$ on $V$ is generically trivial. 
Proof. If $g=\left(g_{1}, g_{2}, g_{3}, g_{4}, g_{5}\right)$ stabilizes $v \in V$, then each triple $\left(g_{i}, g_{j}\right.$, $g_{k}$ ) gives a linear automorphism of $X_{i j k}$ for each $(i, j, k)$. Therefore, generically $g=1$.

7.3. Reverse construction. We now give the proof of the reverse direction of Theorem 7.1. We start from the data of a nonsingular K3 surface $X$ with nonisomorphic line bundles $L_{1}, L_{2}, L_{3}$, and $L_{4}$ such that $L_{i} \cdot L_{j}=2\left(1-\delta_{i j}\right)$. It follows from Riemann-Roch that $L_{i}$ or $L_{i}^{-1}$ is effective, since $\mathrm{h}^{0}\left(L_{i}\right)+\mathrm{h}^{0}\left(L_{i}^{-1}\right) \geqslant$ 2. We assume the former without loss of generality, noting that $L_{i} \dot{L}_{j}=2$ forces a compatible choice. Consider the multiplication map

$\mu: \mathrm{H}^{0}\left(X, L_{1}\right) \otimes \mathrm{H}^{0}\left(X, L_{2}\right) \otimes \mathrm{H}^{0}\left(X, L_{3}\right) \otimes \mathrm{H}^{0}\left(X, L_{4}\right) \rightarrow \mathrm{H}^{0}\left(X, L_{1} \otimes L_{2} \otimes L_{3} \otimes L_{4}\right)$

on sections. The dimension of the domain is $2^{4}=16$. Since

$$
\left(L_{1}+L_{2}+L_{3}+L_{4}\right)^{2}=2 \cdot 2 \cdot 6=24,
$$

an easy application of Riemann-Roch on $X$ then yields

$h^{0}\left(X, L_{1}+L_{2}+L_{3}+L_{4}\right)=\frac{1}{2}\left(L_{1}+L_{2}+L_{3}+L_{4}\right)^{2}+\chi\left(\mathcal{O}_{X}\right)=\frac{24}{2}+2=14$.

Furthermore, we claim that the map (9) is surjective from repeated applications of the basepoint-free pencil trick [2, page 126]. We first check that a number of line bundles have vanishing $\mathrm{H}^{1}$ groups.

LEMMA 7.6. For generic $X$ and for distinct $i, j, k, \ell \in\{1,2,3,4\}$, the cohomology groups $\mathrm{H}^{1}\left(X, L_{i}^{-1}\right), \mathrm{H}^{1}\left(X, L_{i} \otimes L_{j}^{-1}\right), \mathrm{H}^{1}\left(X, L_{i} \otimes L_{j} \otimes L_{k}^{-1}\right)$, and $\mathrm{H}^{1}\left(X, L_{i} \otimes L_{j} \otimes L_{k} \otimes L_{\ell}^{-1}\right)$ all vanish.

Proof. By symmetry, it suffices to check that $\mathrm{H}^{1}\left(X, L_{1}^{-1}\right), \mathrm{H}^{1}\left(X, L_{1} \otimes L_{2}^{-1}\right)$, $\mathrm{H}^{1}\left(X, L_{1} \otimes L_{2} \otimes L_{3}^{-1}\right)$, and $\mathrm{H}^{1}\left(X, L_{1} \otimes L_{2} \otimes L_{3} \otimes L_{4}^{-1}\right)$ all vanish. Note that Riemann-Roch and Serre duality for each of these line bundles $L$ implies that

$$
\mathrm{h}^{1}(L)=\mathrm{h}^{0}(L)+\mathrm{h}^{0}\left(L^{-1}\right)-2-(L \cdot L) / 2 .
$$

First, for $L=L_{1}^{-1}$, it is immediate that $\mathrm{H}^{0}\left(X, L_{1}^{-1}\right)=0$ since $L_{1}$ is effective and nonzero, and because $\mathrm{h}^{0}\left(X, L_{1}\right)=2$ and $L_{1} \cdot L_{1}=0$, we conclude that $\mathrm{h}^{1}(X$, $\left.L_{1}^{-1}\right)=0$. It also follows that the complete linear system described by any of the $L_{i}$ is a genus one fibration on $X$.

Next, for $L=L_{1} \otimes L_{2}^{-1}$, we have $L^{2}=\left(L_{1}-L_{2}\right)^{2}=-4$, so $\mathrm{h}^{0}(L)-\mathrm{h}^{1}(L)+$ $\mathrm{h}^{2}(L)=0$. However, $L$ and $-L$ are not effective by genericity (since $L \cdot L_{1}=-2$ 
and $\left.(-L) \cdot L_{2}=-2\right)$, so $\mathrm{h}^{0}$ and $\mathrm{h}^{2}$ vanish (the latter by Serre duality). Therefore, $\mathrm{h}^{1}(L)=0$.

Similarly, $L=L_{1} \otimes L_{2} \otimes L_{3}^{-1}$ has $L^{2}=-4$, and furthermore $(-L) \cdot L_{3}=-4$, while $L \cdot L_{1}=L \cdot L_{2}=0$. We can conclude again by genericity that $L$ and $-L$ are ineffective. So $\mathrm{h}^{1}$ vanishes for this line bundle as well.

Finally, for $L=L_{1} \otimes L_{2} \otimes L_{3} \otimes L_{4}^{-1}$, we have $L^{2}=0$, so in fact $L$ or $-L$ must be effective. In fact, from equation (8), we see that $L=L_{5}^{(123)}+\sum E_{i}$, and it immediately follows that $-L$ is not effective (alternatively, the latter also follows from $\left.(-L) \cdot L_{i}<0\right)$. From the Gram matrices for the Picard groups in each case, one can see that $L \cdot E_{i}=-1$, which means that $E_{i}$ lie in the base locus of the linear system described by $L$. Therefore, $\mathrm{h}^{0}(L)=\mathrm{h}^{0}\left(L_{5}^{(123)}\right)=2$. We obtain $\mathrm{h}^{1}(L)=0$ from equation (10) above.

REMARK 7.7. We will treat several subvarieties of this moduli space (or rather, finite covers of them) through the various symmetrizations of the penteract, in subsequent sections. The comments in Remark 4.8 can be adapted to show that the genericity assumption of the lemma does not exclude these subvarieties.

The proof of surjectivity of the map (9) follows from three applications of the basepoint-free pencil trick. Therefore, the kernel of $\mu$ in (9) has dimension 2, and we obtain a penteract as an element of $\mathrm{H}^{0}\left(X, L_{1}\right) \otimes \mathrm{H}^{0}\left(X, L_{2}\right) \otimes \mathrm{H}^{0}(X$, $\left.L_{3}\right) \otimes \mathrm{H}^{0}\left(X, L_{4}\right) \otimes(\operatorname{ker} \mu)^{\vee}$, up to the action of $G$.

Proof of Theorem 7.1. It only remains to show that the two constructions described above are inverse to one another. Given a nonsingular K3 surface $X$ with appropriate line bundles $L_{1}, L_{2}, L_{3}$, and $L_{4}$ as in the statement of the theorem, let $Y_{1234}$ be the natural image of $X$ in $\mathbb{P}\left(\mathrm{H}^{0}\left(X, L_{1}\right)^{\vee}\right) \times \mathbb{P}\left(\mathrm{H}^{0}(X\right.$, $\left.\left.L_{2}\right)^{\vee}\right) \times \mathbb{P}\left(\mathrm{H}^{0}\left(X, L_{3}\right)^{\vee}\right) \times \mathbb{P}\left(\mathrm{H}^{0}\left(X, L_{4}\right)^{\vee}\right)$, and let $Y_{i j k}$ for $\{i, j, k\} \subset\{1,2,3,4\}$ be the projection onto the $i$ th, $j$ th, and $k$ th factors.

On the other hand, construct the penteract $A \in \mathrm{H}^{0}\left(X, L_{1}\right) \otimes \mathrm{H}^{0}\left(X, L_{2}\right) \otimes \mathrm{H}^{0}(X$, $\left.L_{3}\right) \otimes \mathrm{H}^{0}\left(X, L_{4}\right) \otimes(\operatorname{ker} \mu)^{\vee}$ from $\left(X, L_{1}, L_{2}, L_{3}, L_{4}\right)$ as above, and let $X_{1234}$ and $X_{i j k}$ be the K3 surfaces constructed from $A$ in the usual way. By the construction of $A$ as the kernel of $\mu$, we have $A(v, w, x, y, \cdot)=0$ for any point $(v, w, x$, $y) \in Y_{1234}$, so $Y_{1234} \subset X_{1234}$ and $Y_{i j k} \subset X_{i j k}$. We claim that $X_{1234}=Y_{1234}$ and $X_{i j k}=Y_{i j k}$ as sets and as varieties.

At least one of the tridegree $(2,2,2)$ polynomials $f_{i j k}$ defining $X_{i j k}$ is not identically zero; for such a variety $X_{i j k}$, we have that $X_{i j k}$ and $Y_{i j k}$ are both given by nonzero tridegree $(2,2,2)$ forms and thus must be the same variety, and similarly for $X_{1234}$ and $Y_{1234}$. Moreover, because $Y_{1234}$ is assumed to be nonsingular, the tensor $A$ is nondegenerate. 
Conversely, given a nondegenerate penteract $A \in V_{1} \otimes V_{2} \otimes V_{3} \otimes V_{4} \otimes V_{5}$, let $X$ be the nonsingular K3 surface $X_{1234}$ constructed from $A$ and $L_{1}, L_{2}, L_{3}$, $L_{4}$ be the line bundles on $X$. Then the vector spaces $V_{i}$ and $\mathrm{H}^{0}\left(X, L_{i}\right)$ are naturally isomorphic for $1 \leqslant i \leqslant 4$, and $V_{5}^{\vee}$ can be identified with the kernel of the multiplication map $\mu$ from above. With these identifications, the penteract constructed from this geometric data is well defined and $G$-equivalent to the original $A$.

7.4. Automorphisms. Given a nondegenerate penteract $A$, we may consider the following composition of the isomorphisms from Section 7.1:

$$
\alpha_{34,5}: X_{1234} \rightarrow X_{124} \rightarrow X_{1245} \rightarrow X_{125} \rightarrow X_{1235} \rightarrow X_{123} \rightarrow X_{1234}
$$

Since each map is an isomorphism, the entire composition is an automorphism of $X_{1234}$. It is easy to see that it is not the identity, however; in fact, a point $\left(v_{0}, w_{0}\right.$, $\left.x_{0}, y_{0}\right) \in X_{1234} \subset \mathbb{P}\left(V_{1}^{\vee}\right) \times \mathbb{P}\left(V_{2}^{\vee}\right) \times \mathbb{P}\left(V_{3}^{\vee}\right) \times \mathbb{P}\left(V_{4}^{\vee}\right)$ is sent to $\left(v_{0}, w_{0}, x_{1}, y_{1}\right)$, where $x_{0}$ and $x_{1}$ are the two solutions for $x$ in the equation $\operatorname{det} A\left(v_{0}, w_{0}, x, \cdot\right.$, $\cdot)=0\left(\right.$ and similarly for $y_{0}$ and $\left.y_{1}\right)$.

We may similarly define automorphisms $\alpha_{k l, m}$ of $X_{i j k l}$ for any permutation $\{i$, $j, k, l, m\}$ of $\{1,2,3,4,5\}$ (where the ordering of the indices in the subscript of $\alpha$, but not of $X$, is relevant). For example, the automorphisms $\alpha_{k l, m}$ and $\alpha_{l k, m}$ of $X_{i j k l}$ are inverse to one another (and actually the same, as described below), but $\alpha_{k m, l}$ is an automorphism of $X_{i j k m}$.

A more geometric way to describe these automorphisms is by viewing $X_{i j k l}$ as a double cover of $\mathbb{P}\left(V_{i}^{\vee}\right) \times \mathbb{P}\left(V_{j}^{\vee}\right)$; then $\alpha_{k l, m}$ switches the two sheets of this double cover. It is clear that all of these automorphisms have order two, and thus $\alpha_{k l, m}=\alpha_{l k, m}$.

Using the relation (7) and its analogues, we may easily compute how $\alpha_{k l, m}$ acts on the Néron-Severi lattice. For example, the automorphism $\alpha_{34,5}$ is equivalent to the action of the matrix

$$
\left(\begin{array}{cccc}
1 & 0 & 0 & 0 \\
0 & 1 & 0 & 0 \\
2 & 2 & -1 & 0 \\
2 & 2 & 0 & -1
\end{array}\right)
$$

on $\mathrm{NS}(X)$. Conjugating $(11)$ by $4 \times 4$ permutation matrices yields all six automorphisms of the form $\alpha_{k l, 5}$ for $k, l \in\{1,2,3,4\}$.

For very general $X$, the group $\Gamma_{\text {pent }}$ generated by these automorphisms $\alpha_{k l, m}$ turns out to have index 60 in the orthogonal group $O(\mathrm{NS}(X), \mathbb{Z})$ of $\mathrm{NS}(X)$, and therefore also finite index in $\operatorname{Aut}(X)$. (We are grateful to Igor Rivin for performing this interesting computation.) One way to visualize these automorphisms is by 

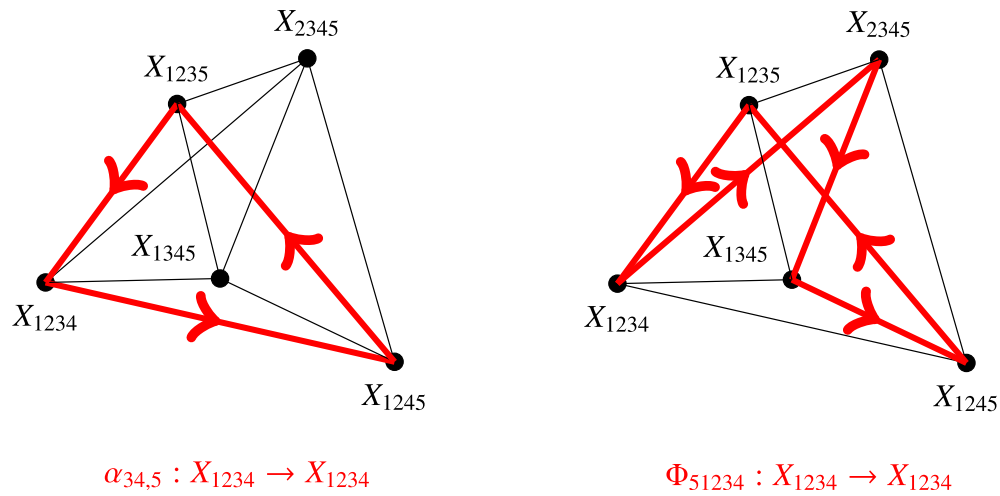

Figure 2. Some automorphisms of the K3 surface associated to a penteract.

placing each of the five $X_{i j k l}$ on a vertex of the 5-cell (a.k.a. 4-simplex) and by viewing each edge as the isomorphism from $X_{i j k l}$ to $X_{i j k m}$ through $X_{i j k}$ (again, as defined in Section 7.1); see Figure 2. Then each $\alpha_{k l, m}$ is the traversal of a triangle in the 1-dimensional boundary of the 5-cell.

These $\alpha_{k l, m}$ 's may be composed to yield nontrivial automorphisms that are 4and 5-cycles on the boundary of the 5-cell. The 4-cycles give automorphisms that preserve one of the genus one fibrations and act by translation by a section of the Jacobian fibration (see [16, Section 6.2]). It is easy to check that they have fixed points on reducible fibers of the fibrations.

An example of a 5-cycle is the automorphism

$$
\Phi_{51234}: X_{1234} \rightarrow X_{2345} \rightarrow X_{1345} \rightarrow X_{1245} \rightarrow X_{1235} \rightarrow X_{1234}
$$

which is the composition $\alpha_{34,5} \circ \alpha_{23,5} \circ \alpha_{12,5}$. Applying $\Phi_{51234}$ to $X$ induces the action of the matrix

$$
\left(\begin{array}{cccc}
-1 & 0 & 2 & 2 \\
-2 & 1 & 2 & 4 \\
-4 & 2 & 5 & 6 \\
-6 & 2 & 8 & 11
\end{array}\right)
$$

on $\Lambda$ in $\mathrm{NS}(X)$. By symmetry, all of the 5-cycles that meet all 5 vertices act in a similar way on $\mathrm{NS}(X)$. As we will see in Section 17.4, these 5-cycle automorphisms of $X$ turn out to be fixed-point-free in general and have positive entropy.

As mentioned in the introduction, the elements of $\Gamma_{\text {pent }}$ are often fixed-pointfree and of positive entropy. One obtains many different quadratic and quartic Salem polynomials as the characteristic polynomials of these automorphisms. In 
particular, the Néron-Severi lattice $\mathrm{NS}(X)$ is isomorphic to $U(2) \oplus A_{2}$ (2) for a very general $X$ in our family. Therefore, $\operatorname{Aut}(X)$ has finite index in $O\left(U \oplus A_{2}\right)$, which is commensurable to $\mathrm{SL}_{2}(\mathbb{Z}[\omega]$ ) where $\omega$ denotes a third root of unity. (We are grateful to Curt McMullen for pointing out this commensurability.) One way to see this commensurability is as follows: consider the Hermitian form over $\mathbb{Z}[\omega]$ with matrix given by

$$
M=\left(\begin{array}{cc}
x & z-\omega w \\
z-\omega^{2} w & y
\end{array}\right) .
$$

The discriminant of this form (that is, the determinant of the matrix) is $x y-$ $\left(z^{2}+z w+w^{2}\right)$, which is half the quadratic form corresponding to the lattice $U \oplus A_{2}$. Therefore, it is enough to show that a finite-index subgroup of the group $\mathrm{SL}_{2}(\mathbb{Z}[\omega])$ acts as a group of automorphisms of the Hermitian form (13). This is readily accomplished by considering the action $g \cdot M=g M g^{\dagger}$, where $g \in \mathrm{SL}_{2}(\mathbb{Z}[\omega])$ and $g^{\dagger}$ is the conjugate transpose of $g$. We omit checking the standard details, referring the interested reader to, for example, [22, Section 13.9, page 317$]$. (We may also see this commensurability geometrically by comparing the automorphism group of the abelian surface $E \times E$ (where $E=\mathbb{C} / \mathbb{Z}[\omega]$ is the elliptic curve of $j$-invariant 0 ) with the automorphisms of its transcendental lattice $U(-1) \oplus A_{2}(-1)$. See also Aurel Page's answer in [64], which outlines a proof that $O\left(U \oplus A_{2}\right)$ is in fact isomorphic to $\mathrm{PGL}_{2}(\mathbb{Z}[\omega])$.)

The Salem polynomial corresponding to the action of $g \in \mathrm{SL}_{2}(\mathbb{Z}[\omega])$ on the Hermitian form $M$ is $p_{g}(T)=T^{4}-e e^{\prime} T^{3}+\left(e^{2}+e^{\prime 2}-2\right) T^{2}-e e^{\prime} T+1$, where $e=$ Trace $(g)$ and $e^{\prime}$ is the conjugate of $e$. The splitting field of this quartic polynomial has Galois group isomorphic to the dihedral group $D_{4}$ of order 8 , and it is also the splitting field of

$$
\begin{aligned}
q_{g}(T) & =\operatorname{Norm}_{\mathbb{Q}(\omega)[T] / \mathbb{Q}[T]} \operatorname{det}(g-T \cdot \mathrm{Id}) \\
& =\operatorname{Norm}_{\mathbb{Q}(\omega)[T] / \mathbb{Q}[T]}\left(T^{2}-e T+1\right) \\
& =T^{4}-\left(e+e^{\prime}\right) T^{3}+\left(2+e e^{\prime}\right) T^{2}-\left(e+e^{\prime}\right) T+1
\end{aligned}
$$

over $\mathbb{Q}$. In fact, the fields $K_{p}$ and $K_{q}$ obtained by adjoining a root of $p_{g}(T)$ and $q_{g}(T)$, respectively, to $\mathbb{Q}$ are dual $D_{4}$-quartic fields, that is, they are the fixed fields of the two subgroups of order 2 (up to conjugacy) in $D_{4}$ which are interchanged by the outer involution of $D_{4}$. In particular, the quadratic resolvent field of $K_{p}$ is $\mathbb{Q}(\omega)$.

It is then easy to deduce that the quadratic Salem polynomials of the automorphism group of $X$ generate all real quadratic fields, while the quartic Salem polynomials generate all $D_{4}$-quartic fields whose quadratic resolvent field is $\mathbb{Q}(\omega)$. Below, we indicate how to explicitly find automorphisms to prove the stronger statement in the introduction about specific quadratic Salem polynomials 
of the form $x^{2}-\left(4 n^{2} \pm 2\right) x+1$ and $x^{2}-\left(12 n^{2} \pm 2\right) x+1$. A similar analysis using the unit group of the quartic field $\mathbb{Q}(\omega)[T] /\left(T^{2}-e T+1\right)$ gives the second half of our assertion.

Let

$$
\gamma_{1}=\alpha_{34,5} \circ \alpha_{24,5}=\left(\begin{array}{cccc}
1 & 0 & 0 & 0 \\
2 & -1 & 2 & 0 \\
6 & -2 & 3 & 0 \\
4 & -2 & 2 & 1
\end{array}\right) \quad \text { and } \quad \gamma_{2}=\alpha_{13,5} \circ \alpha_{12,5}=\left(\begin{array}{cccc}
1 & -2 & 2 & 4 \\
0 & -1 & 2 & 2 \\
0 & -2 & 3 & 6 \\
0 & 0 & 0 & 1
\end{array}\right) \text {. }
$$

Then it is easily verified that for $k \in \mathbb{Z}$, the automorphism $\gamma_{1}^{k} \gamma_{2}$ produces the Salem polynomial

$$
x^{2}-\left(4(2 k+1)^{2}-2\right) x+1 .
$$

A positive proportion of the polynomials $x^{2}-\left(4 n^{2}+2\right) x+1$ and $x^{2}-\left(12 n^{2} \pm\right.$ 2) $x+1$ may also be obtained as Salem polynomials of penteract automorphisms in a similar way:

(a) for $\gamma_{1}=\alpha_{34,5} \circ \alpha_{24,5}$ and $\gamma_{2}=\alpha_{14,5}$, the Salem polynomial corresponding to the automorphism $\gamma_{1}^{k} \gamma_{2}$ is $x^{2}-\left(4(2 k)^{2}+2\right) x+1$;

(b) for $\gamma_{1}=\alpha_{34,5} \circ \alpha_{24,5} \circ \alpha_{34,5}$ and $\gamma_{2}=\alpha_{23,5} \circ \alpha_{12,5}$, the Salem polynomial corresponding to the automorphism $\gamma_{1} \gamma_{2}^{k}$ is $x^{2}-\left(12(2 k)^{2}-2\right) x+1$;

(c) for $\gamma_{1}=\alpha_{12,5} \circ \alpha_{34,5}$ and $\gamma_{2}=\alpha_{23,5} \circ \alpha_{34,5} \circ \alpha_{24,5} \circ \alpha_{34,5}$, the Salem polynomial corresponding to the automorphism $\gamma_{1} \gamma_{2}^{k}$ is $x^{2}-\left(12(2 k+1)^{2}+2\right) x+1$.

Obtaining the Salem polynomials of the form (14) is sufficient to deduce that all real quadratic fields occur as the splitting fields of Salem polynomials of automorphisms of general K3 surfaces in our penteract family. To see this, for each discriminant $D$ of a real quadratic field, we wish to show the existence of a pair $(m, n)$ of positive integers with $m=2 k+1$ such that $D n^{2}=\left(4 m^{2}-2\right)^{2}-4=$ $16 m^{2}\left(m^{2}-1\right)$, or equivalently, the existence of a pair $\left(m, n^{\prime}\right)$ of positive integers with $m$ odd such that $m^{2}-D n^{\prime 2}=1$ (for we may then set $n$ to be $4 m n^{\prime}$ ). The latter Brahmagupta-Pell equation is well known to have infinitely many positive integer solutions $\left(m, n^{\prime}\right)$ for every discriminant $D$, even with the restriction that $m$ is odd, proving the claim.

\section{Doubly symmetric penteracts: $2 \otimes 2 \otimes 2 \otimes \operatorname{Sym}^{2}(2)$}

We now consider doubly symmetric penteracts, namely elements of $V=V_{1} \otimes$ $V_{2} \otimes V_{3} \otimes \operatorname{Sym}^{2} V_{4}$ for 2-dimensional $F$-vector spaces $V_{1}, V_{2}, V_{3}$, and $V_{4}$, with an action of the group $\operatorname{GL}\left(V_{1}\right) \times \operatorname{GL}\left(V_{2}\right) \times \operatorname{GL}\left(V_{3}\right) \times \operatorname{GL}\left(V_{4}\right)$. Since the space of 
doubly symmetric penteracts maps naturally into the space of all penteracts from Section 7 after the identification of $V_{4}$ and $V_{5}$, one may understand the orbits of doubly symmetric penteracts by using constructions from Theorem 7.1. We find that these orbits correspond to certain K3 surfaces of Picard rank at least 9 over $\bar{F}$ :

THEOREM 8.1. Let $V=V_{1} \otimes V_{2} \otimes V_{3} \otimes \mathrm{Sym}^{2} V_{4}$ for 2-dimensional F-vector spaces $V_{1}, V_{2}, V_{3}$, and $V_{4}$. Let $G^{\prime}=\mathrm{GL}\left(V_{1}\right) \times \mathrm{GL}\left(V_{2}\right) \times \mathrm{GL}\left(V_{3}\right) \times \operatorname{GL}\left(V_{4}\right)$ and let $G$ be the quotient of $G^{\prime}$ by the kernel of the natural multiplication map $\mathbb{G}_{m} \times \mathbb{G}_{m} \times \mathbb{G}_{m} \times \mathbb{G}_{m} \rightarrow \mathbb{G}_{m}$ sending $\left(\gamma_{1}, \gamma_{2}, \gamma_{3}, \gamma_{4}\right) \mapsto \gamma_{1} \gamma_{2} \gamma_{3} \gamma_{4}^{2}$. Let $\Lambda$ be the lattice whose Gram matrix is

$$
\left(\begin{array}{ccccccccc}
0 & 2 & 2 & 2 & 0 & 0 & 0 & 0 & 0 \\
2 & 0 & 2 & 2 & 0 & 0 & 0 & 0 & 0 \\
2 & 2 & 0 & 2 & 0 & 0 & 0 & 0 & 0 \\
2 & 2 & 2 & 0 & 1 & 1 & 1 & 1 & 1 \\
0 & 0 & 0 & 1 & -2 & 0 & 0 & 0 & 0 \\
0 & 0 & 0 & 1 & 0 & -2 & 0 & 0 & 0 \\
0 & 0 & 0 & 1 & 0 & 0 & -2 & 0 & 0 \\
0 & 0 & 0 & 1 & 0 & 0 & 0 & -2 & 0 \\
0 & 0 & 0 & 1 & 0 & 0 & 0 & 0 & -2
\end{array}\right),
$$

and let $S=\left\{e_{1}, e_{2}, e_{3}, e_{4}\right\}$. Then the $G(F)$-orbits of an open subset of $V(F)$ are in bijection with the $F$-points of an open subvariety of the moduli space $\mathcal{M}_{\Lambda, S}$ of $K 3$ surfaces $X$ lattice-polarized by $(\Lambda, S)$.

Many of the constructions in Section 7 apply, but there are some important differences. For a general penteract, the ten different $\mathrm{K} 3$ surfaces in $\mathbb{P}^{1} \times \mathbb{P}^{1} \times \mathbb{P}^{1}$ are nonsingular. For a doubly symmetric penteract $A$, however, the locus of $(r, s$, $t$ ) where $A(r, s, t, \cdot, \cdot)$ is identically zero is 0 -dimensional and of degree 6 , as it is given as the intersection of three $(1,1,1)$ forms on $\mathbb{P}\left(V_{1}^{\vee}\right) \times \mathbb{P}\left(V_{2}^{\vee}\right) \times \mathbb{P}\left(V_{3}^{\vee}\right)$. Therefore, the $\mathrm{K} 3$ surface $X_{123}$ defined by det $A(r, s, t, \cdot, \cdot)=0$ will have, in general, 6 isolated (rank) singularities over $\bar{F}$. These singularities of $X_{123}$ are blown up by the map $X_{1234} \rightarrow X_{123}$, and while these singularities may not be individually defined over $F$, the entire degree 6 subscheme is defined over $F$. It is easy to check that all of the other $X_{i j k}$ coming from $A$ are generically nonsingular.

8.1. Néron-Severi lattice. To compute the Néron-Severi group of the nonsingular K3 surface $X_{1234}$ here, we observe that there still are line bundles $L_{i}$ on $X_{1234}$ coming from pulling back $\mathcal{O}_{\mathbb{P}\left(V_{i}^{\vee}\right)}(1)$ to $X_{1234}$ for $1 \leqslant i \leqslant 4$. In addition, over $\bar{F}$, there are six exceptional fibers $E_{i}$ for $1 \leqslant i \leqslant 6$, coming from the blow-ups of the 6 singularities in $X_{123}$; the sum of these $E_{i}$ is a divisor defined 
over $F$. It is easy to compute all of the intersection numbers: the only nonzero ones are $L_{i} \cdot L_{j}=2\left(1-\delta_{i j}\right), L_{4} \cdot E_{i}=1$ for $1 \leqslant i \leqslant 6$, and $E_{i}^{2}=-2$.

Recall from Lemma 7.2 that there is a relation among the line bundles; here, it is slightly simplified because of the symmetry (that is, $L_{4}$ and $L_{5}^{123}$ are isomorphic):

$$
L_{1}+L_{2}+L_{3}=2 L_{4}+\sum_{i=1}^{6} E_{i} .
$$

We thus expect the Néron-Severi group to generically have rank 9, and in fact, the intersection matrix of all of these divisor classes may be reduced to the matrix (15), which is the intersection matrix for $L_{1}, \ldots, L_{4}, E_{1}, \ldots, E_{5}$. Note that the lattice they span has discriminant $2^{8}$.

PROPOSITION 8.2. For a very general $X$ in this family of $K 3$ surfaces, $\overline{\mathrm{NS}}(X)$ is spanned over $\mathbb{Z}$ by $L_{1}, L_{2}, L_{4}$, and the $E_{i}, i=1, \ldots, 6$.

Proof. Since the moduli space here has dimension $8 \cdot 3-13=11$, the Picard number of a very general $X$ is at most 9 . It is enough to check that the lattice $L$ spanned by these divisors is 2-saturated. Computation of the discriminant group shows that any element of the dual lattice may be written as

$$
D=\frac{1}{2}\left(c_{1} L_{1}+c_{2} L_{2}+c_{4} L_{4}\right)+\frac{1}{4} \sum d_{i} E_{i}
$$

with $c_{i}, d_{i}$ integers. First, by Lemma 2.3 , no divisor of the form $\left(\sum e_{i} E_{i}\right) / 2$, with $e_{i}$ integers, can be in $\overline{\mathrm{NS}}(X)$, unless all the $e_{i}$ are even. It follows that in the expression for $D$, all the $d_{i}$ must be even, as $2 D-c_{1} L_{1}-c_{2} L_{2}-c_{4} L_{4}$ would otherwise be a counterexample to the above observation. So we may assume that $D$ has the form

$$
D=\frac{1}{2}\left(c_{1} L_{1}+c_{2} L_{2}+c_{4} L_{4}+\sum_{i=1}^{6} e_{i} E_{i}\right) .
$$

Intersection with $E_{i}$ shows that $c_{4}$ is an even integer, so we may assume it is zero. If $c_{1}$ and $c_{2}$ are even, then we get a contradiction to Lemma 2.3 as above. At least one of $c_{1}$ and $c_{2}$ is odd, and if both are not odd, we may construct a divisor $D^{\prime} \in \overline{\mathrm{NS}}(X)$ by reversing the roles of $L_{1}$ and $L_{2}$, by symmetry. Then $D+D^{\prime}$ has the same shape as (17), with both coefficients $c_{1}$ and $c_{2}$ odd. So we may assume $c_{1}=c_{2}=1$ and all $e_{i} \in\{0,1\}$ by subtracting an element of $L$.

The self-intersection of $D$ is $1-\sum e_{i}^{2} / 2$ and is then even, so $\sum e_{i}^{2}=2$ or 6. If exactly two of the $e_{i}$ are 1 , say $E_{1}$ and $E_{2}$, then by symmetry each of the divisors $\left(L_{1}+L_{2}+E_{i}+E_{j}\right) / 2$ is in $\overline{\mathrm{NS}}(X)$. Subtracting two of these, we see that 
$\left(E_{1}+E_{2}+E_{3}+E_{4}\right) / 2$ is in $\overline{\mathrm{NS}}(X)$, which contradicts Lemma 2.3. Finally, if all six $e_{i}$ are 1, then another application of symmetry shows that $D^{\prime}=\left(L_{1}+L_{3}+\sum E_{i}\right) \in$ $\overline{\mathrm{NS}}(X)$. Therefore, $D-D^{\prime}=\left(L_{1}-L_{3}\right) / 2 \in \overline{\mathrm{NS}}(X)$, which is impossible since it has odd self-intersection.

Corollary 8.3. The divisors $L_{1}, \ldots, L_{4}, E_{1}, \ldots, E_{5}$ form a basis for $\overline{\mathrm{NS}}(X)$, for $X$ very general.

\subsection{Moduli problem. We now complete the proof of Theorem 8.1.}

Proof of Theorem 8.1. The constructions in both directions almost exactly follow those for Theorem 7.1. Given a doubly symmetric penteract, we obtain the K3 surfaces $X_{i j k}$ and $X_{i j k l}$ with the divisor classes (and intersection matrix) as described above.

On the other hand, given such a K3 surface $X$ lattice-polarized by $(\Lambda, S)$, we must show that the penteract $A$ constructed by the reverse map of Section 7.3 is doubly symmetric under an identification of two of the vector spaces. Let $L_{1}, L_{2}$, $L_{3}$, and $L_{4}$ be line bundles corresponding to the four elements of $S$ (in order). These are the line bundles used to construct the penteract $A \in \mathrm{H}^{0}\left(X, L_{1}\right) \otimes \mathrm{H}^{0}(X$, $\left.L_{2}\right) \otimes \mathrm{H}^{0}\left(X, L_{3}\right) \otimes \mathrm{H}^{0}\left(X, L_{4}\right) \otimes(\operatorname{ker} \mu)^{\vee}$, where $\mu$ is the multiplication map (9).

Note that $A$ in turn gives rise to isomorphic K3 surfaces and line bundles, as well as a fifth line bundle, say $L_{5}^{(123)}$, via the map from $X_{1234} \rightarrow X_{123}--\rightarrow X_{1235} \rightarrow$ $\mathbb{P}(\operatorname{ker} \mu)$. In addition, from the intersection matrix (15), we see that there are six singularities on $X_{123}$ which are blown up in $X_{1234}$ (whose exceptional fibers correspond to the last six rows/columns of the intersection matrix). Thus, using the relation (8) and the intersection matrix (15), we find that $L_{4}$ and $L_{5}^{(123)}$ are in fact isomorphic. Therefore, we may identify the vector spaces $V_{4}:=\mathrm{H}^{0}\left(X, L_{4}\right)$ and $V_{5}:=(\operatorname{ker} \mu)^{\vee}$.

With this identification, the maps from $X_{123}$ to $\mathbb{P}\left(V_{4}^{\vee}\right)$ and $\mathbb{P}\left(V_{5}^{\vee}\right)$ are identical and given in the usual way by taking the appropriate kernels of $A(v, w, x, \cdot$, $\cdot) \in V_{4} \otimes V_{5}$ for $(v, w, x) \in X_{123}$. The remaining key idea is very simple to check (for example, explicitly) in this case: for a rank-one element $\zeta \in V \otimes V$ for a 2-dimensional $F$-vector space $V$, if $\zeta(v, \cdot)=0$ and $\zeta(\cdot, v)=0$, then $\zeta$ is in fact in the symmetric subspace $\operatorname{Sym}^{2} V$ of $V \otimes V$. Since $X_{123}$ spans the ambient space $\mathbb{P}\left(\mathrm{H}^{0}\left(X, L_{1}\right)^{\vee}\right) \times \mathbb{P}\left(\mathrm{H}^{0}\left(X, L_{2}\right)^{\vee}\right) \times \mathbb{P}\left(\mathrm{H}^{0}\left(X, L_{3}\right)^{\vee}\right)$, the penteract $A$ is in fact symmetric, that is, an element of $\mathrm{H}^{0}\left(X, L_{1}\right) \otimes \mathrm{H}^{0}\left(X, L_{2}\right) \otimes \mathrm{H}^{0}(X$, $\left.L_{3}\right) \otimes \operatorname{Sym}^{2} \mathrm{H}^{0}\left(X, L_{4}\right)$, as desired.

8.3. Automorphisms. The automorphisms $\alpha_{i j, k}$ defined in Section 7.4 are again automorphisms of the K3 surfaces obtained from doubly symmetric 
penteracts. Of course, because of the symmetry in this case, some of these are the same automorphism, for example, $\alpha_{14,2}=\alpha_{15,2}$.

Moreover, the action of the 5-cycles on the Néron-Severi lattice is different, since we now have to take the exceptional divisor classes into account. To compute this action for the generic K3 surface in this family, we employ the same methods as for the doubly symmetric Rubik's revenge, namely, repeated applications of the relation (8) and computations of intersection numbers. For example, the action of

$$
\Phi_{54321}: X_{1234} \rightarrow X_{1235} \rightarrow X_{1245} \rightarrow X_{1345} \rightarrow X_{2345} \rightarrow X_{1234}
$$

on $\overline{\mathrm{NS}}(X)$ here is given by the matrix

$$
\left(\begin{array}{lllllllll}
5 & 2 & -4 & 6 & 0 & 0 & 0 & 0 & 0 \\
2 & 1 & -2 & 4 & 0 & 0 & 0 & 0 & 0 \\
2 & 0 & -1 & 2 & 0 & 0 & 0 & 0 & 0 \\
1 & 1 & -1 & 1 & 0 & 0 & 0 & 0 & 0 \\
1 & 0 & -1 & 2 & 1 & 0 & 0 & 0 & 0 \\
1 & 0 & -1 & 2 & 0 & 1 & 0 & 0 & 0 \\
1 & 0 & -1 & 2 & 0 & 0 & 1 & 0 & 0 \\
1 & 0 & -1 & 2 & 0 & 0 & 0 & 1 & 0 \\
1 & 0 & -1 & 2 & 0 & 0 & 0 & 0 & 1
\end{array}\right) .
$$

We will look at this automorphism again in Section 17.4.

By symmetry, all of the 5-cycles that meet all five models $X_{i j k l}$ are either analogous to $\Phi_{54321}$ above or to $\Phi_{53421}$. In the latter case, the induced action on the line bundles $L_{i}$ is similar to that of $\Phi$ in the penteract case (from which the action on the divisors $E_{j}$ may be immediately deduced). These two types of automorphisms will be shown in Section 17.4 to be fixed-point-free in general and of positive entropy.

\section{Triply symmetric penteracts: $2 \otimes 2 \otimes \operatorname{Sym}^{3}(2)$}

Suppose we now have a penteract that is symmetric in the last three coordinates. We prove that the general orbits of such tensors correspond to certain K3 surfaces with Picard rank at least 14 over $\bar{F}$ :

THEOREM 9.1. Let $V=V_{1} \otimes V_{2} \otimes \mathrm{Sym}^{3} V_{3}$ for 2-dimensional $F$-vector spaces $V_{1}, V_{2}, V_{3}$. Let $G^{\prime}=\operatorname{GL}\left(V_{1}\right) \times \operatorname{GL}\left(V_{2}\right) \times \operatorname{GL}\left(V_{3}\right)$ act on $V$, and let $G$ be the quotient of $G^{\prime}$ by the kernel of the map $\mathbb{G}_{m} \times \mathbb{G}_{m} \times \mathbb{G}_{m} \rightarrow \mathbb{G}_{m}$ sending $\left(\gamma_{1}, \gamma_{2}\right.$, $\left.\gamma_{3}\right) \mapsto \gamma_{1} \gamma_{2} \gamma_{3}^{3}$. Let $\Lambda$ be the lattice whose Gram matrix is 


$$
\left(\begin{array}{cccccccccccccc}
0 & 2 & 2 & 2 & 0 & 0 & 0 & 0 & 0 & 0 & 0 & 0 & 0 & 0 \\
2 & 0 & 2 & 2 & 0 & 0 & 0 & 0 & 0 & 0 & 0 & 0 & 0 & 0 \\
2 & 2 & 0 & 2 & 0 & 1 & 0 & 1 & 0 & 1 & 0 & 1 & 0 & 1 \\
2 & 2 & 2 & 0 & 1 & 0 & 1 & 0 & 1 & 0 & 1 & 0 & 1 & 0 \\
0 & 0 & 0 & 1 & -2 & 1 & 0 & 0 & 0 & 0 & 0 & 0 & 0 & 0 \\
0 & 0 & 1 & 0 & 1 & -2 & 0 & 0 & 0 & 0 & 0 & 0 & 0 & 0 \\
0 & 0 & 0 & 1 & 0 & 0 & -2 & 1 & 0 & 0 & 0 & 0 & 0 & 0 \\
0 & 0 & 1 & 0 & 0 & 0 & 1 & -2 & 0 & 0 & 0 & 0 & 0 & 0 \\
0 & 0 & 0 & 1 & 0 & 0 & 0 & 0 & -2 & 1 & 0 & 0 & 0 & 0 \\
0 & 0 & 1 & 0 & 0 & 0 & 0 & 0 & 1 & -2 & 0 & 0 & 0 & 0 \\
0 & 0 & 0 & 1 & 0 & 0 & 0 & 0 & 0 & 0 & -2 & 1 & 0 & 0 \\
0 & 0 & 1 & 0 & 0 & 0 & 0 & 0 & 0 & 0 & 1 & -2 & 0 & 0 \\
0 & 0 & 0 & 1 & 0 & 0 & 0 & 0 & 0 & 0 & 0 & 0 & -2 & 1 \\
0 & 0 & 1 & 0 & 0 & 0 & 0 & 0 & 0 & 0 & 0 & 0 & 1 & -2
\end{array}\right),
$$

and let $S=\left\{e_{1}, e_{2}, e_{3}, e_{4}\right\}$. Then the $G(F)$-orbits of an open subset of $V(F)$ are in bijection with the $F$-points of an open subvariety of the moduli space $\mathcal{M}_{\Lambda, S}$ of $K 3$ surfaces $X$ lattice-polarized by $(\Lambda, S)$.

9.1. Néron-Severi lattice. Note that a triply symmetric penteract is also doubly symmetric in any two of the last three coordinates. This implies, from Section 8, that the K3 surface $X_{123}\left(=X_{124}=X_{125}\right)$ has at least six rank singularities (over $\bar{F}$ ), and a numerical example shows us that generically there are no other singularities. Meanwhile, the other surfaces $X_{134}$ and $X_{234}$ are generically nonsingular, as are all the $X_{i j k l}$.

The maps of the type $X_{1234} \rightarrow X_{123}$ blow up the six singular points on $X_{123}$, and thus $X_{1234}$ contains six lines; call the associated divisor classes $P_{i}$ for $1 \leqslant$ $i \leqslant 6$. There is also a map $X_{1234} \rightarrow X_{124}$, defined by the identical equations after switching the 3rd and 4th coordinates, so there are at least twelve lines in $X_{1234}$; call the six lines coming from this map $Q_{i}$ for $1 \leqslant i \leqslant 6$. These twelve lines occur in pairs, say $\left(P_{i}, Q_{i}\right)$ for $1 \leqslant i \leqslant 6$, which are flipped by the birational involution $X_{123} \rightarrow X_{1234} \rightarrow X_{124}=X_{123}$.

Recall that there are line bundles $L_{1}, L_{2}, L_{3}$, and $L_{4}$ on $X_{1234}$ coming from the pullback of $\mathcal{O}_{\mathbb{P}\left(V_{i}^{\vee}\right)}(1)$, and by Lemma 7.2, we have the relations

$$
L_{1}+L_{2}+L_{3}=2 L_{4}+\sum_{i=1}^{6} P_{i} \quad \text { and } \quad L_{1}+L_{2}+L_{4}=2 L_{3}+\sum_{i=1}^{6} Q_{i} .
$$

Each of the six pairs of lines $\left(P_{i}, Q_{i}\right)$ determines a single point of intersection. Explicitly, if the associated singular point on $X_{123}$ is $(v, w, x) \in \mathbb{P}\left(V_{1}^{\vee}\right) \times \mathbb{P}\left(V_{2}^{\vee}\right) \times$ 
$\mathbb{P}\left(V_{3}^{\vee}\right)$, then the intersection point is $(v, w, x, x) \in \mathbb{P}\left(V_{1}^{\vee}\right) \times \mathbb{P}\left(V_{2}^{\vee}\right) \times \mathbb{P}\left(V_{3}^{\vee}\right) \times$ $\mathbb{P}\left(V_{3}^{\vee}\right)$. These are the only six intersection points among all of the $P_{i}$ and $Q_{j}$, as $\left(\sum P_{i}\right) \cdot\left(\sum Q_{j}\right)=6$.

Another way to see that each of these pairs of lines intersect once (and do not intersect any other lines) is to view $X_{123}$ as a double cover of $\mathbb{P}\left(V_{1}^{\vee}\right) \times \mathbb{P}\left(V_{2}^{\vee}\right)$, branched along a bidegree $(4,4)$ curve. One computes that there are exactly six $A_{2}$ singularities on that curve.

The map from $X_{1234}$ to $\mathbb{P}\left(V_{1}^{\vee}\right)$ is a genus one fibration whose discriminant as a binary form on $V_{1}$ has degree 24 , and it factors as the cube of a degree six form times an irreducible degree six form. Therefore, the genus one fibration has six reducible fibers of type $\mathrm{I}_{3}$ (in the sense of Kodaira [43, 44]). These reducible fibers each consist of three lines in a 'triangle'; a distinguished pair of these lines in each triangle together give us the six pairs of lines described previously.

As a consequence, the Néron-Severi lattice (over $\bar{F}$ ) has rank at least $2 \cdot 6+2=$ 14. It is straightforward to compute the intersection numbers of all of the known divisor classes (the four line bundles from pulling back $\mathcal{O}_{\mathbb{P}\left(V_{i}^{\vee}\right)}(1)$ for $1 \leqslant i \leqslant 4$ and the two distinguished lines in each of the six triangles). The only nonzero intersection numbers are

$$
\begin{aligned}
& L_{i} \cdot L_{j}=2 \quad \text { for } i \neq j, \quad L_{3} \cdot Q_{i}=1, \quad L_{4} \cdot P_{i}=1 \text {, } \\
& P_{i}^{2}=Q_{i}^{2}=-2, \quad P_{i} \cdot Q_{i}=1 .
\end{aligned}
$$

Taking the basis $\left\{L_{1}, \ldots, L_{4}, P_{1}, Q_{1}, \ldots, P_{5}, Q_{5}\right\}$, one obtains the lattice with Gram matrix (19). This lattice has discriminant -324 .

Proposition 9.2. For a very general $X$ in this family of $K 3$ surfaces, $\overline{\mathrm{NS}}(X)$ is spanned over $\mathbb{Z}$ by $L_{1}, L_{2}, L_{4}$, and the exceptional classes $P_{i}, Q_{i}, i=1, \ldots, 6$.

Proof. A dimension count shows that the moduli space in this case has dimension $4 \cdot 4-10=6$, so the Picard number of a very general $X$ is at most 14 . Let $\Lambda$ be the lattice spanned by the above classes. First, note that $\Lambda$ is already spanned by $L_{1}, \ldots, L_{4}$ and the ten classes $P_{i}, Q_{i}$, for $i=1, \ldots, 5$, since we may solve for $P_{6}$ and $Q_{6}$ from the above relations. Since these remaining fourteen classes are linearly independent (they have a nonsingular intersection matrix), they form a basis for $\Lambda$.

Let $Z_{i}=P_{i}-Q_{i}$. Computing the inverse of the Gram matrix shows that any element of the dual lattice has the form

$$
D=\frac{1}{2}\left(c_{1} L_{1}+c_{2} L_{2}\right)+\frac{1}{3} \sum_{i=1}^{5} d_{i} Z_{i}
$$


where $c_{i}$ and $d_{i}$ are integers. Suppose $D \in \overline{\mathrm{NS}}(X)$. Then $3 D \in \overline{\mathrm{NS}}(X)$, from which it follows that $D^{\prime}=\left(c_{1} L_{1}+c_{2} L_{2}\right) / 2 \in \overline{\mathrm{NS}}(X)$. We claim both $c_{1}$ and $c_{2}$ are even. If $c_{1}$ and $c_{2}$ are odd, then $D^{\prime 2}$ is odd, a contradiction. So at least one of $c_{1}$ and $c_{2}$ is even. If one is odd and one is even, we can find another divisor (by symmetry) with the parities reversed, and adding them will give us an element with both coefficients odd, a contradiction. Therefore, we may assume

$$
D=\frac{1}{3} \sum_{i=1}^{5} d_{i} Z_{i} .
$$

We may assume each $d_{i} \in\{-1,0,1\}$. But note that $Z_{i}^{2}=-6$ and $Z_{i} \cdot Z_{j}=0$ for $i \neq j$. Hence, $D^{2}=-2\left(\sum d_{i}^{2}\right) / 3$, and since this must be an (even) integer, we see that exactly three of the $d_{i}$ must be \pm 1 . Suppose without loss of generality that $\left(Z_{1}+Z_{2}+Z_{3}\right) / 3 \in \overline{\mathrm{NS}}(X)$. Then by symmetry any $\left(Z_{i}+Z_{j}+Z_{k}\right) / 3 \in \overline{\mathrm{NS}}(X)$. Therefore,

$$
\frac{1}{3}\left(Z_{1}+Z_{2}+Z_{3}\right)-\frac{1}{3}\left(Z_{1}+Z_{2}+Z_{4}\right)=\frac{1}{3}\left(Z_{3}-Z_{4}\right) \in \overline{\mathrm{NS}}(X),
$$

which is impossible, since exactly three of the $d_{i}$ are \pm 1 .

\subsection{Moduli problem. We now complete the proof of Theorem 9.1.}

Proof of Theorem 9.1. The above discussion describes how to construct a K3 surface lattice-polarized by $(\Lambda, S)$ from a triply symmetric penteract. It remains to show that from such data, the penteract $A$ constructed as in Section 7.3 is in fact triply symmetric. That is, starting from $X$ lattice-polarized by $(\Lambda, S)$, let $L_{1}, L_{2}$, $L_{3}$, and $L_{4}$ be the line bundles corresponding to the elements of $S$. Then we obtain a penteract $A \in \mathrm{H}^{0}\left(X, L_{1}\right) \otimes \mathrm{H}^{0}\left(X, L_{2}\right) \otimes \mathrm{H}^{0}\left(X, L_{3}\right) \otimes \mathrm{H}^{0}\left(X, L_{4}\right) \otimes(\operatorname{ker} \mu)^{\vee}$, where $\mu$ is the usual multiplication map on sections.

The rest of the proof builds on that of Theorem 8.1. In particular, that proof immediately shows that $A$ must be doubly symmetric, that is, symmetric in the fourth and fifth tensor factors. (Note that this argument relies on (8) with the exceptional fibers $P_{i}$.) By switching the roles of the indices 3 and 4 in that argument, and using the $Q_{i}$ for (8), we also see that $A$ is symmetric in the third and fifth factors. In other words, there are simultaneous identifications of the vector spaces $\mathrm{H}^{0}\left(X, L_{3}\right), \mathrm{H}^{0}\left(X, L_{4}\right)$, and $(\operatorname{ker} \mu)^{\vee}$ such that $A$ is triply symmetric in these three factors, that is, under these identifications, we may think of $A$ as an element of $\mathrm{H}^{0}\left(X, L_{1}\right) \otimes \mathrm{H}^{0}\left(X, L_{2}\right) \otimes \operatorname{Sym}^{3} \mathrm{H}^{0}\left(X, L_{3}\right)$.

9.3. Automorphisms. As in the previous penteract cases, we may again consider many automorphisms of the form $\alpha_{i j, k}$. All the 5-cycles meeting all five 
$X_{i j k l}$ are equivalent (up to reordering) to one of the following two:

$$
\begin{aligned}
& \Phi_{54123}: X_{1234} \rightarrow X_{1235} \rightarrow X_{2345} \rightarrow X_{1345} \rightarrow X_{1245} \rightarrow X_{1234} \\
& \Phi_{54132}: X_{1234} \rightarrow X_{1235} \rightarrow X_{2345} \rightarrow X_{1245} \rightarrow X_{1345} \rightarrow X_{1234} .
\end{aligned}
$$

Using the same techniques as in previous sections, namely, applying Lemma 7.2 and computing intersection numbers, we obtain the action of $\Phi_{54123}$ on the Néron-Severi lattice of the K3 surface $X_{\bar{F}}$ arising from a general triply symmetric penteract as the matrix

$$
\left(\begin{array}{cccccccccccccc}
-1 & 0 & 2 & 2 & 0 & 0 & 0 & 0 & 0 & 0 & 0 & 0 & 0 & 0 \\
-2 & 1 & 2 & 4 & 0 & 0 & 0 & 0 & 0 & 0 & 0 & 0 & 0 & 0 \\
0 & 0 & 0 & 1 & 0 & 0 & 0 & 0 & 0 & 0 & 0 & 0 & 0 & 0 \\
-1 & 1 & 1 & 1 & 0 & 0 & 0 & 0 & 0 & 0 & 0 & 0 & 0 & 0 \\
0 & 0 & 0 & 1 & 0 & -1 & 0 & 0 & 0 & 0 & 0 & 0 & 0 & 0 \\
-1 & 0 & 1 & 1 & 1 & 1 & 0 & 0 & 0 & 0 & 0 & 0 & 0 & 0 \\
0 & 0 & 0 & 1 & 0 & 0 & 0 & -1 & 0 & 0 & 0 & 0 & 0 & 0 \\
-1 & 0 & 1 & 1 & 0 & 0 & 1 & 1 & 0 & 0 & 0 & 0 & 0 & 0 \\
0 & 0 & 0 & 1 & 0 & 0 & 0 & 0 & 0 & -1 & 0 & 0 & 0 & 0 \\
-1 & 0 & 1 & 1 & 0 & 0 & 0 & 0 & 1 & 1 & 0 & 0 & 0 & 0 \\
0 & 0 & 0 & 1 & 0 & 0 & 0 & 0 & 0 & 0 & 0 & -1 & 0 & 0 \\
-1 & 0 & 1 & 1 & 0 & 0 & 0 & 0 & 0 & 0 & 1 & 1 & 0 & 0 \\
0 & 0 & 0 & 1 & 0 & 0 & 0 & 0 & 0 & 0 & 0 & 0 & 0 & -1 \\
-1 & 0 & 1 & 1 & 0 & 0 & 0 & 0 & 0 & 0 & 0 & 0 & 1 & 1
\end{array}\right) .
$$

The induced action of the other automorphism $\Phi_{54132}$ on the line bundles $L_{i}$ and the exceptional divisors $P_{j}$ is the same (up to reordering) as the action of $\Phi_{54321}$ on $L_{i}$ and $E_{j}$ in the doubly symmetric penteract case, and the induced action on the $Q_{j}$ may also be immediately computed.

\section{Doubly doubly symmetric penteracts: $2 \otimes \operatorname{Sym}^{2}(2) \otimes \operatorname{Sym}^{2}(2)$}

Suppose we have a penteract $A$ that is symmetric in the second and third coordinates, and also in the last two coordinates. Then we may use the theorems from Sections 7 and 8 to study the associated orbit problem. We prove that the general orbits of such tensors correspond to certain K3 surfaces with Picard rank at least 12 over $\bar{F}$ :

THEOREM 10.1. Let $V=V_{1} \otimes \operatorname{Sym}^{2} V_{2} \otimes \operatorname{Sym}^{2} V_{3}$ for 2-dimensional F-vector spaces $V_{1}, V_{2}, V_{3}$. Let $G^{\prime}$ be the group $\operatorname{GL}\left(V_{1}\right) \times \operatorname{GL}\left(V_{2}\right) \times \operatorname{GL}\left(V_{3}\right)$, and let $G$ be the quotient of $G^{\prime}$ by the kernel of the multiplication map $\mathbb{G}_{m} \times \mathbb{G}_{m} \times \mathbb{G}_{m} \rightarrow \mathbb{G}_{m}$ 
given by $\left(\gamma_{1}, \gamma_{2}, \gamma_{3}\right) \mapsto \gamma_{1} \gamma_{2}^{2} \gamma_{3}^{2}$. Let $\Lambda$ be the lattice whose Gram matrix is

$$
\left(\begin{array}{cccccccccccc}
0 & 2 & 2 & 2 & 0 & 0 & 0 & 0 & 0 & 0 & 0 & 0 \\
2 & 0 & 2 & 2 & 0 & 1 & 0 & 0 & 1 & 0 & 0 & 1 \\
2 & 2 & 0 & 2 & 0 & 1 & 0 & 0 & 1 & 0 & 0 & 1 \\
2 & 2 & 2 & 0 & 1 & 0 & 1 & 1 & 0 & 1 & 1 & 0 \\
0 & 0 & 0 & 1 & -2 & 1 & 0 & 0 & 0 & 0 & 0 & 0 \\
0 & 1 & 1 & 0 & 1 & -2 & 1 & 0 & 0 & 0 & 0 & 0 \\
0 & 0 & 0 & 1 & 0 & 1 & -2 & 0 & 0 & 0 & 0 & 0 \\
0 & 0 & 0 & 1 & 0 & 0 & 0 & -2 & 1 & 0 & 0 & 0 \\
0 & 1 & 1 & 0 & 0 & 0 & 0 & 1 & -2 & 1 & 0 & 0 \\
0 & 0 & 0 & 1 & 0 & 0 & 0 & 0 & 1 & -2 & 0 & 0 \\
0 & 0 & 0 & 1 & 0 & 0 & 0 & 0 & 0 & 0 & -2 & 1 \\
0 & 1 & 1 & 0 & 0 & 0 & 0 & 0 & 0 & 0 & 1 & -2
\end{array}\right),
$$

and let $S=\left\{e_{1}, e_{2}, e_{3}, e_{4}\right\}$. Then the $G(F)$-orbits of an open subset of $V(F)$ are in bijection with the $F$-points of an open subvariety of the moduli space $\mathcal{M}_{\Lambda, S}$ of $K 3$ surfaces $X$ lattice-polarized by $(\Lambda, S)$.

10.1. Néron-Severi lattice and moduli problem. In order to study the orbits of doubly doubly symmetric penteracts, we may use the geometric construction from Section 8, since an element of $V_{1} \otimes \mathrm{Sym}^{2} V_{2} \otimes \mathrm{Sym}^{2} V_{3}$ is also an element of $V_{1} \otimes V_{2} \otimes V_{2} \otimes \operatorname{Sym}^{2} V_{3}$ and $V_{1} \otimes \operatorname{Sym}^{2} V_{2} \otimes V_{3} \otimes V_{3}$. Thus, the K3 surfaces $X_{123}$ and $X_{145}$ each have at least six (rank) singularities over $\bar{F}$, and a numerical example shows us that they generically have exactly six singular points. Meanwhile, for a generic orbit, all of the other $X_{i j k}$ (namely, $X_{124}, X_{234}$ and $X_{345}$ ) are nonsingular, and the maps of the type $X_{1234} \rightarrow X_{123}$ blow up the six singular points.

The nonsingular K3's-which are all naturally isomorphic- thus contain two sets of six mutually nonintersecting lines, namely the exceptional fibers in $X_{124}$ coming from the blow-ups $X_{124} \stackrel{\sim}{\rightarrow} X_{1234} \rightarrow X_{123}$ and $X_{124} \stackrel{\sim}{\rightarrow} X_{1245} \rightarrow X_{145}$. Let $P_{i}$ and $Q_{i}$ for $1 \leqslant i \leqslant 6$ denote these exceptional fibers from $X_{123}$ and $X_{145}$, respectively. As explained below, each of the six lines in any one set intersects exactly two lines in the other set.

The map $X_{1234} \rightarrow \mathbb{P}\left(V_{1}^{\vee}\right)$ is a genus one fibration whose discriminant as a binary form on $V_{1}$ is of degree 24 and factors as the product of a fourth power of a cubic form times an irreducible degree twelve form. Thus the fibration has three reducible fibers of type $\mathrm{I}_{4}$, that is, each of these reducible fibers consists of four lines forming a 'rectangle'. Each set of six lines in the previous paragraph contains one pair of parallel lines from each of the three rectangles. That is, with choices of indices, each rectangle is made up of the lines corresponding to $P_{i}, Q_{i}$, $P_{i+1}$, and $Q_{i+1}$ for $i=1,3,5$. 
To explicitly see this correspondence among the twelve lines, we note that if $r_{0} \in \mathbb{P}\left(V_{1}^{\vee}\right)$ is a point giving a singular fiber in the genus one fibration, then it yields two rank singularities $\left(r_{0}, a, b\right)$ and $\left(r_{0}, b, a\right)$ on $X_{123}$. The map $X_{124} \rightarrow$ $X_{123}$ blows up these singularities to the lines $\left(r_{0}, a, *\right)$ and $\left(r_{0}, b, *\right)$, where we use $*$ to mean that the coordinate in $\mathbb{P}\left(V_{3}^{\vee}\right)$ may vary freely. Similarly, each such $r_{0}$ gives two rank singularities $\left(r_{0}, c, d\right)$ and $\left(r_{0}, d, c\right)$ on $X_{145}$, and under $X_{124} \rightarrow$ $X_{145}$, these blow up to lines $\left(r_{0}, *, c\right)$ and $\left(r_{0}, *, d\right)$ for any $* \in \mathbb{P}\left(V_{2}^{\vee}\right)$. Therefore, the latter two lines each intersect each of the former two lines in a single point, giving the four intersection points $\left(r_{0}, a, c\right),\left(r_{0}, a, d\right),\left(r_{0}, b, d\right)$, and $\left(r_{0}, b, c\right)$ in $X_{124}$.

The usual line bundles $L_{i}$ for $1 \leqslant i \leqslant 4$, given as the pullbacks of $\mathcal{O}_{\mathbb{P}\left(V_{i}^{\vee}\right)}(1)$ to $X_{1234}$, satisfy:

$$
L_{1}+L_{2}+L_{3}=2 L_{4}+\sum_{i} P_{i}
$$

and

$$
2 L_{1}-L_{2}-L_{3}+2 L_{4}=\sum_{i} Q_{i}
$$

Of course, (22) is clear from Lemma 7.2, and the second relation (23) comes from repeated applications of that lemma along each step of the composition $X_{1234} \rightarrow$ $X_{124} \rightarrow X_{1245} \rightarrow X_{145}$.

To determine the Néron-Severi lattice (over $\bar{F}$ ) of the K3 surface associated to a generic doubly doubly symmetric penteract, we use the explicit geometry and the relations described above in (22) and (23) to compute the intersection numbers between the divisor classes. The only nonzero intersection numbers are as follows:

$$
\begin{array}{rlrl}
L_{i} \cdot L_{j} & =2 \text { for } i \neq j, & L_{2} \cdot Q_{j}=L_{3} \cdot Q_{j}=1, \\
P_{i}^{2} & =Q_{i}^{2}=-2, & L_{4} \cdot P_{j}=1, & \\
P_{i} \cdot Q_{i} & =P_{i} \cdot Q_{i+1}=P_{i+1} \cdot Q_{i+1}=P_{i+1} \cdot Q_{i}=1 \quad \text { for } i \in\{1,3,5\} .
\end{array}
$$

The rank of the intersection matrix is 12 , and the span of the divisors is a sublattice of $\overline{\mathrm{NS}}(X)$ of discriminant 256. A basis for the Néron-Severi lattice is $\left\{L_{1}, L_{2}, L_{4}\right.$, $\left.P_{1}, Q_{1}, P_{2}, P_{3}, Q_{3}, P_{4}, P_{5}, Q_{5}, P_{6}\right\}$, and the corresponding Gram matrix is given by (21).

PROPOSITION 10.2. For a generic $X$ in this family of $K 3$ surfaces, $\overline{\mathrm{NS}}(X)$ is spanned over $\mathbb{Z}$ by the $L_{i}$ for $i=1, \ldots, 4$ and the exceptional classes $P_{i}$ and $Q_{i}$ for $i=1, \ldots, 6$. 
Proof. The moduli space of these K3s has dimension $2 \cdot 3 \cdot 3-10=8$, so the Picard number of the generic surface in this space is at most 12. Let $\Lambda$ be the rank-12 lattice spanned by the above divisors (equivalently, with basis $\left\{L_{1}, L_{2}\right.$, $\left.\left.L_{4}, P_{1}, Q_{1}, P_{2}, P_{3}, Q_{3}, P_{4}, P_{5}, Q_{5}, P_{6}\right\}\right)$. Since the discriminant of $\Lambda$ is 256 , it is enough to show that it is 2-saturated. Suppose a divisor class

$$
D=\alpha_{1} L_{1}+\alpha_{2} L_{2}+\alpha_{4} L_{4}+\sum_{i=1}^{6} \beta_{i} P_{i}+\gamma_{1} Q_{1}+\gamma_{3} Q_{3}+\gamma_{5} Q_{5}
$$

is in $\overline{\mathrm{NS}}(X)$ for some collection of rational numbers $\alpha_{i}, \beta_{j}, \gamma_{k}$ whose denominators are powers of 2 . Then by symmetry, so is the divisor $D^{\prime}$ obtained by replacing $L_{2}$ by $L_{3}$. Then $D-D^{\prime}=\alpha_{2}\left(L_{2}-L_{3}\right) \in \overline{\mathrm{NS}}(X)$, which forces $\alpha_{2}$ to be an integer, since the self-intersection $-4 \alpha_{2}^{2}$ of this divisor must be an even integer. So we may assume $\alpha_{2}=0$, and similarly, $\alpha_{4}=0$. Therefore, $D$ has the form

$$
D=\alpha_{1} L_{1}+\sum_{i=1}^{6} \beta_{i} P_{i}+\gamma_{1} Q_{1}+\gamma_{3} Q_{3}+\gamma_{5} Q_{5} .
$$

Again by symmetry, the divisor $D^{\prime \prime}=\alpha_{1} L_{1}+\sum_{i=1}^{6} \beta_{i} P_{i}+\gamma_{1} Q_{2}+\gamma_{3} Q_{4}+\gamma_{5} Q_{6}$ is also in $\overline{\mathrm{NS}}(X)$. Then $D-D^{\prime \prime} \in \overline{\mathrm{NS}}(X)$ forces all the $\gamma_{i}$ to vanish, by Lemma 2.3 (since the $Q_{i}$ are all disjoint (-2)-curves). Hence $D=\alpha_{1} L_{1}+\sum_{i=1}^{6} \beta_{i} P_{i}$. Then $D^{\prime \prime \prime}=\alpha_{1} L_{1}+\sum_{i=1}^{4} \beta_{i} P_{i}+\beta_{6} P_{5}+\beta_{5} P_{6}$ is also in $\overline{\mathrm{NS}}(X)$ by symmetry, and considering $D-D^{\prime \prime \prime}$ shows that $\beta_{5}$ and $\beta_{6}$ are equal modulo $\mathbb{Z}$. Similar symmetry arguments force all the $\beta_{i}$ to be equal to each other. Hence $D=\alpha L_{1}+\beta\left(\sum P_{i}\right)$. Then $D^{2}=-6 \beta^{2}$ is an even integer, which forces $\beta \in \mathbb{Z}$. Subtracting $\beta\left(\sum P_{i}\right) \in$ $\Lambda$, we may assume $\alpha L_{1} \in \overline{\mathrm{NS}}(X)$. In fact, this forces $\alpha \in \mathbb{Z}$ as well, since $L_{1}$ is the class of an elliptic fiber and cannot be a nontrivial multiple of another divisor.

We now complete the proof of Theorem 10.1.

Proof of Theorem 10.1. The above geometric constructions explain how to obtain a $(\Lambda, S)$-polarized $\mathrm{K} 3$ surface from a doubly doubly symmetric penteract. On the other hand, given such a K3 $X$, let $L_{1}, L_{2}, L_{3}$, and $L_{4}$ be line bundles corresponding to the elements of $S$. Then we may use these line bundles as in Section 7.3 to produce a penteract $A \in \mathrm{H}^{0}\left(X, L_{1}\right) \otimes \mathrm{H}^{0}\left(X, L_{2}\right) \otimes \mathrm{H}^{0}(X$, $\left.L_{3}\right) \otimes \mathrm{H}^{0}\left(X, L_{4}\right) \otimes(\operatorname{ker} \mu)^{\vee}$, where $\mu$ is the usual multiplication map; we now show that it has the appropriate symmetry.

By the argument in the proof of Theorem 8.1, and using Lemma 7.2, we immediately see that there is an identification of $\mathrm{H}^{0}\left(X, L_{4}\right)$ and $(\operatorname{ker} \mu)^{\vee}$ such that $A$ is doubly symmetric in those coordinates. 
Similarly, we may switch the roles of the indices 2 and 3 with those of 4 and 5 , respectively, to obtain the second symmetry. For example, we may use $A$ to construct K3 surfaces $X_{1245}$ and $X_{145}$ (with the divisor classes in $\Lambda$ ). The line bundles $M_{i}$ coming from pulling back $\mathcal{O}_{\mathbb{P}\left(V_{i}\right)^{\vee}}(1)$ to $X_{1245}$ via projection for $i=1$, 2, 4, 5 may be used to make another penteract $B$, which is $\mathrm{GL}_{2}^{5}$-equivalent to $A$ (by the proof of Theorem 7.1). (Note that in fact $M_{i}$ and $L_{i}$ are isomorphic for $i=1$, 2,4 .) Then the same argument as in Theorem 8.1 shows that the line bundle $M_{2}$ is isomorphic to the line bundle $M_{3}^{(145)}$, and in fact, the corresponding vector spaces may be identified so that $B$ is symmetric in those two directions.

Therefore, via the above identifications of vector spaces, our penteract $A$ may be viewed as an element of the tensor space $\mathrm{H}^{0}\left(X, L_{1}\right) \otimes \operatorname{Sym}^{2} \mathrm{H}^{0}(X$, $\left.L_{2}\right) \otimes \operatorname{Sym}^{2} \mathrm{H}^{0}\left(X, L_{4}\right)$, as desired.

10.2. Automorphisms. We may again consider the automorphisms $\alpha_{i j, k}$ for doubly doubly symmetric penteracts. By symmetry, to understand the 5-cycles passing through all five $X_{i j k l}$, which are all compositions of three $\alpha_{i j, k}$ 's, it suffices to understand the following three:

$$
\begin{aligned}
& \Phi_{53214}: X_{1234} \rightarrow X_{1245} \rightarrow X_{1345} \rightarrow X_{2345} \rightarrow X_{1235} \rightarrow X_{1234} \\
& \Phi_{53421}: X_{1234} \rightarrow X_{1245} \rightarrow X_{1235} \rightarrow X_{1345} \rightarrow X_{2345} \rightarrow X_{1234} \\
& \text { or } \Phi_{53241}: X_{1234} \rightarrow X_{1245} \rightarrow X_{1345} \rightarrow X_{1235} \rightarrow X_{2345} \rightarrow X_{1234}
\end{aligned}
$$

We first study the 5-cycle $\Phi_{54321}$. Applying Lemma 7.2 and determining intersection numbers, we compute the action of the automorphism $\Phi_{54321}$ on $\overline{\mathrm{NS}}(X)$ arising from a general doubly doubly symmetric penteract as the matrix

$$
\left(\begin{array}{cccccccccccc}
1 & 4 & -2 & 2 & 0 & 0 & 0 & 0 & 0 & 0 & 0 & 0 \\
0 & 2 & -1 & 2 & 0 & 0 & 0 & 0 & 0 & 0 & 0 & 0 \\
0 & 1 & 0 & 0 & 0 & 0 & 0 & 0 & 0 & 0 & 0 & 0 \\
1 & 1 & -1 & 1 & 0 & 0 & 0 & 0 & 0 & 0 & 0 & 0 \\
0 & 1 & 0 & 0 & -1 & 0 & 0 & 0 & 0 & 0 & 0 & 0 \\
1 & 1 & -1 & 1 & 0 & -1 & 0 & 0 & 0 & 0 & 0 & 0 \\
0 & 1 & 0 & 0 & 0 & 0 & -1 & 0 & 0 & 0 & 0 & 0 \\
0 & 1 & 0 & 0 & 0 & 0 & 0 & -1 & 0 & 0 & 0 & 0 \\
1 & 1 & -1 & 1 & 0 & 0 & 0 & 0 & -1 & 0 & 0 & 0 \\
0 & 1 & 0 & 0 & 0 & 0 & 0 & 0 & 0 & -1 & 0 & 0 \\
0 & 1 & 0 & 0 & 0 & 0 & 0 & 0 & 0 & 0 & -1 & 0 \\
1 & 1 & -1 & 1 & 0 & 0 & 0 & 0 & 0 & 0 & 0 & -1
\end{array}\right)
$$

with respect to the basis $\left\{L_{1}, L_{2}, L_{3}, L_{4}, P_{1}, Q_{1}, P_{2}, P_{3}, Q_{3}, P_{4}, P_{5}, Q_{5}\right\}$. 
The induced action of the automorphism $\Phi_{53421}$ on the $L_{i}$ is the same (up to reordering) as in the usual penteract case (and it is thus simple to compute the action on the exceptional divisors). For the automorphism $\Phi_{53241}$, the induced action on the $L_{i}$ and the exceptional divisors $Q_{j}$ are the same (up to reordering) as the induced action of $\Phi_{54321}$ in the doubly symmetric penteract case.

\section{Doubly triply symmetric penteracts: $\operatorname{Sym}^{2}(2) \otimes \operatorname{Sym}^{3}(2)$}

We now study penteracts that are symmetric in the first two coordinates and also symmetric in the last three coordinates. We prove that the orbits of the space of doubly triply symmetric penteracts are related to certain K3 surfaces with Néron-Severi rank at least 15 over $\bar{F}$. Note that these penteracts also have an interpretation as bidegree $(2,3)$ curves in $\mathbb{P}^{1} \times \mathbb{P}^{1}$, which can be used to connect the moduli space below with the universal Picard scheme $\operatorname{Pic}_{\mathcal{M}_{2}}^{1}$ over the moduli space of genus 2 curves.

THEOREM 11.1. Let $V=\operatorname{Sym}^{2} V_{1} \otimes \mathrm{Sym}^{3} V_{2}$ for 2-dimensional $F$-vector spaces $V_{1}$ and $V_{2}$. Let $G^{\prime}$ be the group $\mathbb{G}_{m} \times \operatorname{GL}\left(V_{1}\right) \times \mathrm{GL}\left(V_{2}\right)$, and let $G$ be the quotient of $G^{\prime}$ by the kernel of the multiplication map $\mathbb{G}_{m} \times \mathbb{G}_{m} \times \mathbb{G}_{m} \rightarrow \mathbb{G}_{m}$ given by $\left(\gamma_{1}, \gamma_{2}, \gamma_{3}\right) \mapsto \gamma_{1} \gamma_{2}^{2} \gamma_{3}^{3}$. Let $\Lambda$ be the lattice whose Gram matrix is

$$
\left(\begin{array}{ccccccccccccccc}
0 & 2 & 2 & 2 & 0 & 0 & 0 & 0 & 0 & 0 & 0 & 0 & 0 & 0 & 1 \\
2 & 0 & 2 & 2 & 0 & 0 & 0 & 0 & 0 & 0 & 0 & 0 & 0 & 0 & 1 \\
2 & 2 & 0 & 2 & 0 & 1 & 0 & 1 & 0 & 1 & 0 & 1 & 0 & 1 & 0 \\
2 & 2 & 2 & 0 & 1 & 0 & 1 & 0 & 1 & 0 & 1 & 0 & 1 & 0 & 0 \\
0 & 0 & 0 & 1 & -2 & 1 & 0 & 0 & 0 & 0 & 0 & 0 & 0 & 0 & 1 \\
0 & 0 & 1 & 0 & 1 & -2 & 0 & 0 & 0 & 0 & 0 & 0 & 0 & 0 & 0 \\
0 & 0 & 0 & 1 & 0 & 0 & -2 & 1 & 0 & 0 & 0 & 0 & 0 & 0 & 1 \\
0 & 0 & 1 & 0 & 0 & 0 & 1 & -2 & 0 & 0 & 0 & 0 & 0 & 0 & 0 \\
0 & 0 & 0 & 1 & 0 & 0 & 0 & 0 & -2 & 1 & 0 & 0 & 0 & 0 & 0 \\
0 & 0 & 1 & 0 & 0 & 0 & 0 & 0 & 1 & -2 & 0 & 0 & 0 & 0 & 1 \\
0 & 0 & 0 & 1 & 0 & 0 & 0 & 0 & 0 & 0 & -2 & 1 & 0 & 0 & 0 \\
0 & 0 & 1 & 0 & 0 & 0 & 0 & 0 & 0 & 0 & 1 & -2 & 0 & 0 & 1 \\
0 & 0 & 0 & 1 & 0 & 0 & 0 & 0 & 0 & 0 & 0 & 0 & -2 & 1 & 0 \\
0 & 0 & 1 & 0 & 0 & 0 & 0 & 0 & 0 & 0 & 0 & 0 & 1 & -2 & 0 \\
1 & 1 & 0 & 0 & 1 & 0 & 1 & 0 & 0 & 1 & 0 & 1 & 0 & 0 & -2
\end{array}\right)
$$

and let $S=\left\{e_{1}, e_{2}, e_{3}, e_{4}\right\}$. Then the $G(F)$-orbits of an open subset of $V(F)$ are in bijection with the $F$-points of an open subvariety of the moduli space $\mathcal{M}_{\Lambda, S}$ of $K 3$ surfaces $X$ lattice-polarized by $(\Lambda, S)$. 
11.1. Néron-Severi lattice and moduli problem. By exploiting the double symmetry in the first two coordinates and triple symmetry in the last three coordinates, we may apply the constructions in all of the previous penteract sections! That is, the space $\mathrm{Sym}^{2} V_{1} \otimes \mathrm{Sym}^{3} V_{2}$ is a subspace of doubly symmetric penteracts $V_{1} \otimes V_{1} \otimes V_{2} \otimes \mathrm{Sym}^{2} V_{2}$ or $V_{2} \otimes V_{2} \otimes V_{2} \otimes \operatorname{Sym}^{2} V_{1}$, triply symmetric penteracts $V_{1} \otimes V_{1} \otimes \operatorname{Sym}^{3} V_{2}$, and doubly doubly symmetric penteracts $V_{2} \otimes$ $\mathrm{Sym}^{2} V_{1} \otimes \operatorname{Sym}^{2} V_{2}$.

Given an element of $\operatorname{Sym}^{2} V_{1} \otimes \operatorname{Sym}^{3} V_{2}$, using the usual notation, we construct K3 surfaces $X_{123}\left(=X_{124}=X_{125}\right)$ and $X_{345}$ that have at least six rank singularities (over $\bar{F}$ ). A numerical example shows us that generically there are exactly six singularities on each of $X_{123}$ and $X_{345}$. Meanwhile, the other K3 surfaces ( $X_{134}$, $X_{1234}$, and $\left.X_{1345}\right)$ are generically nonsingular and isomorphic, and the maps $X_{134} \rightarrow X_{1234} \rightarrow X_{123}, X_{134} \rightarrow X_{1234} \rightarrow X_{124}$, and $X_{134} \rightarrow X_{1345} \rightarrow X_{345}$ blow down sets of six lines to each of the corresponding sets of six singular points. The nonsingular K3 surface $X_{1234}$ contains at least three sets of six lines; call the divisors corresponding to these lines $P_{i}, Q_{i}$, and $E_{i}$, respectively, for $1 \leqslant i \leqslant 6$. Their intersection numbers are computed below, by using the various genus one fibrations.

Either projection map from $X_{1234}$ to $\mathbb{P}\left(V_{1}^{\vee}\right)$ is a genus one fibration whose discriminant is a degree 24 binary form on $V_{1}$ that factors as the cube of a degree six form and an irreducible degree six form (as a special case of the triply symmetric penteract). Thus the fibration has six reducible fibers of type $\mathrm{I}_{3}$. Each of these reducible fibers consists of three lines in a 'triangle'; in each of the six triangles, there are two distinguished lines that correspond to $P_{i}$ and $Q_{i}$, respectively, for $1 \leqslant i \leqslant 6$.

Either projection map from $X_{1234}$ to $\mathbb{P}\left(V_{2}^{\vee}\right)$ is a genus one fibration with three reducible fibers of type $\mathrm{I}_{4}$ (as a special case of the doubly doubly symmetric penteract). That is, each of the three reducible fibers is a rectangle, and two of the opposite sides of each rectangle are the 6 lines $E_{i}$ from the 18 described above. The other two parallel sides in each rectangle are given by $P_{2 j-1}$ and $P_{2 j}$ for $1 \leqslant j \leqslant 3$, for the projection $\pi_{3}$ to the third factor of $\mathbb{P}^{1}$; for the projection $\pi_{4}$, we get a similar picture, but with the $P$ 's replaced by the $Q$ 's.

We explicitly list these 18 lines in $X_{1234}$. For $1 \leqslant j \leqslant 3$, let $\left(r_{j}, s_{j}\right) \in \mathbb{P}\left(V_{1}^{\vee}\right) \times$ $\mathbb{P}\left(V_{2}^{\vee}\right)$ be distinct images of singular points on $X_{123}$ under the projection $\pi_{12}$ to $\mathbb{P}\left(V_{1}^{\vee}\right) \times \mathbb{P}\left(V_{1}^{\vee}\right)$, where $r_{j} \neq s_{k}$ for any $1 \leqslant j, k \leqslant 3$. Then the other singular points will project to $\left(s_{j}, r_{j}\right)$ for $1 \leqslant j \leqslant 3$, so the six singular points on $X_{123}$ will be, for $1 \leqslant j \leqslant 3$, given by $\left(r_{j}, s_{j}, t_{j}\right)$ and $\left(s_{j}, r_{j}, t_{j}\right)$ for some $t_{j} \in \mathbb{P}\left(V_{2}^{\vee}\right)$. Thus, we obtain four lines in $X_{1234}$ for each $j$ :

$$
\begin{array}{rlrl}
P_{2 j-1} & =\left(r_{j}, s_{j}, t_{j}, *\right) & & Q_{2 j-1}=\left(r_{j}, s_{j}, *, t_{j}\right), \\
P_{2 j} & =\left(s_{j}, r_{j}, t_{j}, *\right) & Q_{2 j}=\left(s_{j}, r_{j}, *, t_{j}\right),
\end{array}
$$


where we again use $*$ to mean that the coordinate varies freely in the appropriate $\mathbb{P}^{1}$. These are the six pairs of lines in the reducible $\mathrm{I}_{2}$ fibers in the projection $X_{1234} \rightarrow \mathbb{P}\left(V_{1}^{\vee}\right)$, and it is clear that they are the blow-ups of the singular points from $X_{123}$ and $X_{124}$.

Using these explicit points in projective space, we also see that the six singular points in $X_{345}$ are just the points $\left(t_{i}, t_{j}, t_{k}\right)$ for the permutations $\{i, j, k\}$ of $\{1,2,3\}$. Therefore, the surface $X_{1234}$ contains six lines of the form

$$
E_{i}=\left(*, \diamond, t_{j}, t_{k}\right), \quad E_{i+3}=\left(*, \diamond, t_{k}, t_{j}\right)
$$

for $(i, j, k)$ ranging over cyclic permutations of $(1,2,3)$, where $*$ and $\diamond$ are varying coordinates connected by a $(1,1)$ equation. These are the six lines $E_{i}$ described above.

The line bundles $L_{i}$ obtained from pulling back $\mathcal{O}_{\mathbb{P}\left(V_{i}^{\vee}\right)}(1)$ to $X_{1234}$, for $1 \leqslant i \leqslant$ 4 , satisfy the following relations with the exceptional lines:

$$
\begin{aligned}
& L_{1}+L_{2}+L_{3}=2 L_{4}+\sum_{i=1}^{6} P_{i}, \\
& L_{1}+L_{2}+L_{4}=2 L_{3}+\sum_{i=1}^{6} Q_{i},
\end{aligned}
$$

and

$$
-L_{1}-L_{2}+2 L_{3}+2 L_{4}=\sum_{i=1}^{6} E_{i} .
$$

These are obtained from repeated applications of Lemma 7.2. The nonzero intersections between all these divisors are as follows:

$$
\begin{array}{rrr}
L_{i} \cdot L_{j}=2 \text { for } i \neq j, & L_{1} \cdot E_{i}=L_{2} \cdot E_{i}=1, & \\
L_{3} \cdot Q_{i}=1, & L_{4} \cdot P_{i}=1, & P_{i} \cdot Q_{i}=1, \\
P_{1}, P_{2} \text { intersect } E_{3}, E_{5}, & P_{3}, P_{4} \text { intersect } E_{1}, E_{6}, & P_{5}, P_{6} \text { intersect } E_{2}, E_{4}, \\
Q_{1}, Q_{2} \text { intersect } E_{2}, E_{6}, & Q_{3}, Q_{4} \text { intersect } E_{3}, E_{4}, & Q_{5}, Q_{6} \text { intersect } E_{1}, E_{5},
\end{array}
$$

where 'intersect' means has intersection number 1 . As a consequence, the NéronSeveri lattice of $\left(X_{1234}\right)_{\bar{F}}$ has rank 15 and discriminant 108. A basis for the lattice consists of the divisors $L_{1}, L_{2}, L_{3}, L_{4}, P_{1}, Q_{1}, \ldots, P_{5}, Q_{5}, E_{3}$, and the corresponding Gram matrix is (25).

PROPOSITION 11.2. For a very general $X$ in this family of $K 3$ surfaces, $\overline{\mathrm{NS}}(X)$ is spanned over $\mathbb{Z}$ by $L_{i}, i=1, \ldots, 4$, and the exceptional classes $P_{i}, Q_{i}, E_{i}$, $i=1, \ldots, 6$. 
Proof. Let $\Lambda$ be the rank-15 lattice spanned by the $L_{i}, P_{i}, Q_{i}, E_{i}$ as in the statement of the proposition. Consider the elliptic fibration $\pi: X \rightarrow \mathbb{P}\left(V_{1}^{\vee}\right)$. Taking the class of the zero section to be

$$
O=-L_{1}-L_{2}+2 P_{1}+Q_{1}+2 P_{2}+Q_{2}+P_{3}+2 Q_{3}+P_{4}+2 Q_{4}+3 E_{3},
$$

we see that $\left\{P_{1}, Q_{1}\right\}, \ldots,\left\{P_{6}, Q_{6}\right\}$ give the nonidentity components of the six $\mathrm{I}_{3}$ fibers. The curve $E_{1}$ gives a section $Q$ of height $4 / 3$, whereas the class of $L_{3}-L_{2}+L_{1}+O$ is a 3-torsion section $T$. The discriminant of the lattice spanned by these sections and the components of the fibers is $4 / 3 \cdot 3^{6} / 9=108$, so it is all of $\Lambda$. We must now show that generically, $\Lambda$ is all of $\overline{\mathrm{NS}}(X)$.

For a very general $X$ in this family of $\mathrm{K} 3$ surfaces, the rank of $\overline{\mathrm{NS}}(X)$ is at most 15 , since the dimension of the moduli space is $3 \cdot 4-7=5$. Since the discriminant of $\Lambda$ is $108=2^{2} \cdot 3^{3}$, we just need to check that $\Lambda$ is 2 - and 3-saturated. We observe from the configuration of fibers that there cannot be a 2-torsion section, and that $Q$ cannot be twice a section $Q^{\prime}$, since this would force the height of $Q^{\prime}$ to be $1 / 3$, which is impossible. This checks 2-saturation. For similar reasons, $Q$ cannot be thrice a point, and there cannot be a 9-torsion point. So we just need to check that the elliptic surface does not have full 3-torsion. Observe that if $T^{\prime}$ were another 3-torsion section, independent of $T$ over $\mathbb{F}_{3}$, then to have height $0=4-6(2 / 3)$, both $T$ and $T^{\prime}$ must intersect nonidentity components of each of the six $\mathrm{I}_{3}$ fibers. But then at least one $T+T^{\prime}$ or $T-T^{\prime}$ cannot satisfy the same property, and yet it is a 3-torsion point. This gives a contradiction.

We now complete the proof of Theorem 11.1.

Proof of Theorem 11.1. The above discussion explains how to construct a K3 surface lattice-polarized by $(\Lambda, S)$ from a doubly triply symmetric penteract. Given such a K3 surface $X$, let $L_{1}, L_{2}, L_{3}$, and $L_{4}$ be line bundles on $X$ corresponding to the elements of $S$. As in the previous cases, we use the construction from Section 7.3 to build a penteract $A \in \mathrm{H}^{0}\left(X, L_{1}\right) \otimes \mathrm{H}^{0}(X$, $\left.L_{2}\right) \otimes \mathrm{H}^{0}\left(X, L_{3}\right) \otimes \mathrm{H}^{0}\left(X, L_{4}\right) \otimes(\operatorname{ker} \mu)^{\vee}$. The proof of Theorem 9.1 shows that there is a simultaneous identification of $\mathrm{H}^{0}\left(X, L_{3}\right), \mathrm{H}^{0}\left(X, L_{4}\right)$, and $(\operatorname{ker} \mu)^{\vee}$ that shows that $A$ is triply symmetric with respect to those coordinates, that is, we can think of $A$ as an element of $\mathrm{H}^{0}\left(X, L_{1}\right) \otimes \mathrm{H}^{0}\left(X, L_{2}\right) \otimes \operatorname{Sym}^{3} \mathrm{H}^{0}\left(X, L_{3}\right)$.

To show the last symmetry, we use an argument similar to that in the proof of Theorem 10.1. That is, we switch the roles of the indices 1 and 2 with 4 and 5 and apply the proof of Theorem 8.1. Using the intersection matrix (25), combined with Lemma 7.2, shows that the vector spaces $\mathrm{H}^{0}\left(X, L_{1}\right)$ and $\mathrm{H}^{0}\left(X, L_{2}\right)$ may be identified and $A$ is symmetric in those two coordinates.

Thus, we obtain a penteract $A$ in the space $\operatorname{Sym}^{2} \mathrm{H}^{0}\left(X, L_{1}\right) \otimes \operatorname{Sym}^{3} \mathrm{H}^{0}\left(X, L_{3}\right)$, as desired. 
11.2. Automorphisms. The automorphisms $\alpha_{i j, k}$ again arise for doubly triply symmetric penteracts. The 5-cycles through all five $X_{i j k l}$ are all equivalent to either

$$
\begin{aligned}
\Phi_{53214}: X_{1234} \rightarrow X_{1245} \rightarrow X_{1345} \rightarrow X_{2345} \rightarrow X_{1235} \rightarrow X_{1234} \\
\text { or } \Phi_{52413}: X_{1234} \rightarrow X_{1345} \rightarrow X_{1235} \rightarrow X_{2345} \rightarrow X_{1245} \rightarrow X_{1234}
\end{aligned}
$$

Applying Lemma 7.2 and computing intersection numbers, we find that the action of the first automorphism $\Phi_{53214}$ on $\overline{\mathrm{NS}}(X)$ arising from a general doubly triply symmetric penteract is given by the matrix

$$
\left(\begin{array}{ccccccccccccccc}
2 & -1 & 0 & 2 & 0 & 0 & 0 & 0 & 0 & 0 & 0 & 0 & 0 & 0 & 0 \\
1 & 0 & 0 & 0 & 0 & 0 & 0 & 0 & 0 & 0 & 0 & 0 & 0 & 0 & 0 \\
0 & 0 & 0 & 1 & 0 & 0 & 0 & 0 & 0 & 0 & 0 & 0 & 0 & 0 & 0 \\
1 & -1 & 1 & 1 & 0 & 0 & 0 & 0 & 0 & 0 & 0 & 0 & 0 & 0 & 0 \\
0 & 0 & 0 & 0 & 0 & 1 & 0 & 0 & 0 & 0 & 0 & 0 & 0 & 0 & 0 \\
1 & 0 & 0 & 0 & -1 & -1 & 0 & 0 & 0 & 0 & 0 & 0 & 0 & 0 & 0 \\
0 & 0 & 0 & 0 & 0 & 0 & 0 & 1 & 0 & 0 & 0 & 0 & 0 & 0 & 0 \\
1 & 0 & 0 & 0 & 0 & 0 & -1 & -1 & 0 & 0 & 0 & 0 & 0 & 0 & 0 \\
0 & 0 & 0 & 0 & 0 & 0 & 0 & 0 & 0 & 1 & 0 & 0 & 0 & 0 & 0 \\
1 & 0 & 0 & 0 & 0 & 0 & 0 & 0 & -1 & -1 & 0 & 0 & 0 & 0 & 0 \\
0 & 0 & 0 & 0 & 0 & 0 & 0 & 0 & 0 & 0 & 0 & 1 & 0 & 0 & 0 \\
1 & 0 & 0 & 0 & 0 & 0 & 0 & 0 & 0 & 0 & -1 & -1 & 0 & 0 & 0 \\
0 & 0 & 0 & 0 & 0 & 0 & 0 & 0 & 0 & 0 & 0 & 0 & 0 & 1 & 0 \\
1 & 0 & 0 & 0 & 0 & 0 & 0 & 0 & 0 & 0 & 0 & 0 & -1 & -1 & 0 \\
-1 & -1 & 0 & 1 & 1 & 0 & 1 & 0 & 1 & 1 & 1 & 1 & 0 & 0 & 1
\end{array}\right)
$$

with respect to the basis $\left\{L_{1}, L_{2}, L_{3}, L_{4}, P_{1}, Q_{1}, \ldots, P_{5}, Q_{5}, E_{3}\right\}$. Also, the induced action of the 5-cycle $\Phi_{52413}$ on the $L_{i}$ and the $Q_{j}$ is the same (up to reordering) as the induced action of $\Phi_{54321}$ in the doubly symmetric penteract case, and as in previous cases, expressions for the divisors $\Phi_{52413}^{*} P_{j}$ and $\Phi_{52413}^{*} E_{j}$ follow immediately. These two types of 5-cycles will be shown in Section 17.4 to be fixed-point-free in general and have positive entropy.

\section{Quadruply symmetric penteracts: $2 \otimes \operatorname{Sym}^{4}(2)$}

Suppose we now have a penteract that is symmetric in the last four coordinates; we will show that such penteracts give rise to K3 surfaces with Néron-Severi rank at least 17 over $\bar{F}$. Note that such tensors may also be viewed as pencils of binary quartic forms, whose invariant theory was worked out in [72]. 
THEOREM 12.1. Let $V=V_{1} \otimes \operatorname{Sym}^{4} V_{2}$ for 2-dimensional $F$-vector spaces $V_{1}$ and $V_{2}$. Let $G^{\prime}$ be the group $\mathrm{GL}\left(V_{1}\right) \times \mathrm{GL}\left(V_{2}\right)$, and let $G$ be the quotient of $G^{\prime}$ by the kernel of the multiplication map $\mathbb{G}_{m} \times \mathbb{G}_{m} \rightarrow \mathbb{G}_{m}$ given by $\left(\gamma_{1}, \gamma_{2}\right) \mapsto \gamma_{1} \gamma_{2}^{4}$. Let $\Lambda$ be the lattice whose Gram matrix is

$\left(\begin{array}{ccccccccccccccccc}0 & 2 & 2 & 2 & 0 & 0 & 0 & 0 & 0 & 0 & 0 & 0 & 0 & 0 & 0 & 0 & 0 \\ 2 & 0 & 2 & 2 & 1 & 0 & 0 & 1 & 0 & 1 & 0 & 0 & 1 & 0 & 1 & 0 & 0 \\ 2 & 2 & 0 & 2 & 0 & 1 & 0 & 0 & 1 & 0 & 1 & 0 & 0 & 1 & 0 & 1 & 0 \\ 2 & 2 & 2 & 0 & 0 & 0 & 1 & 0 & 0 & 0 & 0 & 1 & 0 & 0 & 0 & 0 & 1 \\ 0 & 1 & 0 & 0 & -2 & 1 & 0 & 0 & 0 & 0 & 0 & 0 & 0 & 0 & 0 & 0 & 0 \\ 0 & 0 & 1 & 0 & 1 & -2 & 1 & 0 & 0 & 0 & 0 & 0 & 0 & 0 & 0 & 0 & 0 \\ 0 & 0 & 0 & 1 & 0 & 1 & -2 & 1 & 0 & 0 & 0 & 0 & 0 & 0 & 0 & 0 & 0 \\ 0 & 1 & 0 & 0 & 0 & 0 & 1 & -2 & 1 & 0 & 0 & 0 & 0 & 0 & 0 & 0 & 0 \\ 0 & 0 & 1 & 0 & 0 & 0 & 0 & 1 & -2 & 0 & 0 & 0 & 0 & 0 & 0 & 0 & 0 \\ 0 & 1 & 0 & 0 & 0 & 0 & 0 & 0 & 0 & -2 & 1 & 0 & 0 & 0 & 0 & 0 & 0 \\ 0 & 0 & 1 & 0 & 0 & 0 & 0 & 0 & 0 & 1 & -2 & 1 & 0 & 0 & 0 & 0 & 0 \\ 0 & 0 & 0 & 1 & 0 & 0 & 0 & 0 & 0 & 0 & 1 & -2 & 1 & 0 & 0 & 0 & 0 \\ 0 & 1 & 0 & 0 & 0 & 0 & 0 & 0 & 0 & 0 & 0 & 1 & -2 & 1 & 0 & 0 & 0 \\ 0 & 0 & 1 & 0 & 0 & 0 & 0 & 0 & 0 & 0 & 0 & 0 & 1 & -2 & 0 & 0 & 0 \\ 0 & 1 & 0 & 0 & 0 & 0 & 0 & 0 & 0 & 0 & 0 & 0 & 0 & 0 & -2 & 1 & 0 \\ 0 & 0 & 1 & 0 & 0 & 0 & 0 & 0 & 0 & 0 & 0 & 0 & 0 & 0 & 1 & -2 & 1 \\ 0 & 0 & 0 & 1 & 0 & 0 & 0 & 0 & 0 & 0 & 0 & 0 & 0 & 0 & 0 & 1 & -2\end{array}\right)$

and let $S=\left\{e_{1}, e_{2}, e_{3}, e_{4}\right\}$. Then the $G(F)$-orbits of an open subset of $V(F)$ are in bijection with the $F$-points of an open subvariety of the moduli space $\mathcal{M}_{\Lambda, S}$ of $K 3$ surfaces $X$ lattice-polarized by $(\Lambda, S)$.

12.1. Néron-Severi lattice and moduli problem. Since quadruply symmetric penteracts are also triply symmetric in, say, the last three coordinates, we may use our constructions from Section 9 to help us analyze these. In particular, the $\mathrm{K} 3$ surface $X_{123}\left(=X_{1 i j}\right.$ for any $\left.2 \leqslant i<j\right)$ has at least six rank singularities (over $\bar{F}$ ), and a numerical example shows that it then generically has exactly six singular points. Meanwhile, the K3 surfaces $X_{i j k}$ for $2 \leqslant i<j<k \leqslant 5$ and all $X_{i j k l}$ are generically nonsingular, and the maps from $X_{1234}$ to $X_{1 i j}$ for $2 \leqslant i<j \leqslant 4$ blow down lines to the six singular points on $X_{1 i j}$. We thus have at least 18 lines on $X_{1234}$. Denote the lines coming from $X_{123}, X_{124}$, and $X_{134}$ by $E_{\ell 4}, E_{\ell 3}$, and $E_{\ell 2}$, respectively, for $1 \leqslant \ell \leqslant 6$.

The map $X_{1234} \rightarrow \mathbb{P}\left(V_{1}^{\vee}\right)$ is a genus one fibration whose discriminant as a binary form on $V_{1}$ is of degree 24 and factors as the sixth power of a degree three form times an irreducible degree six form. An argument similar to the doubly doubly symmetric case shows that this fibration indeed has three reducible fibers 
of type $\mathrm{I}_{6}$, that is, these reducible fibers each consist of six lines in a 'hexagon'. These three sets of six lines, yielding a total of 18 lines, correspond exactly to the 18 lines in the previous paragraph (each set of six lines in the previous paragraph contains one pair of parallel lines from each of the three hexagons).

These lines can be written down very explicitly. Let $r_{i}$ for $1 \leqslant i \leqslant 3$ be the three points in $\mathbb{P}\left(V_{1}^{\vee}\right)$ over which the fibration has reducible fibers. Then the six singular points in $X_{123}$ are of the form $\left(r_{i}, s_{i}, t_{i}\right)$ and $\left(r_{i}, t_{i}, s_{i}\right)$ for $1 \leqslant i \leqslant 3$, and each hexagon in $X_{1234}$ consists of the lines (in cyclic order)

$$
\begin{aligned}
& E_{i, 4}=\left(r_{i}, s_{i}, t_{i}, *\right), \quad E_{i, 3}=\left(r_{i}, s_{i}, *, t_{i}\right), \quad E_{i, 2}=\left(r_{i}, *, s_{i}, t_{i}\right), \\
& E_{i+3,4}=\left(r_{i}, t_{i}, s_{i}, *\right), \quad E_{i+3,3}=\left(r_{i}, t_{i}, *, s_{i}\right), \quad E_{i+3,2}=\left(r_{i}, *, t_{i}, s_{i}\right) .
\end{aligned}
$$

The three projections $X_{1234} \rightarrow \mathbb{P}\left(V_{2}^{\vee}\right)$ are genus one fibrations with six $\mathrm{I}_{3}$ fibers, that is, triangles of lines. Two of the lines in each triangle come from our 18 lines. For example, for the projection to the second factor, the triangles contain the pair $\left(r_{i}, s_{i}, t_{i}, *\right)$ over $s_{i}$ and $\left(r_{i}, s_{i}, *, t_{i}\right)$, or the pair $\left(r_{i}, t_{i}, s_{i}, *\right)$ and $\left(r_{i}, t_{i}, *, s_{i}\right)$ over $t_{i}$.

If $L_{1}, L_{2}, L_{3}$, and $L_{4}$ denote the pullbacks of $\mathcal{O}_{\mathbb{P}\left(V_{i}\right)^{\vee}}(1)$ to $X_{1234}$ via the projection maps, we obtain relations like in Lemma 7.2:

$$
\begin{aligned}
& L_{1}+L_{2}+L_{3}=2 L_{4}+\sum_{\ell=1}^{6} E_{\ell 4}, \\
& L_{1}+L_{2}+L_{4}=2 L_{3}+\sum_{\ell=1}^{6} E_{\ell 3},
\end{aligned}
$$

and

$$
L_{1}+L_{3}+L_{4}=2 L_{2}+\sum_{\ell=1}^{6} E_{\ell 2} .
$$

The nonzero intersections of these divisors are as follows:

$$
\begin{aligned}
L_{i} \cdot L_{j} & =2 \quad \text { if } i \neq j, \\
L_{i} \cdot E_{\ell i} & =1, \\
E_{i, j} \cdot E_{i^{\prime}, j^{\prime}} & =1 \quad \text { if } i=i^{\prime} \text { and }\left|j-j^{\prime}\right|=1, \\
& \quad \text { or } \quad \text { if }\left|i-i^{\prime}\right|=3 \text { and }\left|j-j^{\prime}\right|=2 .
\end{aligned}
$$

Thus, the Néron-Severi lattice of $\left(X_{1234}\right)_{\bar{F}}$ has rank $17=3 \cdot 5+2$ and discriminant 96. A basis for the lattice is given by the divisor classes $L_{1}, L_{2}$, $L_{3}, L_{4}, E_{12}, E_{13}, E_{14}, E_{42}, E_{43}, E_{22}, E_{23}, E_{24}, E_{52}, E_{53}, E_{31}, E_{32}, E_{33}$, and the corresponding Gram matrix is in (30). 
PROPOSITION 12.2. For a very general $X$ in this family of $K 3$ surfaces, $\overline{\mathrm{NS}}(X)$ is spanned by the above divisors.

Proof. We give a proof using elliptic fibrations. Let $e_{1}, \ldots, e_{22}$ be the divisor classes $L_{1}, \ldots, L_{4}, E_{12}, \ldots, E_{62}, E_{13}, \ldots, E_{63}, E_{14}, \ldots, E_{64}$, and $f_{1}, \ldots, f_{17}$ the basis chosen. Consider the elliptic fibration with fiber class $e_{2}=f_{2}$ (that is, projection to $\mathbb{P}\left(V_{2}^{\vee}\right)$ ). A section is given by $Z=2 f_{2}-f_{5}+f_{6}+2 f_{8}+f_{9}$, which we take to be our zero section. Then $\left\{e_{6}, e_{7}\right\},\left\{e_{9}, e_{10}\right\},\left\{e_{12}, e_{13}\right\},\left\{e_{15}, e_{16}\right\}$, $\left\{e_{18}, e_{19}\right\},\left\{e_{20}, e_{21}\right\}$ are the nonidentity components of the six $\mathrm{I}_{3}$ fibers. A 3-torsion section is given by $-f_{1}+f_{2}+f_{4}+F$. The sections $e_{8}, e_{11}, e_{17}$ have Néron-Tate height pairing

$$
\left(\begin{array}{ccc}
\frac{8}{3} & 4 & 4 \\
4 & \frac{20}{3} & 6 \\
4 & 6 & \frac{20}{3}
\end{array}\right)
$$

The determinant of this matrix is $32 / 27$, and therefore the discriminant of the lattice spanned by these sections and the fibers is $(32 / 27) \cdot 3^{6} / 3^{2}=96$. Therefore, it is equal to the lattice $\Lambda$ spanned by $e_{1}, \ldots, e_{22}$.

We now have to show that $\Lambda$ is equal to $\overline{\mathrm{NS}}(X)$ for a very general such $\mathrm{K} 3$ surface. As usual, the ranks agree (because the dimension of the moduli space here is $2 \cdot 5-7=3$ ), so we only need to show $\Lambda$ is saturated. Since the discriminant is $96=2^{5} \cdot 3$, we merely need to show that it is 2 -saturated. This can only fail to happen if there is a 2-torsion section (which is not possible because of the fiber configuration), or if the Mordell-Weil lattice is larger. A direct calculation (by checking all $2^{3}-1$ representatives) shows that a representative of a nonzero class in $\Lambda^{*} / \Lambda$ would have height $m / 3$, for $m$ an odd number. But the height of a section $P$ equals $4+2(P \cdot O)$ minus the sum of the contributions from the fibers, which are 0 or $2 / 3$, and hence is an even number divided by 3 . It follows that $\Lambda$ is saturated.

Proof of Theorem 12.1. The above discussion shows that a quadruply symmetric penteract gives rise to a $\mathrm{K} 3$ surface lattice-polarized by $(\Lambda, S)$. For the reverse, the proof is an obvious generalization of that of Theorem 9.1. The main argument within (coming from the proof of Theorem 8.1) needs to be repeated three times to show that the constructed penteract is symmetric with respect to three transpositions, for example, (25), (35), and (45), of the tensor factors (under identifications of the corresponding vector spaces). 
12.2. Automorphisms. The automorphisms $\alpha_{k l, m}$ and $\Phi_{i j k l m}$ also apply in this case. All of the 5-cycles, by the symmetry, act in equivalent ways, and in particular, $\Phi_{54321}$ induces the action of the matrix

$$
\left(\begin{array}{ccccccccccccccccc}
-1 & 2 & 2 & 0 & 0 & 0 & 0 & 0 & 0 & 0 & 0 & 0 & 0 & 0 & 0 & 0 & 0 \\
-1 & 1 & 1 & 1 & 0 & 0 & 0 & 0 & 0 & 0 & 0 & 0 & 0 & 0 & 0 & 0 & 0 \\
0 & 1 & 0 & 0 & 0 & 0 & 0 & 0 & 0 & 0 & 0 & 0 & 0 & 0 & 0 & 0 & 0 \\
0 & 0 & 1 & 0 & 0 & 0 & 0 & 0 & 0 & 0 & 0 & 0 & 0 & 0 & 0 & 0 & 0 \\
1 & 0 & 0 & 0 & -1 & -1 & -1 & -1 & -1 & 0 & 0 & 0 & 0 & 0 & 0 & 0 & 0 \\
-1 & 0 & 1 & 0 & 0 & 1 & 1 & 1 & 1 & 0 & 0 & 0 & 0 & 0 & 0 & 0 & 0 \\
0 & 1 & 0 & 0 & 0 & -1 & -1 & 0 & 0 & 0 & 0 & 0 & 0 & 0 & 0 & 0 & 0 \\
0 & 0 & 0 & 0 & 0 & 0 & 1 & 0 & 0 & 0 & 0 & 0 & 0 & 0 & 0 & 0 & 0 \\
0 & 0 & 1 & 0 & 0 & 0 & -1 & -1 & 0 & 0 & 0 & 0 & 0 & 0 & 0 & 0 & 0 \\
1 & 0 & 0 & 0 & 0 & 0 & 0 & 0 & 0 & -1 & -1 & -1 & -1 & -1 & 0 & 0 & 0 \\
-1 & 0 & 1 & 0 & 0 & 0 & 0 & 0 & 0 & 0 & 1 & 1 & 1 & 1 & 0 & 0 & 0 \\
0 & 1 & 0 & 0 & 0 & 0 & 0 & 0 & 0 & 0 & -1 & -1 & 0 & 0 & 0 & 0 & 0 \\
0 & 0 & 0 & 0 & 0 & 0 & 0 & 0 & 0 & 0 & 0 & 1 & 0 & 0 & 0 & 0 & 0 \\
0 & 0 & 1 & 0 & 0 & 0 & 0 & 0 & 0 & 0 & 0 & -1 & -1 & 0 & 0 & 0 & 0 \\
-1 & 1 & 1 & -2 & 1 & 1 & 0 & 1 & 1 & 1 & 1 & 0 & 1 & 1 & 0 & 0 & -1 \\
1 & -1 & 0 & 2 & -1 & -1 & 0 & -1 & -1 & -1 & -1 & 0 & -1 & -1 & -1 & 0 & 1 \\
0 & 1 & 0 & 0 & 0 & 0 & 0 & 0 & 0 & 0 & 0 & 0 & 0 & 0 & 0 & -1 & -1
\end{array}\right)
$$

on the divisors $\left\{L_{1}, L_{2}, L_{3}, L_{4}, E_{12}, \ldots, E_{62}, E_{13}, \ldots, E_{63}, E_{14}, \ldots, E_{64}\right\}$ in $\overline{\mathrm{NS}}(X)$. This automorphism has order 4 and is fixed-point-free; it will be discussed further in Section 17.3. Note, however, that the square of $\Phi_{54321}$ is an involution but not fixed-point-free over $\bar{F}$ (see Section 13.2 for a detailed explanation, which also applies to this case).

\section{Quintuply symmetric penteracts: $\operatorname{Sym}^{5}(2)$}

Let us now consider a quintuply symmetric penteract. We will prove that the general orbits of such tensors correspond to certain K3 surfaces with Picard rank at least 18 over $\bar{F}$. Note that such penteracts also have an interpretation as binary quintic forms, that is, degree 5 subschemes of the projective line.

THEOREM 13.1. Let $V=\operatorname{Sym}^{5} V_{1}$ for a 2-dimensional $F$-vector space $V_{1}$. Let $G^{\prime}$ be the group $\mathbb{G}_{m} \times \mathrm{GL}\left(V_{1}\right)$ and let $G$ be the quotient of $G^{\prime}$ by the kernel of the multiplication map $\mathbb{G}_{m} \times \mathbb{G}_{m} \rightarrow \mathbb{G}_{m}$ given by $\left(\gamma_{1}, \gamma_{2}\right) \mapsto \gamma_{1} \gamma_{2}^{5}$. Let $\Lambda$ be the lattice whose Gram matrix is 


$$
\left(\begin{array}{cccccccccccccccccc}
-2 & 0 & 0 & 0 & 0 & 0 & 1 & 0 & 0 & 0 & 0 & 0 & 0 & 0 & 0 & 0 & 0 & 1 \\
0 & -2 & 0 & 0 & 0 & 0 & 0 & 1 & 0 & 0 & 0 & 1 & 0 & 0 & 0 & 0 & 0 & 0 \\
0 & 0 & -2 & 0 & 0 & 0 & 0 & 0 & 1 & 0 & 0 & 0 & 1 & 0 & 0 & 0 & 0 & 0 \\
0 & 0 & 0 & -2 & 0 & 0 & 0 & 0 & 0 & 1 & 0 & 0 & 0 & 0 & 0 & 1 & 0 & 0 \\
0 & 0 & 0 & 0 & -2 & 0 & 0 & 0 & 0 & 0 & 1 & 0 & 0 & 0 & 1 & 0 & 1 & 0 \\
0 & 0 & 0 & 0 & 0 & -2 & 0 & 0 & 0 & 0 & 0 & 1 & 0 & 0 & 0 & 0 & 1 & 0 \\
1 & 0 & 0 & 0 & 0 & 0 & -2 & 0 & 0 & 0 & 0 & 0 & 1 & 0 & 0 & 1 & 0 & 0 \\
0 & 1 & 0 & 0 & 0 & 0 & 0 & -2 & 0 & 0 & 0 & 0 & 0 & 1 & 0 & 0 & 0 & 0 \\
0 & 0 & 1 & 0 & 0 & 0 & 0 & 0 & -2 & 0 & 0 & 0 & 0 & 0 & 0 & 0 & 0 & 0 \\
0 & 0 & 0 & 1 & 0 & 0 & 0 & 0 & 0 & -2 & 0 & 0 & 0 & 0 & 1 & 0 & 0 & 1 \\
0 & 0 & 0 & 0 & 1 & 0 & 0 & 0 & 0 & 0 & -2 & 0 & 0 & 0 & 0 & 0 & 0 & 0 \\
0 & 1 & 0 & 0 & 0 & 1 & 0 & 0 & 0 & 0 & 0 & -2 & 0 & 0 & 0 & 1 & 0 & 0 \\
0 & 0 & 1 & 0 & 0 & 0 & 1 & 0 & 0 & 0 & 0 & 0 & -2 & 0 & 0 & 0 & 1 & 0 \\
0 & 0 & 0 & 0 & 0 & 0 & 0 & 1 & 0 & 0 & 0 & 0 & 0 & -2 & 0 & 0 & 0 & 1 \\
0 & 0 & 0 & 0 & 1 & 0 & 0 & 0 & 0 & 1 & 0 & 0 & 0 & 0 & -2 & 0 & 0 & 0 \\
0 & 0 & 0 & 1 & 0 & 0 & 1 & 0 & 0 & 0 & 0 & 1 & 0 & 0 & 0 & -2 & 0 & 0 \\
0 & 0 & 0 & 0 & 1 & 1 & 0 & 0 & 0 & 0 & 0 & 0 & 1 & 0 & 0 & 0 & -2 & 0 \\
1 & 0 & 0 & 0 & 0 & 0 & 0 & 0 & 0 & 1 & 0 & 0 & 0 & 1 & 0 & 0 & 0 & -2
\end{array}\right)
$$

and let $S=\left\{e_{1}, e_{2}, e_{3}, e_{4}\right\}$. Then the $G(F)$-orbits of an open subset of $V(F)$ are in bijection with the $F$-points of an open subvariety of the moduli space $\mathcal{M}_{\Lambda, S}$ of $K 3$ surfaces $X$ lattice-polarized by $(\Lambda, S)$.

13.1. Néron-Severi lattice and moduli problem. As before, we may use the constructions from previous sections; in particular, a quintuply symmetric penteract is also quadruply symmetric in any four coordinates. By the results of Section 12, all the K3 surfaces $X_{i j k}$ (for any $1 \leqslant i<j<k \leqslant 5$ ) has at least six rank singularities (over $\bar{F}$ ), and it is easy to check numerically that $X_{i j k}$ generically has exactly six singular points. Meanwhile, the K3 surfaces $X_{i j k l}$ for $1 \leqslant i<j<k<l \leqslant 5$ are generically nonsingular, and for $1 \leqslant i<j<k \leqslant 4$, the projections $X_{1234} \rightarrow X_{i j k}$ blow up the six singular points lying on each of the four surfaces $X_{i j k}$, yielding 24 lines on $X_{1234}$.

For $\{i, j, k, m\}=\{1,2,3,4\}$, we denote the six lines coming from the blowup $X_{1234} \rightarrow X_{i j k}$ by $E_{\sigma}$, for the permutations $\sigma$ in the symmetric group $S_{4}$ with $\sigma^{-1}(1)=m$. Let $L_{i}$ denote the pullback of the line bundle $\mathcal{O}_{\mathbb{P}\left(V_{i}^{\vee}\right)}(1)$ to $X_{1234}$ via the projection. By Lemma 7.2, we have

$$
L_{i}+L_{j}+L_{k}=2 L_{m}+\sum_{\substack{\sigma \in S_{4} \\ \sigma^{-1}(1)=m}} E_{\sigma}
$$




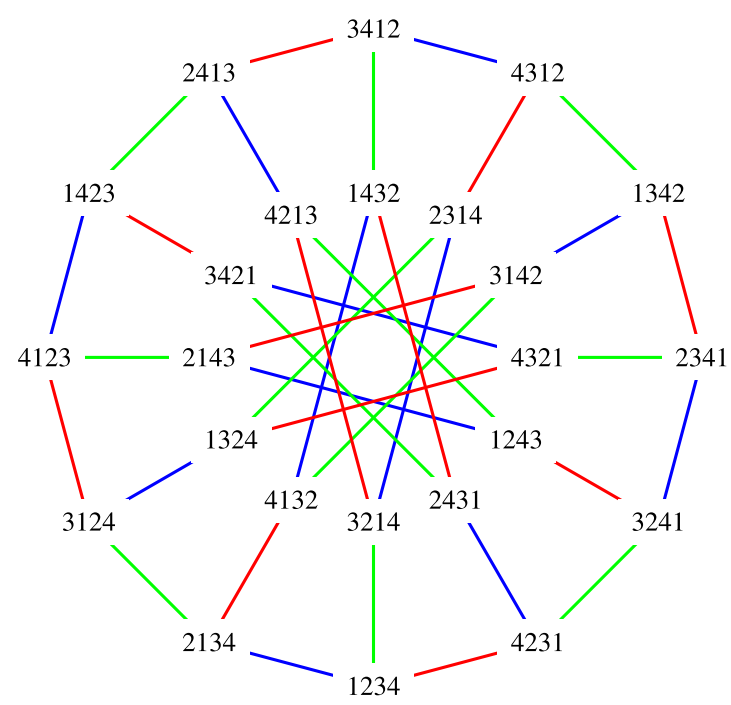

Figure 3. The intersection graph as a Cayley graph of $S_{4}$ (also known as the Nauru graph [33]). Each vertex is given by an element $\sigma \in S_{4}$ (represented by the string $\sigma(1) \ldots \sigma(4))$, and the blue, green, and red edges correspond to the actions of the transposition (12), (13), or (14), respectively.

Because of the symmetry, if $(a, b, c)$ is one of the singular points in $X_{123}$, then the other five singular points are just permutations of the three coordinates. Therefore, the 6 lines $E_{\sigma}$ in $X_{1234}$ obtained from blowing up the six singular points in $X_{123}$ are given by $\{(\tau(a), \tau(b), \tau(c), *)\} \subset X_{1234}$, for each permutation $\tau \in S_{3}$, where $*$ means that any point of $\mathbb{P}^{1}$ may be used. More generally, the 24 lines are given by the permutations of $(a, b, c, *)$. Each line intersects exactly one of the lines in the other three sets of 6 , namely when two of their non-* coordinates coincide. If we view these 24 lines as vertices of a graph, with the edges corresponding to the 36 intersection points, this graph is the generalized Petersen graph on 12 vertices.

To relate the graph in Figure 3 to the lines in our K3 surface $X_{1234}$, note that the vertex corresponding to $\sigma \in S_{4}$ represents the line given by the action of $\sigma$ on the ordered set $(*, a, b, c)$. For example, the bottom vertex is the line $(*, a, b, c)$ and intersects the lines $(a, *, b, c),(b, a, *, c)$, and $(c, a, b, *)$.

Each of the projections $\pi_{i}: X_{1234} \rightarrow \mathbb{P}\left(V_{1}^{\vee}\right)$ to the $i$ th factor, for $1 \leqslant i \leqslant 4$, is a genus one fibration whose discriminant as a binary form on $V_{1}$ is again of degree 24 and factors as the sixth power of a degree three form times an irreducible degree six form. An argument similar to the quadruply symmetric case shows that 
this fibration indeed has three reducible fibers of type $\mathrm{I}_{6}$, that is, these reducible fibers each consist of six lines in a 'hexagon'. These account for 18 of the 24 lines encountered earlier, namely the $E_{\sigma}$ for $\sigma^{-1}(1) \neq i$; the other 6 lines, via this projection $\pi_{i}$, in fact cover the entire $\mathbb{P}\left(V_{1}^{\vee}\right)$. Thus, the intersection of the 24 lines with one of the reducible fibers is exactly a hexagon of lines and 6 distinct points.

The intersections among all these divisors can be described as follows:

$$
\begin{aligned}
L_{i} \cdot L_{j} & =2\left(1-\delta_{i j}\right), \quad L_{i} \cdot E_{\sigma}=\delta_{i, \sigma^{-1}(1)}, \quad E_{\sigma}^{2}=-2, \\
E_{\sigma} \cdot E_{\sigma^{\prime}} & = \begin{cases}1 & \text { if there is an edge between the corresponding vertices } \\
0 & \text { otherwise }\end{cases}
\end{aligned}
$$

The intersection matrix has rank 18 , and the lattice generated by these divisors has discriminant 20, so the Néron-Severi lattice of $\left(X_{1234}\right)_{\bar{F}}$ has Gram matrix (31) and has rank 18. A basis for the lattice is given by $L_{1}, L_{2}, L_{3}, L_{4}, E_{4321}, E_{4312}$, $E_{4231}, E_{4132}, E_{4213}, E_{3421}, E_{3412}, E_{2431}, E_{1432}, E_{2413}, E_{3241}, E_{3142}, E_{3214}$ and $E_{2134}$.

Proposition 13.2. Let $\Lambda$ be the lattice spanned by the classes of the above divisors $E_{\sigma}$. Then for the $K 3$ surface $X$ arising from a very general quintuply symmetric penteract, we have $\overline{\mathrm{NS}}(X)=\Lambda$.

Proof. First, note that the dimension of the moduli space of quintuply symmetric penteracts is $6-4=2$, so the rank of $\overline{\mathrm{NS}}(X)$ for a very general $X$ is 18 , which is the rank of $\Lambda$.

Consider the elliptic fibration $X \rightarrow \mathbb{P}\left(V_{1}^{\vee}\right)$. Generically, the root lattice formed by the nonidentity components of the reducible fibers is $A_{5}^{3}$. Since $E_{(1)}=E_{1234}$ intersects the fiber class in 1, it follows that the elliptic fibration has a section. The root sublattice has rank 15 and discriminant $6^{3}$. We check that the (Jacobian of) the elliptic fibration has a 3-torsion section, in fact defined over the ground field, and since the Picard number is 18 , the only possibility is to have a nontorsion section of height $20 /(216 / 9)=5 / 6=4-5 / 6-5 / 6-9 / 6$. We can also check directly that there are no 2-torsion sections, even over the algebraic closure. For the Picard group to be any larger, it would have to have discriminant 5, and a Mordell-Weil generator of height $5 / 24$, which is impossible with the fiber configuration.

Proof of Theorem 13.1. The above discussion shows that a quintuply symmetric penteract produces a K3 surface that is lattice-polarized by $(\Lambda, S)$. For the other direction, like for Theorem 12.1, the proof is a straightforward generalization of the proof of Theorem 9.1. We construct a penteract from this data, and applying the argument from Theorem 8.1 four times shows that all five vector spaces related to the penteract may be identified and that the penteract is symmetric with respect to any two. 
13.2. Automorphisms. Given a $\mathrm{K} 3$ surface $X$ coming from a quintuply symmetric penteract, the visible automorphisms of $X$ may be described quite easily.

Because of the symmetry in this case, all the automorphisms of the form $\alpha_{k l, m}$ on $X_{i j k l}$ act in similar ways. As always, each $\alpha_{k l, m}$ is an involution of the K3 surface $X_{i j k l}$ switching the $k$ th and $l$ th coordinates, for example, $\alpha_{34,5}$ sends ( $a$, $b, c, d) \in X_{1234}$ to $(a, b, d, c)$. Thus, these generate a group of automorphisms isomorphic to $S_{4}$. Note that the automorphism $\Phi_{i j k l m}$ introduced in Section 7.4 is an order 4 element, as the composition of three of these transpositions.

While $\Phi_{i j k l m}$ is fixed-point-free for the general $X$ in this family (see Section 17.2 or simply observe that the diagonal $\mathbb{P}^{1}$ in $\left(\mathbb{P}^{1}\right)^{4}$ does not generally intersect $X$ ), note that its square is an involution but not fixed-point-free (over $\bar{F})$. For example, the $\mathbb{P}^{1} \times \mathbb{P}^{1}$ of points $(a, b, a, b) \in\left(\mathbb{P}^{1}\right)^{4}$ on $X_{1234}$ will be fixed under $\Phi_{i j k l m}^{2}$; in particular, for the general $X$ in this family, there will be 8 fixed points over $\bar{F}$, namely the intersection of $X$ with this diagonal $\mathbb{P}^{1} \times \mathbb{P}^{1}$ in $\left(\mathbb{P}^{1} \times \mathbb{P}^{1}\right) \times\left(\mathbb{P}^{1} \times \mathbb{P}^{1}\right)$.

\section{4. $2 \otimes 2 \otimes 2 \otimes 4$}

In this section, we study the space of $2 \times 2 \times 2 \times 4$ matrices and classify their orbits in terms of certain K3 surfaces of rank at least 13 over $\bar{F}$ :

THEOREM 14.1. Let $V=V_{1} \otimes V_{2} \otimes V_{3} \otimes U$, where $V_{1}, V_{2}$, and $V_{3}$ are 2dimensional $F$-vector spaces and $U$ is a 4-dimensional $F$-vector space. Let $G^{\prime}=\mathrm{GL}\left(V_{1}\right) \times \mathrm{GL}\left(V_{2}\right) \times \mathrm{GL}\left(V_{3}\right) \times \mathrm{GL}(U)$, and let $G$ be the quotient of $G^{\prime}$ by the kernel of the multiplication map $\mathbb{G}_{m}^{4} \rightarrow \mathbb{G}_{m}$. Let $\Lambda$ be the lattice whose Gram matrix is

$$
\left(\begin{array}{ccccccccccccc}
4 & 4 & 4 & 4 & 0 & 0 & 0 & 0 & 0 & 0 & 0 & 0 & 0 \\
4 & 0 & 2 & 2 & 0 & 0 & 0 & 1 & 1 & 1 & 1 & 1 & 1 \\
4 & 2 & 0 & 2 & 1 & 1 & 1 & 0 & 0 & 0 & 1 & 1 & 1 \\
4 & 2 & 2 & 0 & 1 & 1 & 1 & 1 & 1 & 1 & 0 & 0 & 0 \\
0 & 0 & 1 & 1 & -2 & 0 & 0 & 0 & 0 & 0 & 0 & 0 & 0 \\
0 & 0 & 1 & 1 & 0 & -2 & 0 & 0 & 0 & 0 & 0 & 0 & 0 \\
0 & 0 & 1 & 1 & 0 & 0 & -2 & 0 & 0 & 0 & 0 & 0 & 0 \\
0 & 1 & 0 & 1 & 0 & 0 & 0 & -2 & 0 & 0 & 0 & 0 & 0 \\
0 & 1 & 0 & 1 & 0 & 0 & 0 & 0 & -2 & 0 & 0 & 0 & 0 \\
0 & 1 & 0 & 1 & 0 & 0 & 0 & 0 & 0 & -2 & 0 & 0 & 0 \\
0 & 1 & 1 & 0 & 0 & 0 & 0 & 0 & 0 & 0 & -2 & 0 & 0 \\
0 & 1 & 1 & 0 & 0 & 0 & 0 & 0 & 0 & 0 & 0 & -2 & 0 \\
0 & 1 & 1 & 0 & 0 & 0 & 0 & 0 & 0 & 0 & 0 & 0 & -2
\end{array}\right),
$$


and let $S=\left\{e_{1}, e_{2}, e_{3}, e_{4}\right\}$. Then the $G(F)$-orbits of an open subset of $V(F)$ are in bijection with the $F$-rational points of an open subvariety of the moduli space $\mathcal{M}_{\Lambda, S}$ of $K 3$ surfaces $X$ lattice-polarized by $(\Lambda, S)$.

14.1. Construction of $\mathbf{K} 3$ surfaces. Given an element $A \in V(K)$, we construct a K3 surface $X$ with Picard number at least 13 as follows. We will show that the intersection of the varieties defined by the equations

$$
\begin{aligned}
& A\left(v_{1}, v_{2}, \cdot, u\right) \equiv 0 \\
& A\left(v_{1}, \cdot, v_{3}, u\right) \equiv 0 \\
& A\left(\cdot, v_{2}, v_{3}, u\right) \equiv 0
\end{aligned}
$$

in $\mathbb{P}\left(V_{1}^{\vee}\right) \times \mathbb{P}\left(V_{2}^{\vee}\right) \times \mathbb{P}\left(V_{3}^{\vee}\right) \times \mathbb{P}\left(U^{\vee}\right)$ is a K3 surface $X$. The projection $X_{U}$ of $X$ to $\mathbb{P}\left(U^{\vee}\right)$ is then a quartic surface with 12 singularities over $\bar{F}$ (to be described below).

The projection $X_{123}$ of $X$ to $\mathbb{P}\left(V_{1}^{\vee}\right) \times \mathbb{P}\left(V_{2}^{\vee}\right) \times \mathbb{P}\left(V_{3}^{\vee}\right)$ is cut out by a single tridegree $(2,2,2)$ form $f\left(v_{1}, v_{2}, v_{3}\right)$. In order to explicitly describe this form, let us write $A$ as a quadruple $\left(A_{1}, A_{2}, A_{3}, A_{4}\right)$ of trilinear forms on $V_{1}^{\vee} \times V_{2}^{\vee} \times V_{3}^{\vee}$ (by choosing a basis for $U$ ), and consider the determinant

$$
\begin{aligned}
& D\left(v_{1}, v_{1}^{\prime}, v_{2}, v_{2}^{\prime}, v_{3}, v_{3}^{\prime}\right)
\end{aligned}
$$

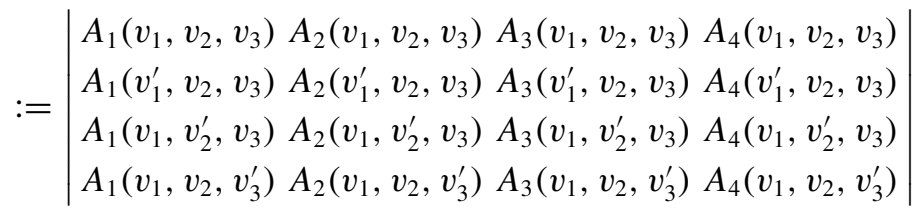

for vectors $v_{1}, v_{1}^{\prime} \in V_{1}^{\vee}, v_{2}, v_{2}^{\prime} \in V_{2}^{\vee}, v_{3}, v_{3}^{\prime} \in V_{3}^{\vee}$. Then we observe that if $\left(v_{1}\right.$, $\left.v_{2}, v_{3}, v_{4}\right) \in V_{1}^{\vee} \times V_{2}^{\vee} \times V_{3}^{\vee} \times U$ satisfies equations (34)-(36), then $u \in U^{\vee}$ lies in the (right) kernel of the matrix in (37). Furthermore, since the determinant $D$ vanishes if $v_{1}=c v_{1}^{\prime}, v_{2}=c v_{2}^{\prime}$, or $v_{3}=c v_{3}^{\prime}$ for any constant $c \in K$, we see that the polynomial $D\left(v_{1}, v_{1}^{\prime}, v_{2}, v_{2}^{\prime}, v_{3}, v_{3}^{\prime}\right)$ is a multiple of $\operatorname{det}\left(v_{1}, v_{1}^{\prime}\right) \operatorname{det}\left(v_{2}, v_{2}^{\prime}\right) \operatorname{det}\left(v_{3}\right.$, $\left.v_{3}^{\prime}\right)$. The tridegree $(2,2,2)$ form

$$
f\left(v_{1}, v_{2}, v_{3}\right):=\frac{D\left(v_{1}, v_{1}^{\prime}, v_{2}, v_{2}^{\prime}, v_{3}, v_{3}^{\prime}\right)}{\operatorname{det}\left(v_{1}, v_{1}^{\prime}\right) \operatorname{det}\left(v_{2}, v_{2}^{\prime}\right) \operatorname{det}\left(v_{3}, v_{3}^{\prime}\right)}
$$

is then easily checked to be irreducible and thus defines the projection $X_{123}$ of $X$ onto $\mathbb{P}\left(V_{1}^{\vee}\right) \times \mathbb{P}\left(V_{2}^{\vee}\right) \times \mathbb{P}\left(V_{3}^{\vee}\right)$.

One checks that generically $X_{123}$ is smooth, and thus $X$ and $X_{123}$ are isomorphic. Moreover, for $\{i, j, k\}=\{1,2,3\}$, we have that $X_{123}$ is a double cover of 
$\mathbb{P}\left(V_{i}^{\vee}\right) \times \mathbb{P}\left(V_{j}\right)^{\vee}$ branched over a genus 9 curve, given by a bidegree $(4,4)$ equation (namely, the discriminant of $f$ viewed as a quadratic form on $\mathbb{P}\left(V_{k}^{\vee}\right)$ ).

Let $X_{U}$ be the image of $X$ under the fourth projection to $\mathbb{P}\left(U^{\vee}\right)$. Then $u \in X_{U}$ if and only if there exists $\left(v_{1}, v_{2}, v_{3}\right) \in \mathbb{P}\left(V_{1}^{\vee}\right) \times \mathbb{P}\left(V_{2}^{\vee}\right) \times \mathbb{P}\left(V_{3}^{\vee}\right)$ such that the equations (34)-(36) are satisfied, which occurs if and only if the $2 \times 2 \times 2$ cube $A(\cdot, \cdot, \cdot, u)=A(u)$ has discriminant 0 . (Recall that $2 \times 2 \times 2$ cubes have a single $\mathrm{SL}_{2} \times \mathrm{SL}_{2} \times \mathrm{SL}_{2}$-invariant of degree four called the discriminant, which is the discriminant of each of the three binary quadratics that arise from the determinant construction on the cube. If this discriminant vanishes, the cube is called singular, and in this case, all three of the binary quadratics are multiples of squares of linear forms, that is, have double roots in $\mathbb{P}^{1}$.) We conclude that $X_{U}$ is given by the vanishing of the quartic polynomial disc $A(u)$.

We may also give the following alternative description of $X_{U}$. Let $Y_{12}, Y_{13}$, and $Y_{23}$ denote the threefolds in $\mathbb{P}\left(V_{1}^{\vee}\right) \times \mathbb{P}\left(V_{2}^{\vee}\right) \times \mathbb{P}\left(U^{\vee}\right), \mathbb{P}\left(V_{1}^{\vee}\right) \times \mathbb{P}\left(V_{3}^{\vee}\right) \times \mathbb{P}\left(U^{\vee}\right)$, and $\mathbb{P}\left(V_{2}^{\vee}\right) \times \mathbb{P}\left(V_{3}^{\vee}\right) \times \mathbb{P}\left(U^{\vee}\right)$ defined by (34), (35), and (36), respectively. Then for $(i, j) \in\{(1,2),(1,3),(2,3)\}$, we have that $X_{U}$ is the ramification locus of the double cover $Y_{i j} \rightarrow \mathbb{P}\left(U^{\vee}\right)$ given by projection. To see this, fix $u \in U^{\vee}$; then $A\left(v_{1}\right.$, $\left.v_{2}, \cdot, u\right)=0$ has generically two solutions $\left(v_{1}, v_{2}\right) \in \mathbb{P}\left(V_{1}^{\vee}\right) \times \mathbb{P}\left(V_{2}^{\vee}\right)$, for there are generically two choices for $v_{1}$ as the root of the associated binary quadratic on $V_{1}^{\vee}$, and then a uniquely determined choice for $v_{2}$ given $v_{1}$ (namely, $v_{2}$ is the left kernel of the bilinear form $A\left(v_{1}, \cdot, \cdot, u\right)$ ). If this binary quadratic form on $V_{1}^{\vee}$ has only one root (which occurs when disc $A(u)=0$ ), then there will thus be only one $\left(v_{1}, v_{2}\right)$ giving $A\left(v_{1}, v_{2}, \cdot, u\right)$. Hence $X_{U}$ is the ramification locus of the double cover $Y_{12} \rightarrow \mathbb{P}\left(U^{\vee}\right)$, and similarly is the ramification locus of the double covers $Y_{13} \rightarrow \mathbb{P}\left(U^{\vee}\right)$ and $Y_{23} \rightarrow \mathbb{P}\left(U^{\vee}\right)$. It follows, in particular, that the preimage $X_{i j U}$ of $X_{U}$ in $Y_{i j}$ is the projection of $X$ onto $\mathbb{P}\left(V_{i}^{\vee}\right) \times \mathbb{P}\left(V_{j}^{\vee}\right) \times \mathbb{P}\left(U^{\vee}\right)$ and is isomorphic to $X_{U}$.

14.2. Singularities and exceptional divisors. We claim that $X_{U}$ generically has 12 singularities; these are closely related to certain special sets $S_{i}$ of four points in each $\mathbb{P}\left(V_{i}^{\vee}\right)$. To construct this set $S_{1}$ of four points in $\mathbb{P}\left(V_{1}^{\vee}\right)$, we consider the $2 \times 2 \times 4$ box $A\left(v_{1}, \cdot, \cdot, \cdot\right)=A\left(v_{1}\right)$ attached to a given point $v_{1} \in \mathbb{P}\left(V_{1}^{\vee}\right)$. It has a natural $\operatorname{SL}\left(V_{2}\right) \times \operatorname{SL}\left(V_{3}\right) \times \operatorname{SL}(U)$-invariant-in fact, an $\operatorname{SL}\left(V_{2} \otimes V_{3}\right) \times$ $\mathrm{SL}(U)$-invariant - of degree four, namely the determinant of $A\left(v_{1}\right)$ when viewed as an element of $\left(V_{2} \otimes V_{3}\right) \otimes U$. That is, with a choice of basis, this is simply the determinant of $A\left(v_{1}\right)$ viewed as a $4 \times 4$ matrix. This invariant gives a degree 4 form on $\mathbb{P}\left(V_{1}^{\vee}\right)$, which then cuts out our set $S_{1}$ of four points in $\mathbb{P}\left(V_{1}^{\vee}\right)$ over $\bar{F}$. The sets $S_{i}$ for $i=2,3$ are constructed in the analogous manner.

The sets $S_{i}$ have a further significance. Consider the projection $\pi_{1}: X_{123} \rightarrow$ $\mathbb{P}\left(V_{1}^{\vee}\right)$. The fiber over any point $v_{1} \in V_{1}^{\vee}$ is then the curve in $\mathbb{P}^{1}\left(V_{2}^{\vee}\right) \times \mathbb{P}^{1}\left(V_{3}^{\vee}\right)$ 
defined by the bidegree $(2,2)$-form $f\left(v_{1}, \cdot, \cdot\right)$. We thus see that $X_{123} \cong X$ is a genus one fibration over $\mathbb{P}\left(V_{1}^{\vee}\right)$, where each genus one fiber is described as a bidegree $(2,2)$ curve in $\mathbb{P}^{1}\left(V_{2}^{\vee}\right) \times \mathbb{P}^{1}\left(V_{3}^{\vee}\right)$. A fiber in this fibration is singular precisely when the discriminant of this bidegree $(2,2)$ form, which is a binary form of degree 24 on $\mathbb{P}^{1}\left(V_{1}^{\vee}\right)$, is zero. Using indeterminate entries for $A$, one checks that this degree 24 binary form factors as the square of a binary quartic form times an irreducible binary form of degree 16. Thus, generically, we have 16 nodal fibers, while the remaining four fibers turn out to be banana curves, that is, they have as components two rational curves intersecting in two points, as we now show.

In fact, we claim that $S_{1}$ gives precisely the set of four points over which the fibers for the map $\pi_{1}: X \rightarrow \mathbb{P}\left(V_{1}^{\vee}\right)$ are banana curves. Indeed, for $v_{1} \in S_{1}$, by construction there exists a (generically unique) point $u \in \mathbb{P}\left(U^{\vee}\right)$ such that $A\left(v_{1}\right.$, $\cdot, \cdot, u) \equiv 0$. Then the points $\left(v_{2}, v_{3}\right) \in \mathbb{P}\left(V_{2}^{\vee}\right) \times \mathbb{P}\left(V_{3}^{\vee}\right)$ such that $\left(v_{1}, v_{2}, v_{3}\right.$, $u) \in X$ are cut out by the single equation $A\left(\cdot, v_{2}, v_{3}, u\right) \equiv 0$, which is a bidegree $(1,1)$-form on $\mathbb{P}\left(V_{2}^{\vee}\right) \times \mathbb{P}\left(V_{3}^{\vee}\right)$. It follows that the bidegree $(2,2)$-form that defines the fiber of $\pi_{1}$ over $v_{1}$ factors into two $(1,1)$ forms, as claimed.

Let $E_{j}$ for $1 \leqslant j \leqslant 12$ denote these twelve rational $(1,1)$ curves on $X$ as constructed in the previous paragraph $(1 \leqslant j \leqslant 4$ for $i=1,5 \leqslant j \leqslant 8$ for $i=2$, and $9 \leqslant j \leqslant 12$ for $i=3$ ). Recall that each of these $E_{j}$ must intersect the other rational curve in its fiber in two points. To obtain the other component, we note that for $r \in S_{1}$, the space of $2 \times 2$ matrices spanned by $A_{i}(r, \cdot, \cdot), i=1, \ldots, 4$ is (generically) 3-dimensional, by the definition of $S_{1}$. So there is a plane conic which describes the linear combinations which are of rank 1 . With choices of bases for the vector spaces $V_{i}$ and their duals, suppose such a rank-1 matrix is $Z_{s, t}=\left(\begin{array}{cc}s_{2} t_{2} & -s_{2} t_{1} \\ -s_{1} t_{2} & s_{1} t_{1}\end{array}\right)$; then $\left(s_{1}, s_{2}\right) \in V_{2}^{\vee}$ and $\left(t_{1}, t_{2}\right) \in V_{3}^{\vee}$ give a point $(r, s, t)$ on the fiber over $r$ (the $U$ component may be computed uniquely, and is the linear combination above). The locus of these $(s, t) \in \mathbb{P}\left(V_{2}^{\vee}\right) \times \mathbb{P}\left(V_{3}^{\vee}\right)$ is given by a determinantal condition which says that $Z_{s, t}$ is linearly dependent with the $A_{i}$, hence a $(1,1)$-form.

As we have already noted, the projection $X \rightarrow X_{123}$ is an isomorphism. The map $X \rightarrow X_{U}$ is, not, however: the 12 rational curves $E_{j}$ are blown down to 12 singularities (recall that, for each $j$, the elements of $E_{j}$ all have the same $U$ coordinate $u$ ). Meanwhile, the other rational curves map to nodal curves.

14.3. Néron-Severi lattice. We thus have a number of divisors on $X_{\bar{F}}$ : the $E_{j}$ for $j=1, \ldots, 12$, as well as $H$ (the pullback of $\left.\mathcal{O}_{\mathbb{P}\left(U^{\vee}\right)}(1)\right)$ and the $L_{i}$ (the pullbacks of $\left.\mathcal{O}_{\mathbb{P}\left(V_{i}^{\vee}\right)}(1)\right)$ for $i=1,2,3$. We now compute their intersection numbers.

First, note that $L_{1} \cdot L_{2}=2$ since $X \hookrightarrow \mathbb{P}\left(V_{1}^{\vee}\right) \times \mathbb{P}\left(V_{2}^{\vee}\right) \times \mathbb{P}\left(V_{3}^{\vee}\right)$ is cut out by a $(2$, 2, 2)-form. Intersecting $X$ with the zero loci of $(1,0,0)$ and $(0,1,0)$ forms, we get 
an intersection number of 2 , corresponding to the fact that $X \rightarrow \mathbb{P}\left(V_{1}^{\vee}\right) \times \mathbb{P}\left(V_{2}^{\vee}\right)$ is a double cover. By symmetry, $L_{i} \cdot L_{j}=2$ for all $i \neq j$.

Next, we show that $L_{i} \cdot H=4$. The geometric meaning of the intersection number $L_{3} \cdot H$ is as follows. We fix a point $\left(r_{1}, r_{2}\right) \in \mathbb{P}\left(V_{3}^{\vee}\right)$, that is take a fixed (generic) linear combination of the front and back faces of our $2 \times 2 \times 2$ cube of linear forms, yielding a $2 \times 2$ matrix of linear forms. Intersection with $H$ means that we restrict the forms to a generic hyperplane in $\mathbb{P}\left(U^{\vee}\right)$. We look for the number of points in this plane for which the matrix is singular, and such that $\left(r_{1}, r_{2}\right)$ is the unique linear combination of the faces which is singular. For simplicity, assume that $(1,0)$ is not one of the four special points in $\mathbb{P}\left(V_{3}^{\vee}\right)$ over which $X$ has a reducible fiber. Then, due to the $\operatorname{GL}\left(V_{3}\right)$ action, we may compute the intersection number when $\left(r_{1}, r_{2}\right)$ is $(1,0)$. The constraint that the front face

$$
\left(\begin{array}{ll}
a & b \\
c & d
\end{array}\right)
$$

of our cube is singular describes a conic in the plane $V(H) \subset \mathbb{P}\left(U^{\vee}\right)$. On the other hand, for $(1,0)$ to be the unique linear combination of the faces which makes the matrix singular, if

$$
\left(\begin{array}{ll}
e & f \\
g & h
\end{array}\right)
$$

denotes the back face of our cube, then we also need the mixed determinant

$$
a h+e d-f c-b g
$$

to vanish, and this also describes a plane conic. Generically, these two conics intersect in four points, proving our assertion.

The nonzero intersections among the divisors $H, L_{1}, \ldots, L_{3}, E_{1}, \ldots, E_{12}$ are given by

$$
\begin{aligned}
H^{2}=H \cdot L_{i}=4, & H \cdot E_{i}=0, \\
L_{1} \cdot E_{i}=1 & \text { for } i \in\{5, \ldots, 12\}, \\
L_{2} \cdot E_{i}=1 & \text { for } i \in\{1,2,3,4,9,10,11,12\}, \\
L_{3} \cdot E_{i}=1 & \text { for } i \in\{1, \ldots, 8\} .
\end{aligned}
$$

Next, we determine the Néron-Severi group of the generic K3 surface in this family.

Proposition 14.2. For a generic $X$ in this family of $K 3$ surfaces, $\overline{\mathrm{NS}}(X)$ is spanned over $\mathbb{Z}$ by $H, E_{1}, \ldots, E_{12}$ and $L_{1}, L_{2}, L_{3}$. 
Proof. We have already demonstrated that $\overline{\mathrm{NS}}(X)$ contains $H, E_{1}, \ldots, E_{12}$, with $H^{2}=4, H \cdot E_{i}=0$ and $E_{i} \cdot E_{j}=-2 \delta_{i j}$. Therefore the rank is at least 13 . On the other hand, the moduli space has dimension $2 \cdot 2 \cdot 2 \cdot 4-\left(2^{2}-1\right) \cdot 3-4^{2}=7$. Hence the dimension of $\overline{\mathrm{NS}}(X)$ for generic $X$ must be exactly 13 .

Since $L_{i} \cdot H=4$ and $L_{i} \cdot E_{j}=0$ if $j \in\{4 i-3,4 i-2,4 i-1,4 i\}$, by comparing intersection numbers, we obtain

$$
\begin{aligned}
& L_{1}=H-\left(E_{5}+\ldots E_{12}\right) / 2, \\
& L_{2}=H-\left(E_{1}+\cdots+E_{4}+E_{9}+\cdots+E_{12}\right) / 2, \\
& L_{3}=H-\left(E_{1}+\cdots+E_{8}\right) / 2 .
\end{aligned}
$$

Note that $L_{1}+L_{2}+L_{3}$ is already in $\mathbb{Z} H+\mathbb{Z} E_{1}+\cdots+\mathbb{Z} E_{12}$.

The discriminant of the lattice $M$ spanned by $H$, the three $L_{i}$, and the twelve $E_{j}$ is $4 \cdot 2^{12} / 2^{4}=2^{10}=1024$. Since this discriminant is a power of 2 , if the NéronSeveri lattice is larger than $M$, there exists an element $D \in \mathbb{Q} H+\mathbb{Q} E_{1}+\ldots \mathbb{Q} E_{12}$ where all the denominators are powers of 2 .

In that case, we claim that $2 D \in \mathbb{Z} H+\ldots \mathbb{Z} E_{12}$. Let $2^{e}$ be the largest power of 2 in a denominator of a coefficient of $E_{i}$ in $D$. If $e \geqslant 2$, then $2^{e-2} D \cdot E_{i}$ is not an integer, since $E_{i}^{2}=-2$. Also, we cannot have $D=m H / 2^{e}+\left(c_{1} E_{1}+\right.$ $\left.\ldots c_{12} E_{12}\right) / 2$ with $c_{i}$ integers, $e \geqslant 2$ and $m$ odd, for then $2^{e-1} D=m H / 2+$ $2^{e-2}\left(c_{1} E_{1}+\ldots c_{12} E_{12}\right)$ is in $\overline{\mathrm{NS}}(X)$, and so is $m H / 2$. But this is impossible, since $(m H / 2)^{2}=m^{2}$ is odd while the intersection pairing is even. Without loss of generality (by subtracting integer multiples of $H$ and $E_{i}$ ), we may thus assume that $D=\left(c_{1} E_{1}+\cdots+c_{12} E_{12}\right) / 2$ or $D=\left(H+c_{1} E_{1}+\cdots+c_{12} E_{12}\right) / 2$, where $c_{i} \in\{0,1\}$.

In the first case, we note that $\sum c_{i} \in\{0,8,16\}$ by Lemma 2.3. Now $\sum c_{i}=$ 16 is impossible, while $\sum c_{i}=0$ is trivial. Therefore, we need to show that if $\sum c_{i}=8$, then $D$ is one of $H-L_{i}$. If not, then $D \cdot L_{3} \in \mathbb{Z}$ shows that $c_{1}+\cdots+c_{8}$ is even. It must be at least 4 (otherwise $\sum c_{i}=\left(c_{1}+\cdots+c_{8}\right)+c_{9}+\cdots+c_{12}$ would be less than 8 ) and cannot be 8 (otherwise $D=H-L_{3}$ ). Finally, it cannot be 6 (otherwise, subtracting $H-L_{3}$ would lead to a divisor $y=\left(\sum d_{i} E_{i}\right) / 2$ with $d_{i} \in\{0,1\}$ and $\sum d_{i}=4$, which is impossible). We conclude that $c_{1}+\cdots+c_{8}=4$. Similarly $c_{1}+\cdots+c_{4}+c_{5}+\cdots c_{12}=4$ and $c_{5}+\cdots+c_{12}=4$. Adding yields $\sum c_{i}=6$, which is a contradiction.

An easy discriminant calculation shows:

COROLlaRY 14.3. For a generic $X$ in this family of K3 surfaces, $\overline{\mathrm{NS}}(X)$ has a basis given by $H, L_{1}, L_{2}, L_{3}, E_{1}, E_{2}, E_{3}, E_{5}, E_{6}, E_{7}, E_{9}, E_{10}$, and $E_{11}$. 
14.4. Reverse map. Starting from the data of a $\mathrm{K} 3$ surface $X$ with line bundles $L_{1}, L_{2}, L_{3}$ and $H$ coming from a $2 \times 2 \times 2 \times 4$ box $A$, we show how to recover the box. Consider the map

$$
\mathrm{H}^{0}\left(L_{1}\right) \otimes \mathrm{H}^{0}\left(L_{2}\right) \otimes \mathrm{H}^{0}(H) \rightarrow \mathrm{H}^{0}\left(L_{1} \otimes L_{2} \otimes H\right) .
$$

The dimension of the domain is $2 \cdot 2 \cdot 4=16$. The dimension of the image can be computed by the Riemann-Roch formula, after noting that

$$
\left(L_{1}+L_{2}+H\right)^{2}=0+0+4+2 \cdot 2+2 \cdot 4+2 \cdot 4=24 .
$$

Since $L_{1}+L_{2}+H$ is the class of a big and nef divisor, an easy application of Riemann-Roch on the K3 surface $X$ yields

$$
\mathrm{H}^{0}\left(L_{1}+L_{2}+H\right)=\frac{1}{2}\left(L_{1}+L^{2}+H\right)^{2}+\chi\left(\mathcal{O}_{X}\right)=\frac{24}{2}+2=14 .
$$

Therefore, the kernel (which we will soon identify with $V_{3}^{\vee}$ ) of (39) has dimension 2, and we obtain a $2 \times 2 \times 2 \times 4$ box $B \in V_{1} \otimes V_{2} \otimes V_{3} \otimes U$, where $V_{1}=H^{0}\left(L_{1}\right)$, $V_{2}=H^{0}\left(L_{2}\right)$, and $U=H^{0}(H)$.

Let $X(B) \in \mathbb{P}\left(V_{1}^{\vee}\right) \times \mathbb{P}\left(V_{2}^{\vee}\right) \times \mathbb{P}\left(V_{3}^{\vee}\right) \times \mathbb{P}\left(U^{\vee}\right)$ denote the K3 surface associated to $B$. To see that $B$ is in fact the desired box $A$ (once $V_{3}^{\vee}$ is correctly identified with the kernel of (39)), it suffices to show that $X(B)_{12 U}$ is in fact equal to $X_{12 U}$ as sets in $\mathbb{P}\left(V_{1}^{\vee}\right) \times \mathbb{P}\left(V_{2}^{\vee}\right) \times \mathbb{P}\left(U^{\vee}\right)$. It is equivalent to show that the threefold $Y(B)_{12}$ associated to $B$ is the same as the threefold $Y_{12}$ in $\mathbb{P}\left(V_{1}^{\vee}\right) \times \mathbb{P}\left(V_{2}^{\vee}\right) \times \mathbb{P}\left(U^{\vee}\right)$, since $X(B)_{12 U}$ (and $X_{12 U}$ ) is then recovered as the ramification locus of $Y(B)_{12}=$ $Y_{12} \rightarrow \mathbb{P}\left(U^{\vee}\right)$. (In other words, if two $2 \times 2 \times 2 \times 4$ boxes yield the same threefold, then they must be the same box!) Now the equality $Y_{12} \subset Y(B)_{12}$ is true by the very construction of $B$, yielding $X_{12 U} \subset X(B)_{12 U}$. Then $X_{U} \subset X(B)_{U}$, but since both are defined by quartics, we have $X_{U}=X(B)_{U}$, and then $X_{12 U}=X(B)_{12 U}$ and also $Y_{12} \subset Y(B)_{12}$, as desired.

We have proved Theorem 14.1.

\section{5. $2 \otimes 2 \otimes \operatorname{Sym}^{2}(4)$}

In this section, we study the orbits of $V_{1} \otimes V_{2} \otimes \operatorname{Sym}^{2} V_{3}$, where $V_{1}, V_{2}$, and $V_{3}$ are $F$-vector spaces of dimensions 2, 2, and 4, respectively. We show that these orbits correspond to $\mathrm{K} 3$ surfaces lattice-polarized by a rank-2 lattice:

THEOREM 15.1. Let $V_{1}, V_{2}$, and $V_{3}$ be $F$-vector spaces of dimensions 2 , 2, and 4 , respectively. Let $G^{\prime}=\mathrm{GL}\left(V_{1}\right) \times \mathrm{GL}\left(V_{2}\right) \times \mathrm{GL}\left(V_{3}\right)$, and let $G$ be the quotient of $G^{\prime}$ by the kernel of the multiplication map $\mathbb{G}_{m} \times \mathbb{G}_{m} \times \mathbb{G}_{m} \rightarrow \mathbb{G}_{m}$ sending 
$\left(\gamma_{1}, \gamma_{2}, \gamma_{3}\right)$ to $\gamma_{1} \gamma_{2} \gamma_{3}^{2}$. Let $\Lambda$ be the lattice whose Gram matrix is

$$
\left(\begin{array}{ll}
0 & 4 \\
4 & 4
\end{array}\right)
$$

and let $S=\left\{e_{1}, e_{2}\right\}$. Then the $G(F)$-orbits of an open subset of $V(F)$ are in bijection with the $F$-rational points of an open subvariety of the moduli space $\mathcal{M}_{\Lambda, S}$ of $K 3$ surfaces $X$ lattice-polarized by $(\Lambda, S)$.

15.1. Construction of $\mathbf{K} 3$ surfaces. From a general element $A \in V_{1} \otimes V_{2} \otimes$ $\operatorname{Sym}^{2}\left(V_{3}\right)$, we obtain several natural surfaces. We view $A$ as a tridegree $(1,1,2)$ form, denoted by $A(\cdot, \cdot, \cdot)$, on $V_{1}^{\vee} \times V_{2}^{\vee} \times V_{3}^{\vee}$. First, define the quartic surface $X_{3}:=\left\{z \in \mathbb{P}\left(V_{3}^{\vee}\right): \operatorname{det} A(\cdot, \cdot, z)=0\right\}$. If $X_{3}$ is nonsingular or has only rational double point singularities, then $X_{3}$ is a K3 surface. We call such $A$ nondegenerate, and we will only consider such $A$. Now let

$$
\begin{aligned}
& X_{13}:=\left\{(x, z) \in \mathbb{P}\left(V_{1}^{\vee}\right) \times \mathbb{P}\left(V_{3}^{\vee}\right): A(x, \cdot, z)=0\right\} \\
& X_{23}:=\left\{(y, z) \in \mathbb{P}\left(V_{2}^{\vee}\right) \times \mathbb{P}\left(V_{3}^{\vee}\right): A(\cdot, y, z)=0\right\} .
\end{aligned}
$$

These are each cut out by two bidegree $(1,2)$ forms in $\mathbb{P}^{1} \times \mathbb{P}^{3}$. Note that there are natural projections $X_{i 3} \rightarrow X_{3}$ for $i=1$ or 2 , and any isolated singularities on $X_{3}$ will be blown up by these maps. Finally, we let

$$
X_{123}:=\left\{(x, y, z) \in \mathbb{P}\left(V_{1}^{\vee}\right) \times \mathbb{P}\left(V_{2}^{\vee}\right) \times \mathbb{P}\left(V_{3}^{\vee}\right): A(x, \cdot, z)=A(\cdot, y, z)=0\right\} .
$$

The surface $X_{123}$ projects to $X_{i 3}$ for $i=1$ or 2 , and we see that all of these surfaces are birational.

The projection of $X_{123}$ to $\mathbb{P}\left(V_{1}^{\vee}\right) \times \mathbb{P}\left(V_{2}^{\vee}\right)$ has degree 8 . For any point $(x, y) \in$ $\mathbb{P}\left(V_{1}^{\vee}\right) \times \mathbb{P}\left(V_{2}^{\vee}\right)$, the preimage in $X_{123}$ is the intersection of a $\mathbb{P}^{2}$ of quadrics in $\mathbb{P}\left(V_{3}^{\vee}\right)$.

For $\{i, j\}=\{1,2\}$, another way to construct $X_{i 3}$ is to view a general element of $V_{j} \otimes \operatorname{Sym}^{2}\left(V_{3}\right)$ as giving a genus one curve of degree 4 in $\mathbb{P}\left(V_{3}^{\vee}\right)$, namely the base locus of the pencil of quadrics in $\mathbb{P}\left(V_{3}^{\vee}\right)$. Then an element $v$ of $V_{i} \otimes V_{j} \otimes \operatorname{Sym}^{2}\left(V_{3}\right)$ gives a pencil over $\mathbb{P}\left(V_{i}^{\vee}\right)$ of genus one curves, and the discriminant has degree 24 and is irreducible. This gives $X_{i 3}$ as a genus one fibration over $\mathbb{P}\left(V_{i}^{\vee}\right)$ with generically only nodal reducible fibers.

Although it will not be directly relevant to the moduli problem, yet another $\mathrm{K} 3$ surface $Y$ may be obtained by viewing $v$ as a symmetric matrix of bilinear forms on $V_{1}^{\vee} \times V_{2}^{\vee}$. The determinant of this matrix is thus a bidegree $(4,4)$ curve in $\mathbb{P}\left(V_{1}^{\vee}\right) \times \mathbb{P}\left(V_{2}^{\vee}\right)$, and the double cover of $\mathbb{P}\left(V_{1}^{\vee}\right) \times \mathbb{P}\left(V_{2}^{\vee}\right)$ ramified at this bidegree $(4,4)$ curve is a $\mathrm{K} 3$ surface. It is also a genus one fibration over both 
$\mathbb{P}\left(V_{1}^{\vee}\right)$ and over $\mathbb{P}\left(V_{2}^{\vee}\right)$. Indeed, as a fibration over $\mathbb{P}\left(V_{i}^{\vee}\right)$, the smooth irreducible fibers are genus one curves of degree 2 (namely, double covers of $\mathbb{P}\left(V_{j}^{\vee}\right)$ ramified at a degree 4 subscheme of $\mathbb{P}\left(V_{j}^{\vee}\right)$ ).

15.2. Néron-Severi lattice. The $\mathrm{K} 3$ surface $X_{123}$ corresponding to a very general point in the moduli space has rank 2 , since it is a genus one fibration without any extra divisors. The Néron-Severi lattice is spanned by $L_{1}, L_{2}$, and $L_{3}$ (the pullback of hyperplane divisors from $\mathbb{P}\left(V_{1}^{\vee}\right), \mathbb{P}\left(V_{2}^{\vee}\right)$ and $\mathbb{P}\left(V_{3}^{\vee}\right)$ ) which have the intersection numbers $L_{i}^{2}=0$ and $L_{i} \cdot L_{3}=4$ for $i=1$ or $2, L_{1} \cdot L_{2}=8$, and $L_{3}^{2}=4$. It is easily seen that $2 L_{3}=L_{1}+L_{2}$, so the lattice spanned by their classes in the Néron-Severi group has a basis $\left\{L_{1}, L_{3}\right\}$, with intersection matrix

$$
\left(\begin{array}{ll}
0 & 4 \\
4 & 4
\end{array}\right)
$$

In fact, it must be the entire Néron-Severi lattice. To prove this, it suffices to show that the three classes $L_{1} / 2, L_{3} / 2$ and $\left(L_{1}+L_{3}\right) / 2$ do not arise from divisors on the surface. The first assertion is immediate, since $L_{1}$ is the class of an elliptic fiber, and therefore not multiple. The other two $\mathbb{Q}$-divisor classes have odd selfintersection, so they cannot come from divisors, either.

15.3. Moduli problem. To prove Theorem 15.1, we need to construct an element of $V_{1} \otimes V_{2} \otimes \operatorname{Sym}^{2}\left(V_{3}\right)$ from a K3 surface $X$ with divisors $L_{1}$ and $L_{3}$ that have intersection matrix (40).

Proof of Theorem 15.1. First, the natural multiplication map $\operatorname{Sym}^{2} \mathrm{H}^{0}\left(X, L_{3}\right) \rightarrow$ $\mathrm{H}^{0}\left(X, 2 L_{3}\right)$ is an isomorphism by a dimension count (each has dimension 10$)$. We consider the multiplication map

$\mu: \mathrm{H}^{0}\left(X, L_{1}\right) \otimes \operatorname{Sym}^{2} \mathrm{H}^{0}\left(X, L_{3}\right) \stackrel{\cong}{\rightrightarrows} \mathrm{H}^{0}\left(X, L_{1}\right) \otimes \mathrm{H}^{0}\left(X, 2 L_{3}\right) \rightarrow \mathrm{H}^{0}\left(X, L_{1}+2 L_{3}\right)$.

The dimension of the domain is 20 , and $\mathrm{h}^{0}\left(X, L_{1}+2 L_{3}\right)=\frac{1}{2}\left(L_{1}+2 L_{3}\right)^{2}+$ $\chi\left(\mathcal{O}_{X}\right)=16+2=18$. We claim that (41) is surjective, in which case the kernel is 2-dimensional and will give the desired tensor.

The surjectivity of $\mu$ follows directly from the basepoint-free pencil trick and the fact that $\mathrm{H}^{1}\left(X, L_{1}^{-1} \otimes L_{3}^{\otimes 2}\right)$ is 0 . This last vanishing may be obtained by computing $\chi\left(L_{1}^{-1} \otimes L_{3}^{\otimes 2}\right)=2, \mathrm{~h}^{0}\left(X, L_{1}^{-1} \otimes L_{3}^{\otimes 2}\right)=2$ (because the bundle is nef and semiample), and $\mathrm{h}^{2}\left(X, L_{1}^{-1} \otimes L_{3}^{\otimes 2}\right)=0$ by Serre duality. Note that the basepoint-free pencil trick also gives an isomorphism of the kernel of $\mu$ with $\mathrm{H}^{0}\left(X, L_{1}^{-1} \otimes L_{3}^{\otimes 2}\right)$. 
The usual argument (for example, see the proofs of Theorems 4.1 and 7.1) shows that these two constructions are inverse to one another.

\section{6. $\operatorname{Sym}^{2}(2) \otimes \operatorname{Sym}^{2}(4)$}

Finally, just as in the Rubik's revenge and penteract cases, we may consider a symmetric linear subspace of the previous case of $2 \otimes 2 \otimes \mathrm{Sym}^{2}(4)$. Specifically, let $V=\operatorname{Sym}^{2}\left(V_{1}\right) \otimes \operatorname{Sym}^{2}\left(V_{2}\right)$, where $V_{1}$ and $V_{2}$ are $F$-vector spaces of dimensions 2 and 4, respectively. Then the general orbits of $V$ under linear transformations on $V_{1}$ and $V_{2}$ correspond to certain $\mathrm{K} 3$ surfaces of rank at least 9 over $\bar{F}$ :

THEOREM 16.1. Let $V_{1}$ and $V_{2}$ be $F$-vector spaces of dimensions 2 and 4 , respectively. Let $G^{\prime}=\mathbb{G}_{m} \times \mathrm{GL}\left(V_{1}\right) \times \mathrm{GL}\left(V_{2}\right)$ and let $G$ be its quotient by the kernel of the multiplication map $\mathbb{G}_{m} \times \mathbb{G}_{m} \times \mathbb{G}_{m} \rightarrow \mathbb{G}_{m}$ sending $\left(\gamma_{1}, \gamma_{2}, \gamma_{3}\right)$ to $\gamma_{1} \gamma_{2}^{2} \gamma_{3}^{2}$. Let $\Lambda$ be the lattice whose Gram matrix is

$$
\left(\begin{array}{ccccccccc}
0 & 4 & 1 & 1 & 1 & 1 & 1 & 1 & 1 \\
4 & 4 & 0 & 0 & 0 & 0 & 0 & 0 & 0 \\
1 & 0 & -2 & 0 & 0 & 0 & 0 & 0 & 0 \\
1 & 0 & 0 & -2 & 0 & 0 & 0 & 0 & 0 \\
1 & 0 & 0 & 0 & -2 & 0 & 0 & 0 & 0 \\
1 & 0 & 0 & 0 & 0 & -2 & 0 & 0 & 0 \\
1 & 0 & 0 & 0 & 0 & 0 & -2 & 0 & 0 \\
1 & 0 & 0 & 0 & 0 & 0 & 0 & -2 & 0 \\
1 & 0 & 0 & 0 & 0 & 0 & 0 & 0 & -2
\end{array}\right)
$$

and let $S=\left\{e_{1}, e_{2}\right\}$. Then the $G(F)$-orbits of an open subset of $V(F)$ are in bijection with the $F$-rational points of an open subvariety of the moduli space $\mathcal{M}_{\Lambda, S}$ of $K 3$ surfaces $X$ lattice-polarized by $(\Lambda, S)$.

We view this vector space as a subspace of $V_{1} \otimes V_{1} \otimes \operatorname{Sym}^{2}\left(V_{2}\right)$. Then the K3 surfaces in this case are constructed in the same way as in Section 15. However, for a general element $A \in \operatorname{Sym}^{2}\left(V_{1}\right) \otimes \operatorname{Sym}^{2}\left(V_{2}\right)$, we obtain eight rank singularities (over $\bar{F}$ ) on the surface $X_{3} \subset \mathbb{P}\left(V_{2}^{\vee}\right)$; they are exactly the points where the symmetric $2 \times 2$ matrix of quadratic forms $A(\cdot, \cdot, z)$ is identically zero, namely the intersection of three quadrics in $\mathbb{P}^{3}$. These singularities are blown up in the other surfaces $X_{12}, X_{13}$, and $X_{123}$ described in Section 15, all of which are isomorphic nonsingular K3 surfaces for the general $A$. Let $E_{i}$ for $1 \leqslant i \leqslant 8$ denote these exceptional divisors on $X:=X_{123}$.

The symmetry also shows that the line bundles $L_{1}$ and $L_{2}$, defined as pullbacks of $\mathcal{O}_{\mathbb{P}\left(V_{1}^{\vee}\right)}(1)$ to $X$, are the same. We thus have the relation 


$$
2 L_{3}=2 L_{1}+\sum_{i} E_{i}
$$

where $L_{3}$ is the pullback of $\mathcal{O}_{\mathbb{P}\left(V_{2}^{\vee}\right)}(1)$ to $X$. Computing the intersection numbers of $L_{1}, L_{3}$, and the $E_{i}$ in the usual way gives the intersection matrix (42) (with respect to the basis $L_{1}, L_{3}, E_{1}, \ldots, E_{7}$ ). The lattice spanned by these divisors has rank 9 and discriminant 256 . To check that it is all of $\overline{\mathrm{NS}}(X)$, we observe by direct calculation that any element of the dual lattice has the form

$$
D=\frac{c L_{3}}{4}+\frac{1}{2} \sum_{i=1}^{7} d_{i} E_{i} .
$$

First, observe that $c$ cannot be odd; otherwise, $2 D$ and hence $c L_{3} / 2$ would be in $\overline{\mathrm{NS}}(X)$, which is impossible since it has odd self-intersection. We can therefore write

$$
D=\frac{c L_{3}}{2}+\frac{1}{2} \sum_{i=1}^{7} d_{i} E_{i}
$$

Then by symmetry,

$$
D^{\prime}=\frac{c L_{3}}{2}+\frac{1}{2} \sum_{i=1}^{6} d_{i} E_{i}+d_{7} E_{8}
$$

is also in $\overline{\mathrm{NS}}(X)$. Subtracting, we get $d_{7}\left(E_{7}-E_{8}\right) / 2 \in \overline{\mathrm{NS}}(X)$, which is impossible by Lemma 2.3, unless $d_{7}$ is even. Similarly, all the $d_{i}$ are even, resulting in $D=c L_{3} / 2$, which is impossible by the argument above.

To complete the proof of Theorem 16.1, we check that a K3 surface $X$ whose Néron-Severi lattice contains the lattice (42) may be obtained from an element of our vector space $V$. The construction in the proof of Theorem 15.1 applies here, and we only need to check that the resulting element $A$ is symmetric in the two 2-dimensional vector spaces. This is by the same argument as in the symmetric penteract cases: by Theorem 15.1 , the tridegree $(1,1,2)$ form $A$ in $U_{1} \otimes U_{2} \otimes$ $\operatorname{Sym}^{2}\left(U_{3}\right)$ gives a K3 surface whose two projections to $\mathbb{P}\left(U_{1}^{\vee}\right)$ and $\mathbb{P}\left(U_{2}^{\vee}\right)$ are identical (under some identification $\phi: U_{2} \rightarrow U_{1}$ ). Therefore, if $A$ is viewed as a $2 \times 2$ matrix $B=\left(b_{i j}\right)$ of quadratic forms on the 4-dimensional space $U_{3}$, we must have that $b_{12}=b_{21}$ identically, or in other words, the image of $A$ under $\operatorname{Id} \otimes \phi \otimes \operatorname{Id}$ is an element of $\operatorname{Sym}^{2}\left(U_{1}\right) \otimes \operatorname{Sym}^{2}\left(U_{3}\right)$.

\section{Applications and connections}

In this section, we prove Theorems 1.5 and 1.6, as well as several related results, by using hyperdeterminants and the automorphisms of the $\mathrm{K} 3$ surfaces discussed in earlier sections. 
17.1. Definition of hyperdeterminant. The hyperdeterminant of a multidimensional matrix is a natural analogue of the determinant of a square matrix. It was first introduced by Cayley [24, pages 80-94], while a detailed study was carried out in the important work of Gelfand et al. [35].

We may define the hyperdeterminant as follows (see [35] for more details). Let $F$ be a field, and let $T: V_{1} \otimes \cdots \otimes V_{r} \rightarrow F$ be a linear map, where $V_{1}, \ldots, V_{r}$ are $F$-vector spaces having dimensions $k_{1}+1, \ldots, k_{r}+1$, respectively. By choosing bases for $V_{1}, \ldots, V_{r}$, we may view $T$ as a $\left(k_{1}+1\right) \times \cdots \times\left(k_{r}+1\right)$ matrix. The kernel $\operatorname{ker}(T)$ of $T$ is defined to be

$\left\{v=v_{1} \otimes \cdots \otimes v_{r} \in V_{1} \otimes \cdots \otimes V_{r}: T\left(v_{1}, \ldots, v_{i-1}, \cdot, v_{i+1}, \ldots, v_{r}\right)=0\right.$ for all $\left.i\right\}$

By definition, a hyperdeterminant $\operatorname{det}(T)$ of the multidimensional matrix $T$ is a polynomial of minimal degree in the entries of $T$ whose vanishing is equivalent to $T$ having a nontrivial kernel. If it exists, the hyperdeterminant is then well defined up to a scalar multiple.

The necessary conditions on the dimensions of the matrix $T$ for the existence of hyperdeterminants was determined by Gelfand et al. [35, Ch. 14]:

THEOREM 17.1 [35]. Assume without loss of generality that $k_{r} \geqslant k_{1}, \ldots, k_{r-1}$. Then hyperdeterminants exist for $\left(k_{1}+1\right) \times \cdots \times\left(k_{r}+1\right)$ matrices if and only if $k_{r} \leqslant k_{1}+\cdots+k_{r-1}$.

For example, when $r=2$, hyperdeterminants exist if and only if $k_{1}=k_{2}$, that is, the matrix is square. By definition, we see that a square matrix $T$ has vanishing hyperdeterminant if and only if $T$ has a nontrivial left (equivalently, right) kernel. Thus the hyperdeterminant in this case coincides with the usual determinant.

\subsection{Interpretations in terms of fixed-point-free automorphisms.} Although interpretations of the determinant of a square matrix (for example, as a volume) have been known for centuries, interpretations for the hyperdeterminant for higher-dimensional matrices have been less forthcoming.

In [8], an interpretation of the hyperdeterminant in the case of a $2 \times 2 \times 2$ matrix was given, namely, as the discriminant of an associated quadratic algebra. Analogous interpretations for the hyperdeterminant of a $2 \times 3 \times 3$ matrix-namely, as the discriminant of an associated cubic algebra-were given in [9]. In the works $[4,14,16]$, orbits on multidimensional matrices of various dimensions were shown to be in bijection with certain data involving algebraic curves, and in these cases the hyperdeterminants are equal to the discriminants of the corresponding curves. Thus the nonvanishing of the hyperdeterminant in these cases corresponds 
to the nondegeneracy of the associated rings and the nonsingularity of the associated curves, respectively.

For the orbit parametrizations of K3 surfaces by multidimensional matrices that we have studied in this paper, we find that the hyperdeterminant does not coincide with the discriminant, but only divides it. This raises the question as to the interpretation of the hyperdeterminant in these cases. In the cases of $4 \times 4 \times 4$ and $2 \times 2 \times 2 \times 2 \times 2$ matrices, we showed that the generic orbits of such matrices correspond to $\mathrm{K} 3$ surfaces with at most isolated double point singularities that are $(\Lambda, S)$-polarized for some pair $(\Lambda, S)$. Moreover, these K3 surfaces are naturally equipped with birational automorphisms $\Phi$, which lift to automorphisms of the nonsingular models; these birational automorphisms are in fact automorphisms whenever the associated $\mathrm{K} 3$ surfaces have no rank singularities.

The interpretation of the hyperdeterminant locus that we obtain in this case is then as follows:

THEOREM 17.2. Let $T$ be a $4 \times 4 \times 4$ matrix, and suppose that the associated $K 3$ surfaces $X_{1}, X_{2}, X_{3}$ via Theorem 4.1 have no rank singularities. Then the hyperdeterminant of $T$ vanishes if and only if the associated automorphism $\Phi$ of $X=X_{1}$ has a fixed point.

Proof. Suppose the hyperdeterminant of $T$ vanishes, and let $v_{1} \otimes v_{2} \otimes v_{3} \in \operatorname{ker}(T)$. For each $i \in\{1,2,3\}$, let $\bar{v}_{i}$ denote the image of $v_{i}$ in $\mathbb{P}\left(V_{i}\right)$. Then $\bar{v}_{i}$ is a point on $X_{i}$. By the definition of $\Phi$ and the fact that there are no rank singularities on the $X_{i}$, we see that $\psi_{12}\left(\bar{v}_{1}\right)=\bar{v}_{2}, \psi\left(\bar{v}_{2}\right)=\bar{v}_{3}$, and $\psi\left(\bar{v}_{3}\right)=\bar{v}_{1}$; hence $\Phi\left(\bar{v}_{1}\right)=\bar{v}_{1}$, yielding a fixed point of $\Phi$ on $X_{1}$, as desired.

If some of the $X_{i}$ have isolated rank singularities, then the maps $\psi_{i j}: X_{i}-\rightarrow$ $X_{j}$ are not isomorphisms but birational maps. These maps lift uniquely to isomorphisms $\tilde{\psi}_{i j}: \tilde{X}_{i} \rightarrow \tilde{X}_{j}$ between the nonsingular models $\tilde{X}_{i}$ and $\tilde{X}_{j}$ of $X_{i}$ and $X_{j}$, respectively (see, for example, [3, Theorem 10.21]). We thus obtain an automorphism $\tilde{\Phi}=\tilde{\psi}_{31} \circ \tilde{\psi}_{23} \circ \tilde{\psi}_{12}$ of $X=X_{1}$. In this case too, we may still use the hyperdeterminant to detect fixed points of $\tilde{\Phi}$ :

THEOREM 17.3. Let $T$ be a $4 \times 4 \times 4$ matrix, and suppose that the associated K3 surfaces $X_{1}, X_{2}, X_{3}$ via Theorem 4.1 have only isolated double point singularities. If the associated automorphism $\tilde{\Phi}$ of the nonsingular model $\tilde{X}=\tilde{X}_{1}$ of $X_{1}$ has a fixed point, then the hyperdeterminant of $T$ vanishes.

Proof. Suppose $\tilde{v}_{1}$ on $\tilde{X}_{1}$ is a fixed point of $\tilde{\Phi}$. Let $\tilde{v}_{2}=\tilde{\psi}_{12}\left(\tilde{v}_{1}\right)$ and $\tilde{v}_{3}=\tilde{\psi}_{23}\left(\tilde{v}_{2}\right)$, so that $\tilde{v}_{1}=\tilde{\psi}_{31}\left(\tilde{v}_{3}\right)$. Let $v_{1}, v_{2}, v_{3}$ denote the images of $\tilde{v}_{1}, \tilde{v}_{2}, \tilde{v}_{3}$ in $X_{1}, X_{2}, X_{3}$, 
respectively. We claim that $v_{1} \otimes v_{2} \otimes v_{3} \in \operatorname{ker}(T)$. Indeed, the nonsingularization map $\tilde{X}_{1} \rightarrow X_{1}$ factors through

$$
X_{12}=\left\{(x, y) \in \mathbb{P}\left(V_{1}^{\vee}\right) \times \mathbb{P}\left(V_{2}^{\vee}\right): A(x, y, \cdot)=0\right\} .
$$

(In fact, $X_{12}$ is isomorphic to $\tilde{X}_{1}$ when $X_{1}$ only has simple isolated rank singularities.) It follows that $T\left(v_{1}, v_{2}, \cdot\right)=0$. Similarly, $T\left(\cdot, v_{2}, v_{3}\right)=T\left(v_{1}\right.$, $\left.\cdot, v_{3}\right)=0$. This is the desired conclusion.

In particular, if the hyperdeterminant is nonzero, then the automorphism $\tilde{\Phi}$ of $X$ has no fixed points.

Similarly, we have:

THEOREM 17.4. Let $T$ be $a 2 \times 2 \times 2 \times 2 \times 2$ matrix, and suppose that the associated $K 3$ surfaces $X_{i j k}$ via Theorem 7.1 have no rank singularities. Then the hyperdeterminant of $T$ vanishes if and only if one (equivalently, every one) of the associated automorphisms $\Phi_{a b c d e}$ of $X_{i j k}$ has a fixed point.

THEOREM 17.5. Let $T$ be a $2 \times 2 \times 2 \times 2 \times 2$ matrix, and suppose that the associated K3 surfaces $X_{i j k}$ via Theorem P7.1 have only isolated double point singularities. If, for any $i, j, k$, the associated automorphism $\tilde{\Phi}_{\text {abcde }}$ of the nonsingular model $\tilde{X}=\tilde{X}_{i j k}$ of $X_{i j k}$ has a fixed point, then the hyperdeterminant of $T$ vanishes.

The proofs are similar to those of Theorems 17.2 and 17.3.

In Sections 17.3 and 17.4, we use these theorems about hyperdeterminants vanishing to exhibit fixed-point-free automorphisms of finite order and of positive entropy, respectively, for most of the $\mathrm{K} 3$ surfaces in some of the families we consider (namely, those where the hyperdeterminant does not vanish).

17.3. Fixed-point-free automorphisms of finite order. We may use Theorems 17.3 and 17.5 to find fixed-point-free automorphisms of finite order for most of the K3 surfaces in some of the symmetric Rubik's revenge and penteract families.

For the doubly symmetric Rubik's revenge case, the automorphism $\Phi$ of a general member $X$ of the family of K3 surfaces gives an involution of $X$ (as described in Section 5.3). When the hyperdeterminant does not vanish, by Theorem 17.3, this involution is fixed-point-free. Such an involution produces an Enriques surface, so the moduli space of the K3s in this family also correspond to (an open part of the) moduli space for certain Enriques surfaces. 
Similarly, for the triply symmetric Rubik's revenge, the automorphism $\Phi$ is a fixed-point-free involution for the general Hessian quartic surface; this involution is studied in [32].

For quadruply and quintuply symmetric penteracts, recall from Sections 12.2 and 13.2 that the 5-cycles $\Phi_{i j k l m}$ are order 4 automorphisms. By Theorem 17.5, these automorphisms are fixed-point-free. As discussed in Sections 12.2 and 13.2, the square of each of these automorphisms is an involution but no longer fixedpoint-free.

Note that we have previously constructed other automorphisms for the penteract (and symmetric penteract) cases with finite order but which are not fixed-point-free. For example, for the triply symmetric penteracts, the four-cycles

$$
\alpha_{23,5} \circ \alpha_{34,5}: X_{1234} \rightarrow X_{1235} \rightarrow X_{1245} \rightarrow X_{1345}
$$

and $\alpha_{13,5} \circ \alpha_{34,5}$ are order 3 . Viewing the K3 surface $X_{1234}$ as a genus one fibration over $\mathbb{P}\left(V_{1}^{\vee}\right)$ (respectively, $\mathbb{P}\left(V_{2}^{\vee}\right)$ ), the automorphism $\alpha_{23,5} \circ \alpha_{34,5}$ (respectively, $\left.\alpha_{13,5} \circ \alpha_{34,5}\right)$ is given by translation by a 3-torsion section of the Jacobian fibration (see [16, Section 6.3.2]). The reducible fibers of the genus one fibration have fixed points, however. Similar automorphisms (corresponding to translations by 3torsion sections of the Jacobian fibrations) appear for the doubly triply, quadruply, and quintuply symmetric penteracts as well.

17.4. Fixed-point-free automorphisms of positive entropy. We show that many of the automorphisms that we have constructed in earlier sections have positive entropy and are fixed-point-free for the general member of the corresponding family. Specifically, we obtain such fixed-point-free automorphisms with positive entropy for the cases from lines $6,9,11,13$, 15, and 16 of Table 1.

In each of these cases, by the parametrization theorems in this paper, the NéronSeveri lattice of the K3 surfaces $X$ (over $\bar{F}$ ) in the families contain a given lattice $\Lambda$; the Néron-Severi lattice of the very general member of the family will be exactly $\Lambda$. We will describe the action of a particular automorphism $\Phi$ on $X$ (defined over $F$ ); we find that $\Phi^{*}$ acts on $\Lambda$ by a matrix $M$, which has an eigenvalue $\lambda$ of norm larger than 1 . Since the action of $\Phi^{*}$ on $\overline{\mathrm{NS}}(X) \otimes \mathbb{R}$ has at most one eigenvalue of modulus larger than 1 (see [21, Section 2.3.2]) and $M$ fixes the subspace $\Lambda \otimes \mathbb{R}$ in $\overline{\mathrm{NS}}(X) \otimes \mathbb{R}$, the spectral radius of $\Phi^{*}$ is exactly $\lambda$.

In other words, for each of these cases, we find that the entropy of the automorphism for each K3 surface is the logarithm of the norm of the largest eigenvalue $\lambda$ of $M$. The theorems from Section 17.2 imply that these automorphisms are fixed-point-free for the general member of the family, 
specifically when the hyperdeterminant of the corresponding element does not vanish.

Rubik's revenge. As mentioned in Section 4.4, for each $\mathrm{K} 3$ surface $X$ arising from a Rubik's revenge, there exists an automorphism $\Phi$ whose induced action on (the known part of) $\mathrm{NS}(X)$ is given by the matrix

$$
\left(\begin{array}{cc}
-3 & -8 \\
8 & 21
\end{array}\right)
$$

The characteristic polynomial of this matrix is $\lambda^{2}-18 \lambda+1$, and the largest eigenvalue is $\lambda_{\mathrm{RR}}=9+4 \sqrt{5}$. The entropy of the automorphism $\Phi$ is thus $6 \log (1+\sqrt{5}) / 2$, and by Theorem 17.2 , this automorphism is also fixed-pointfree if the hyperdeterminant of the Rubik's revenge does not vanish. This gives the proof of Theorem 1.5, an extension of Oguiso's result from [61].

Penteracts. Recall from Section 7.4 that we defined an automorphism $\Phi_{51234}$ (as a certain 5-cycle along the 1-dimensional boundary of a 5-cell), and $\Phi_{51234}$ induces the action of the matrix

$$
\left(\begin{array}{cccc}
-1 & 0 & 2 & 2 \\
-2 & 1 & 2 & 4 \\
-4 & 2 & 5 & 6 \\
-6 & 2 & 8 & 11
\end{array}\right)
$$

on (the known part of) $\mathrm{NS}(X)$. The characteristic polynomial of this matrix is $\lambda^{4}-16 \lambda^{3}+14 \lambda^{2}-16 \lambda+1$, and the maximum eigenvalue $\lambda_{\text {pent }}$ is approximately 15.1450744834468 . Therefore, the entropy of $\Phi_{51234}$ is $\log \lambda_{\text {pent }} \approx 2.717675362$. The same numerics occur for all of the other 5-cycles, by symmetry.

By Theorem 17.4, the automorphism $\Phi_{51234}$ will be fixed-point-free if the hyperdeterminant of the penteract does not vanish. Thus, we have produced a family of K3 surfaces whose general member has several fixed-point-free automorphisms with positive entropy, giving the proof of Theorem 1.6.

Recall that for the penteract (and the symmetric) cases, one may also consider automorphisms that are 3- or 4-cycles along the boundary of the 5-cell; these all have zero entropy. There are also infinitely many other automorphisms (for instance, by taking arbitrary words in the generators) with positive entropy (see Section 7.4). This is also true for the symmetric penteract cases below.

Doubly symmetric penteracts. Recall from Section 8.3 that the automorphism $\Phi_{54321}$ on a K3 surface $X$ here induces the action of the matrix (18) on (the known part of) $\overline{\mathrm{NS}}(X)$, which has characteristic polynomial 
$(\lambda-1)^{6}(\lambda+1)\left(\lambda^{2}-6 \lambda+1\right)$ and largest eigenvalue $3+2 \sqrt{2}$. The entropy of $\Phi_{54321}$ here is thus $\log (3+2 \sqrt{2})=2 \log (\sqrt{2}+1)$.

In addition, the entropy of $\Phi_{53421}$ is $\log \lambda_{\text {pent }}$, since its action on $\overline{\mathrm{NS}}(X)$ is similar to the action of the penteract case. The 5-cycles thus have entropy either $2 \log (\sqrt{2}+1)$ or $\log \lambda_{\text {pent }}$.

By Theorem 17.5, we thus find that the general member of this family of K3 surfaces has many fixed-point-free automorphisms with positive entropy.

Triply symmetric penteracts. In Section 9.3, for the general member $X$ of the family of $\mathrm{K} 3$ surfaces related to triply symmetric penteracts, we found an automorphism $\Phi_{54123}$ whose action on (the known part of) $\overline{\mathrm{NS}}(X)$ is given by the matrix (20). This matrix has characteristic polynomial $(\lambda+1)^{2}\left(\lambda^{2}-\lambda+1\right)^{5}\left(\lambda^{2}-\right.$ $3 \lambda+1)$ and largest eigenvalue $(3+\sqrt{5}) / 2=((\sqrt{5}+1) / 2)^{2}$.

As before, some of the other 5-cycles have the same numerics (by symmetry). The other 5-cycles (like $\left.\Phi_{54132}\right)$ have entropy at least $\log (3+2 \sqrt{2})$, as their action on Néron-Severi is much like that of $\Phi_{54321}$ in the doubly symmetric penteract case.

Thus, applying Theorem 17.5, we have that the general member of this family of K3 surfaces has fixed-point-free automorphisms with entropy $2 \log ((\sqrt{5}+1) / 2)$ and $2 \log (\sqrt{2}+1)$.

Doubly doubly symmetric penteracts. In Section 10.2, we described a 5-cycle automorphism $\Phi_{53214}$ on the general member of the family of K3 surfaces arising from doubly doubly symmetric penteracts. Its action on (the known part of) $\overline{\mathrm{NS}}(X)$ is given by $(24)$, with characteristic polynomial $(\lambda+1)^{12}\left(\lambda^{2}+1\right)\left(\lambda^{2}-\right.$ $4 \lambda+1)$ and largest eigenvalue $2+\sqrt{3}$.

Therefore, Theorem 17.5 implies that the general member of this family of K3 surfaces has a fixed-point-free automorphism with entropy $\log (2+\sqrt{3})$. In addition, from the automorphisms $\Phi_{53421}$ and $\Phi_{53241}$ (and other analogous 5cycles), we also obtain fixed-point-free automorphisms with entropy equal to $\log \lambda_{\text {pent }}$ and $2 \log (\sqrt{2}+1)$, respectively.

Doubly triply symmetric penteracts. The automorphism $\Phi_{53214}$ from Section 11.2, applied to the general member $X$ of the family of K3 surfaces coming from doubly triply symmetric penteracts, acts on (the known part of) $\overline{\mathrm{NS}}(X)$ by (29). It has characteristic polynomial $(\lambda-1)^{3}(\lambda+1)\left(\lambda^{2}-3 \lambda+1\right)\left(\lambda^{2}+\lambda+1\right)^{8}$ and largest eigenvalue $(3+\sqrt{5}) / 2$ (just as in the triply symmetric penteract case). The same argument shows that this gives fixed-point-free automorphisms with entropy $2 \log ((\sqrt{5}+1) / 2)$. Moreover, the automorphism $\Phi_{52413}$ is fixed-point-free with entropy $2 \log (\sqrt{2}+1)$. 


\section{Acknowledgments}

We thank Noam Elkies, Dick Gross, Curt McMullen, Bjorn Poonen, Igor Rivin, Peter Sarnak, and Ichiro Shimada for many helpful conversations. We are very grateful to Serge Cantat and Genya Zaytman for careful readings of earlier drafts and many useful comments. We also thank Arthur Baragar, Igor Dolgachev, and Brendan Hassett for feedback on earlier drafts. Finally, we are very thankful to the anonymous referees for careful readings of the paper, and for many insightful remarks and detailed references that improved it. MB was supported by a Simons Investigator Grant and NSF grant DMS-1001828. WH was supported by NSF grant DMS-1406066. AK was supported in part by National Science Foundation grant DMS-0952486 and by a grant from the MIT Solomon Buchsbaum Research Fund.

\section{References}

[1] Société Mathématique de France, Géométrie des surfaces K3: modules et périodes (Paris, 1985), Papers from the seminar held in Palaiseau, October 1981-January 1982, Astérisque No. 126 (1985).

[2] E. Arbarello, M. Cornalba, P. A. Griffiths and J. Harris, Geometry of Algebraic Curves. Vol I, Grundlehren der Mathematischen Wissenschaften [Fundamental Principles of Mathematical Sciences], 267 (Springer, New York, 1985).

[3] L. Bădescu, Algebraic Surfaces (Universitext, Springer, New York, 2001), Translated from the 1981 Romanian original by Vladimir Maşek and revised by the author.

[4] A. Beauville, 'Determinantal hypersurfaces', Michigan Math. J. 48 (2000), 39-64. Dedicated to William Fulton on the occasion of his 60th birthday.

[5] A. Beauville, 'Fano threefolds and $K 3$ surfaces', in The Fano Conference (Univ. Torino, 2004), 175-184. Turin.

[6] N. Bergeron, Z. Li, J. Millson and C. Moeglin, 'The Noether-Lefschetz conjecture and generalizations', Preprint, 2014, http://arxiv.org/abs/1412.3774.

[7] M. Bhargava, 'Higher composition laws. I. A new view on Gauss composition, and quadratic generalizations', Ann. of Math. (2) 159(1) (2004), 217-250.

[8] M. Bhargava, 'Higher composition laws II. On cubic analogues of Gauss composition', Ann. of Math. (2) 159(2) (2004), 865-886.

[9] M. Bhargava, 'Higher composition laws. III. The parametrization of quartic rings', Ann. of Math. (2) 159(3) (2004), 1329-1360.

[10] M. Bhargava, 'The density of discriminants of quartic rings and fields', Ann. of Math. (2) 162(2) (2005), 1031-1063.

[11] M. Bhargava, 'Higher composition laws IV. The parametrization of quintic rings', Ann. of Math. (2) 167(1) (2008), 53-94.

[12] M. Bhargava, 'The density of discriminants of quintic rings and fields', Ann. of Math. (2) 172(3) (2010), 1559-1591.

[13] M. Bhargava, 'Most hyperelliptic curves over $\mathbb{Q}$ have no rational points', Preprint, 2013, http://arxiv.org/abs/1308.0395.

[14] M. Bhargava, B. Gross and X. Wang, 'Pencils of quadrics and the arithmetic of hyperelliptic curves', J. Amer. Math. Soc. to appear, http://arxiv.org/abs/1310.7692. 
[15] M. Bhargava and B. H. Gross, 'The average size of the 2-selmer group of Jacobians of hyperelliptic curves having a rational weierstrass point', in Automorphic Representations and L-Functions, Tata Institute of Fundamental Research Studies in Mathematics, 22 (Tata Inst. Fund. Res., Mumbai, 2013), 23-91.

[16] M. Bhargava and W. Ho, 'Coregular spaces and genus one curves', Cambridge J. Math. 4(1) (2016), 1-119.

[17] M. Bhargava and A. Shankar, 'Binary quartic forms having bounded invariants, and the boundedness of the average rank of elliptic curves', Ann. of Math. (2) 181(1) (2015), 191-242.

[18] M. Bhargava and A. Shankar, 'Ternary cubic forms having bounded invariants, and the existence of a positive proportion of elliptic curves having rank 0', Ann. of Math. (2) 181(2) (2015), 587-621.

[19] M. Bhargava, A. Shankar and J. Tsimerman, 'On the Davenport-Heilbronn theorems and second order terms', Invent. Math. 193(2) (2013), 439-499.

[20] S. Cantat, 'Dynamique des automorphismes des surfaces K3', Acta Math. 187(1) (2001), $1-57$.

[21] S. Cantat, 'Dynamics of automorphisms of compact complex surfaces', in Frontiers in Complex Dynamics: In Celebration of John Milnor's 80th Birthday (eds. S. Sutherland, A. Bonifant and M. Lyubich) (Princeton University Press, Princeton, NJ, 2014), 463-514.

[22] J. W. S. Cassels, Rational Quadratic Forms, London Mathematical Society Monographs, 13 (Academic Press Inc. [Harcourt Brace Jovanovich Publishers], London, 1978).

[23] A. Cayley, 'A memoir on quartic surfaces', Proc. Lond. Math. Soc. 3 (1869/70), 19-69. [Collected Papers, VII, 133-181].

[24] A. Cayley, The Collected Mathematical Papers. Volume 1, Cambridge Library Collection (Cambridge University Press, Cambridge, 2009), Reprint of the 1889 original.

[25] F. Cossec and I. Dolgachev, 'On automorphisms of nodal Enriques surfaces', Bull. Amer. Math. Soc. (N.S.) 12(2) (1985), 247-249.

[26] F. R. Cossec, 'Reye congruences', Trans. Amer. Math. Soc. 280(2) (1983), 737-751.

[27] E. Dardanelli and B. van Geemen, 'Hessians and the Moduli space of cubic surfaces', in Algebraic Geometry, Contemporary Mathematics, 422 (American Mathematical Society, Providence, RI, 2007), 17-36.

[28] H. Davenport and H. Heilbronn, 'On the density of discriminants of cubic fields', Bull. Lond. Math. Soc. 1 (1969), 345-348.

[29] B. N. Delone and D. K. Faddeev, The Theory of Irrationalities of the Third Degree, Translations of Mathematical Monographs, 10 (American Mathematical Society, Providence, RI, 1964).

[30] I. V. Dolgachev, 'Mirror symmetry for lattice polarized $K 3$ surfaces', J. Math. Sci. 81(3) (1996), 2599-2630. Algebraic Geometry, Vol. 4.

[31] I. V. Dolgachev, Classical Algebraic Geometry (Cambridge University Press, Cambridge, 2012), A modern view.

[32] I. Dolgachev and J. Keum, 'Birational automorphisms of quartic Hessian surfaces', Trans. Amer. Math. Soc. 354(8) (2002), 3031-3057.

[33] D. Eppstein, 'The many faces of the Nauru graph', http://11011110.livejournal.com/124705 .html.

[34] D. Festi, A. Garbagnati, B. van Geemen and R. van Luijk, 'The Cayley-Oguiso automorphism of positive entropy on a K3 surface', J. Mod. Dyn. 7(1) (2013), 75-97.

[35] I. M. Gelfand, M. M. Kapranov and A. V. Zelevinsky, Discriminants, Resultants and Multidimensional Determinants, Modern Birkhäuser Classics (Birkhäuser Boston Inc., Boston, MA, 2008), Reprint of the 1994 edition. 
[36] E. Ghate and E. Hironaka, 'The arithmetic and geometry of Salem numbers', Bull. Amer. Math. Soc. (N.S.) 38(3) (2001), 293-314.

[37] F. Greer, Z. Li and Z. Tian, 'Picard groups on moduli of K3 surfaces with Mukai models', Int. Math. Res. Not. IMRN (16) (2015), 7238-7257.

[38] J. I. Hutchinson, 'The Hessian of the cubic surface. II', Bull. Amer. Math. Soc. (N.S.) 6(8) (1900), 328-337.

[39] J. I. Hutchinson, 'The Hessian of the cubic surface', Bull. Amer. Math. Soc. (N.S.) 5(6) (1899), 282-292.

[40] D. Huybrechts, 'Lectures on K3 surfaces', http://www.math.uni-bonn.de/people/huybrech/ K3.html.

[41] C. H. Jessop, Quartic Surfaces with Singular Points (Cambridge University Press, Cambridge, 1916).

[42] P. I. Katsylo, 'Rationality of fields of invariants of reducible representations of the group $\mathrm{SL}_{2}$ ', Vestnik Moskov. Univ. Ser. I. Mat. Mekh. (5) (1984), 77-79.

[43] K. Kodaira, 'On compact complex analytic surfaces I', Ann. of Math. (2) 71 (1960), 111-152.

[44] K. Kodaira, 'On compact analytic surfaces. II, III', Ann. of Math. (2) 77 (1963), 563-626. ibid. 78 (1963), 1-40.

[45] S. Kudla, 'A note about special cycles on moduli spaces of K3 surfaces', in Arithmetic and Geometry of K3 Surfaces and Calabi-Yau Threefolds, Fields Institute Communications, 67 (Springer, New York, 2013), 411-427.

[46] G. Lombardo, C. Peters and M. Schuett, 'Abelian fourfolds of Weil type and certain K3 double planes', Preprint, 2012, http://arxiv.org/abs/1209.5997.

[47] S. Ma, 'Rationality of fields of invariants for some representations of $\mathrm{SL}_{2} \times \mathrm{SL}_{2}$ ', Compositio Math. 149(7) (2013), 1225-1234.

[48] K. Matsumoto, T. Sasaki and M. Yoshida, 'The monodromy of the period map of a 4parameter family of $K 3$ surfaces and the hypergeometric function of type $(3,6)$ ', Internat. $J$. Math. 3(1) (1992), 1-164.

[49] D. Maulik and R. Pandharipande, 'Gromov-Witten theory and Noether-Lefschetz theory', in A Celebration of Algebraic Geometry, Clay Mathematics Proceedings, 18 (American Mathematical Society, Providence, RI, 2013), 469-507.

[50] A. L. Mayer, 'Families of $K-3$ surfaces', Nagoya Math. J. 48 (1972), 1-17.

[51] C. T. McMullen, 'Dynamics on $K 3$ surfaces: Salem numbers and Siegel disks', J. Reine Angew. Math. 545 (2002), 201-233.

[52] C. T. McMullen, 'K3 surfaces, entropy and glue', J. Reine Angew. Math. 658 (2011), 1-25.

[53] S. Mukai, 'Curves, $K 3$ surfaces and Fano 3-folds of genus $\leqslant 10$ ', in Algebraic Geometry and Commutative Algebra, I (Kinokuniya, Tokyo, 1988), 357-377.

[54] S. Mukai, 'Polarized K3 surfaces of genus 18 and 20', in Complex Projective Geometry (Trieste, 1989/Bergen, 1989), London Mathematical Society Lecture Note Series, 179 (Cambridge University Press, Cambridge, 1992), 264-276.

[55] S. Mukai, 'Polarized $K 3$ surfaces of genus thirteen', in Moduli Spaces and Arithmetic Geometry, Advanced Studies in Pure Mathematics, 45 (Mathematical Society of Japan, Tokyo, 2006), 315-326.

[56] K. O. Ng, 'The classification of (3, 3, 4) trilinear forms', J. Korean Math. Soc. 39(6) (2002), 821-879.

[57] V. V. Nikulin, 'Kummer surfaces', Izv. Akad. Nauk SSSR Ser. Mat. 39(2) (1975), 278-293, 471. English translation: Math. USSR. Izv. 9(2) (1975), 261-275. 
[58] V. V. Nikulin, 'Finite groups of automorphisms of Kählerian K3 surfaces', Tr. Mosk. Mat. Obs. 38 (1979), 75-137. English translation: Trans. Moscow Math. Soc. 38 (1980), 71-135.

[59] V. V. Nikulin, 'Integer symmetric bilinear forms and some of their geometric applications', Izv. Akad. Nauk SSSR Ser. Mat. 43(1) (1979), 111-177, 238.

[60] Keiji Oguiso, 'The third smallest Salem number in automorphisms of $K 3$ surfaces', in Algebraic Geometry in East Asia-Seoul 2008, Advanced Studies in Pure Mathematics, 60 (Mathematical Society of Japan, Tokyo, 2010), 331-360.

[61] Keiji Oguiso, 'Free automorphisms of positive entropy on smooth Kähler surfaces', Preprint, 2012, http://arxiv.org/abs/arXiv:1202.2637v3.

[62] B. Poonen and M. Stoll, 'Most odd degree hyperelliptic curves have only one rational point', Ann. of Math. (2) 180(3) (2014), 1137-1166.

[63] P. Reschke, 'Salem numbers and automorphisms of complex surfaces', Math. Res. Lett. 19(2) (2012), 475-482.

[64] I. Rivin, 'Algorithm to compute the integral orthogonal group, MathOverflow', http://matho verflow.net/q/143543 (version: 2013-09-29).

[65] T. G. Room, 'Self-transformations of determinantal quartic surfaces. I', Proc. Lond. Math. Soc. (2) 51 (1950), 348-361.

[66] B. Saint-Donat, 'Projective models of $K-3$ surfaces', Amer. J. Math. 96 (1974), 602-639.

[67] A. Shankar and X. Wang, 'Average size of the 2-Selmer group of Jacobians of monic even hyperelliptic curves', Preprint, 2013, http://arxiv.org/abs/1307.3531.

[68] V. Snyder and F. R. Sharpe, 'Certain quartic surfaces belonging to infinite discontinuous Cremonian groups', Trans. Amer. Math. Soc. 16(1) (1915), 62-70.

[69] T. Taniguchi and F. Thorne, 'Secondary terms in counting functions for cubic fields', Duke Math. J. 162(13) (2013), 2451-2508.

[70] J. Thorne, 'The arithmetic of simple singularities', PhD Thesis, Harvard University, 2012.

[71] A. N. Tjurin, 'The intersection of quadrics', Uspekhi Mat. Nauk 30(6(186)) (1975), 51-99.

[72] C. T. C. Wall, 'Pencils of binary quartics', Rend. Semin. Mat. Univ. Padova 99 (1998), 197-217.

[73] K. Wilson, 'Three perspectives on $n$ points in $\mathbb{P}^{n-2}$, , PhD Thesis, Princeton University, 2012.

[74] M. M. Wood, 'Rings and ideals parameterized by binary $n$-ic forms', J. Lond. Math. Soc. (2) 83(1) (2011), 208-231.

[75] M. M. Wood, 'Parametrization of ideal classes in rings associated to binary forms', J. Reine Angew. Math. 689 (2014), 169-199.

[76] D. J. Wright and A. Yukie, 'Prehomogeneous vector spaces and field extensions', Invent. Math. 110(2) (1992), 283-314. 